\title{
Curve counting on abelian surfaces and threefolds
}

\author{
Jim Bryan, Georg Oberdieck, Rahul Pandharipande and Qizheng Yin
}

\begin{abstract}
We study the enumerative geometry of algebraic curves on abelian surfaces and threefolds. In the abelian surface case, the theory is parallel to the well-developed study of the reduced Gromov-Witten theory of $K 3$ surfaces. We prove complete results in all genera for primitive classes. The generating series are quasi-modular forms of pure weight. Conjectures for imprimitive classes are presented. In genus 2 , the counts in all classes are proven. Special counts match the Euler characteristic calculations of the moduli spaces of stable pairs on abelian surfaces by Göttsche-Shende. A formula for hyperelliptic curve counting in terms of Jacobi forms is proven (modulo a transversality statement).

For abelian threefolds, complete conjectures in terms of Jacobi forms for the generating series of curve counts in primitive classes are presented. The base cases make connections to classical lattice counts of Debarre, Göttsche, and Lange-Sernesi. Further evidence is provided by Donaldson-Thomas partition function computations for abelian threefolds. A multiple cover structure is presented. The abelian threefold conjectures open a new direction in the subject.
\end{abstract}

\section{Introduction}

\subsection{Vanishings}

Let $A$ be a complex abelian variety of dimension $d$. The Gromov-Witten invariants of $A$ in genus $g$ and class $\beta \in H_{2}(A, \mathbb{Z})$ are defined by integration against the virtual class of the moduli space of stable maps $\bar{M}_{g, n}(A, \beta)$,

$$
\left\langle\tau_{a_{1}}\left(\gamma_{1}\right) \cdots \tau_{a_{n}}\left(\gamma_{n}\right)\right\rangle_{g, \beta}^{A}=\int_{\left[\bar{M}_{g, n}(A, \beta)\right]^{\mathrm{vir}}} \operatorname{ev}_{1}^{*}\left(\gamma_{1}\right) \psi_{1}^{a_{1}} \cdots \mathrm{ev}_{n}^{*}\left(\gamma_{n}\right) \psi_{n}^{a_{n}} ;
$$

see [PT14] for an introduction. (Here, the domain of a stable map is always taken here to be connected.) However, for abelian varieties of dimension $d \geqslant 2$, the Gromov-Witten invariants often vanish for two independent reasons. Fortunately, both can be controlled. The result is a meaningful and non-trivial enumerative geometry of curves in $A$.

Received 21 November 2015, accepted in final form 5 April 2017.

2010 Mathematics Subject Classification 14N35, 14K99.

Keywords: Gromov-Witten theory, abelian varieties, Donaldson-Thomas theory.

This journal is (C) Foundation Compositio Mathematica 2018. This article is distributed with Open Access under the terms of the Creative Commons Attribution Non-Commercial License, which permits non-commercial reuse, distribution, and reproduction in any medium, provided that the original work is properly cited. For commercial re-use, please contact the Foundation Compositio Mathematica.

J.B. was partially supported by NSERC Accelerator and Discovery grants. The research here was partially carried out during a visit of J.B. to the Forschungsinstitut für Mathematik at ETH Zürich in November 2014. G.O. was supported by the grant SNF-200021-143274. R.P. was partially supported by SNF-200021-143274, ERC2012-AdG-320368-MCSK, SwissMap, and the Einstein Stiftung. Q.Y. was supported by the grant ERC-2012-AdG320368-MCSK. 


\section{CURVE COUNTING ON ABELIAN SURFACES AND THREEFOLDS}

The first source of vanishing is the obstruction theory of stable maps. For dimensions $d \geqslant 2$, the cohomology $H^{2,0}(A, \mathbb{C})=H^{0}\left(A, \Omega_{A}^{2}\right)$ does not vanish and yields a trivial quotient of the obstruction sheaf. As a consequence, the virtual class vanishes [KL13] for non-zero classes $\beta$.

An alternative view of the first vanishing can be obtained by deformation invariance. A homology class $\beta \in H_{2}(A, \mathbb{Z})$ is a curve class if $\beta$ is represented by an algebraic curve on $A$. The Gromov-Witten invariants vanish if $\beta$ is not a curve class since then the moduli space $\bar{M}_{g, n}(A, \beta)$ is empty. After generic deformation of $A$, every non-zero curve class $\beta$ acquires a part of $H^{2,0}(A, \mathbb{C})^{\vee}$ and is no longer the class of an algebraic curve. By deformation invariance, the Gromov-Witten invariants of $A$ then necessarily vanish for all non-zero $\beta$.

Second, an independent source of vanishing arises from the action of the abelian variety $A$ on the moduli space $\bar{M}_{g, n}(A, \beta)$ by translation: most stable maps $f: C \rightarrow A$ appear in $d$ dimensional families. Integrands which are translation invariant almost always lead to vanishing Gromov-Witten invariants. We must therefore impose a $d$-dimensional condition on the moduli space which picks out a single or finite number of curves in each translation class.

Curve classes on $A$ are equivalent to divisor classes on the dual abelian variety $\widehat{A}$. Every curve class $\beta \in H_{2}(A, \mathbb{Z})$ has a type $\left(d_{1}, \ldots, d_{\operatorname{dim} A}\right), \quad d_{i} \geqslant 0$, obtained from the standard divisor theory of $\widehat{A}$. (If $A=E_{1} \times \cdots \times E_{d}$ is the product of elliptic curves $E_{i}$, the class $\beta=\sum_{i} d_{i}\left[E_{i}\right]$ has type $\left(d_{1}, \ldots, d_{\operatorname{dim} A}\right)$. See Section 2.2 for a full discussion.) A curve class $\beta$ is non-degenerate if $d_{i}>0$ for all $i$. Otherwise, $\beta$ is a degenerate curve class. The degenerate case is studied by reducing the dimension of $A$. (A detailed discussion of the degenerate case is given in Section 2.5. In the introduction, we focus on the non-degenerate case.)

Various techniques have been developed in recent years to address the first vanishing. The result in the non-degenerate case is a reduced virtual class $\left[\bar{M}_{g, n}(A, \beta)\right]^{\text {red }}$ with dimension increased by $h^{2,0}(A)$. Integrals against the reduced class are invariant under deformations of $A$ for which $\beta$ stays algebraic. Up to translation, we expect the family of genus $g$ curves in class $\beta$ to be of dimension

$$
\operatorname{vdim} \bar{M}_{g}(A, \beta)+h^{2,0}(A)-d=(d-3)(1-g)+\frac{d(d-1)}{2}-d=(d-3)\left(\frac{d}{2}+1-g\right) .
$$

Hence, for abelian varieties of dimension 1, 2, or 3, we expect families (modulo translation) of dimension $2 g-3, g-2$, or 0 , respectively.

For abelian varieties of dimension $d \geqslant 4$, the reduced virtual dimension (1.1) is non-negative only if

$$
g \leqslant \frac{d}{2}+1 \leqslant d-1
$$

In the non-degenerate case, a generic abelian variety $A$ admits no proper abelian subvariety and thus admits no map from a curve of genus less than $d$. Hence, all invariants vanish.

The Gromov-Witten theory of elliptic curves has been completely solved by Okounkov and Pandharipande in [OP06a, OP06b]. Some special results are known about abelian surfaces [BL99, Deb99, Göt98, Ros14]. We put forth here several results and conjectures concerning the complete Gromov-Witten theory of abelian surfaces and threefolds.

\subsection{Abelian surfaces}

1.2.1 Basic curve counting. Let $A$ be an abelian surface, and let $\beta \in H_{2}(A, \mathbb{Z})$ be a curve class of type $\left(d_{1}, d_{2}\right)$ with $d_{1}, d_{2}>0$. The moduli space

$$
\bar{M}_{g, n}(A, \beta)^{\mathrm{FLS}} \subset \bar{M}_{g, n}(A, \beta)
$$




\section{J. Bryan, G. Oberdieck, R. Pandharipande And Q. Yin}

is the closed substack of $\bar{M}_{g, n}(A, \beta)$ parameterizing maps with image in a fixed linear system (FLS) on $A$. Given a curve $C$ in class $\beta$, the FLS condition naturally picks out $\left(d_{1} d_{2}\right)^{2}$ elements in the translation class of $C$. The FLS moduli space carries a reduced virtual fundamental class [KT14, MP13, STV15]

$$
\left[\bar{M}_{g}(A, \beta)^{\mathrm{FLS}}\right]^{\mathrm{red}}
$$

of virtual dimension $g-2$.

Define $\lambda_{k}$ to be the Chern class $\lambda_{k}=c_{k}(\mathbb{E})$ of the Hodge bundle $\mathbb{E} \rightarrow \bar{M}_{g, n}(A, \beta)$ with fiber $H^{0}\left(C, \omega_{C}\right)$ over the moduli point $[f: C \rightarrow A] \in \bar{M}_{g, n}(A, \beta)$.

There are no genus 0 or 1 curves on a general abelian surface $A$. The most basic genus $g \geqslant 2$ Gromov-Witten invariants of $A$ are

$$
\mathrm{N}_{g, \beta}^{\mathrm{FLS}}=\int_{\left[\bar{M}_{g}(A, \beta)^{\mathrm{FLS}}\right]^{\mathrm{red}}}(-1)^{g-2} \lambda_{g-2} .
$$

The integrand $(-1)^{g-2} \lambda_{g-2}$ corresponds to the natural deformation theory of curves in $A$ when considered inside a Calabi-Yau threefold. The invariants (1.2) are therefore precisely the analogs of the genus $g-2$ Gromov-Witten invariants of $K 3$ surfaces which appear in the Katz-KlemmVafa formula; see [MP13, MPT10, PT16].

By deformation invariance, $\mathrm{N}_{g, \beta}^{\mathrm{FLS}}$ depends only on the type $\left(d_{1}, d_{2}\right)$ of $\beta$. We write

$$
\mathrm{N}_{g, \beta}^{\mathrm{FLS}}=\mathrm{N}_{g,\left(d_{1}, d_{2}\right)}^{\mathrm{FLS}} .
$$

We have the following fully explicit conjecture for these counts.

Conjecture A. For all $g \geqslant 2$ and $d_{1}, d_{2}>0$,

$$
\mathrm{N}_{g,\left(d_{1}, d_{2}\right)}^{\mathrm{FLS}}=\left(d_{1} d_{2}\right)^{2} \frac{2(-1)^{g-2}}{(2 g-2) !} \sum_{k \mid \operatorname{gcd}\left(d_{1}, d_{2}\right)} \sum_{m \mid d_{1} d_{2} / k^{2}} k^{2 g-1} m^{2 g-3} .
$$

The right-hand side incorporates a multiple cover rule which expresses the invariants in imprimitive classes in terms of primitive invariants. ${ }^{1}$ The multiple cover structure is discussed in Section 3.3.

Theorem 1.1. Conjecture $A$ is true in the following cases:

(i) for all $g$ in case $\beta$ is primitive,

(ii) for all $\beta$ in case $g=2$.

For part (i) concerning primitive $\beta$, our method relies on a degeneration formula for GromovWitten invariants of abelian surfaces and calculations in [MPT10]. Via a version of the GromovWitten/Pairs correspondence [PT09], the primitive case also yields an independent proof of the Euler characteristic calculations of relative Hilbert schemes of points by Göttsche and Shende [GS15].

For part (ii) concerning genus 2, the proof is reduced by a method of Debarre [Deb99], Göttsche [Göt98], and Lange-Sernesi [LS02a] to a lattice count in abelian groups. Our results reveal a new and surprising multiple cover structure in these counts.

Conjecture A is parallel to the full Katz-Klemm-Vafa (KKV) conjecture for $K 3$ surfaces. Part (i) of Theorem 1.1 is parallel to the primitive KKV conjecture proven in [MPT10]. Part (ii) is

${ }^{1}$ The class is primitive if and only if $\operatorname{gcd}\left(d_{1}, d_{2}\right)=1$ (or, equivalently, the class can be deformed to type $(1, d)$ ); see Section 2.2. 


\section{CURVE COUNTING ON ABELIAN SURFACES AND THREEFOLDS}

parallel to the full Yau-Zaslow conjecture for rational curves on $K 3$ surfaces proven in [KMPS10]. While our proof of part (i) involves methods parallel to those appearing in the proof of the primitive KKV conjecture, our proof of part (ii) is completely unrelated to the (much more complicated) geometry used in the proof of the full Yau-Zaslow conjecture.

The full Katz-Klemm-Vafa conjecture is proven in [PT16]. However, most cases of Conjecture A remain open.

1.2.2 Point insertions for primitive classes. Let $\mathrm{p} \in H^{4}(A, \mathbb{Z})$ be the class of a point. Define the $\lambda$-twisted Gromov-Witten invariants with $k$ point insertions by

$$
\mathrm{N}_{g, k,\left(d_{1}, d_{2}\right)}^{\mathrm{FLS}}=\int_{\left[\bar{M}_{g, k}(A, \beta)^{\mathrm{FLS}}\right]_{\mathrm{red}}}(-1)^{g-2-k} \lambda_{g-2-k} \prod_{i=1}^{k} \mathrm{ev}_{i}^{*}(\mathrm{p}),
$$

where $\beta$ is a curve class of type $\left(d_{1}, d_{2}\right)$. Define the function

$$
\mathrm{S}(z, \tau)=-\sum_{d \geqslant 1} \sum_{m \mid d} \frac{d}{m}\left(p^{m}-2+p^{-m}\right) q^{d},
$$

considered as a formal power series in the variables $p=e^{2 \pi i z}$ and $q=e^{2 \pi i \tau}$.

Theorem 1.2. After setting $u=2 \pi z$, we have

$$
\sum_{g \geqslant 2} \sum_{d \geqslant 1} \mathrm{~N}_{g, k,(1, d)}^{\mathrm{FLS}} u^{2 g-2} q^{d}=q \frac{d}{d q}\left(\frac{\mathrm{S}(z, \tau)^{k+1}}{k+1}\right) .
$$

For $k=0$, by definition, $\mathrm{N}_{g, 0,(1, d)}^{\mathrm{FLS}}=\mathrm{N}_{g,(1, d)}^{\mathrm{FLS}}$. Hence, by Theorem 1.2,

$$
\sum_{g \geqslant 2} \sum_{d \geqslant 1} \mathrm{~N}_{g,(1, d)}^{\mathrm{FLS}} u^{2 g-2} q^{d}=q \frac{d}{d q} \mathrm{~S}(z, \tau)
$$

which is a restatement of the formula of Conjecture A for the classes $(1, d)$. Theorem 1.2 specializes in the $k=0$ case to the primitive part of Theorem 1.1.

1.2.3 Quasi-modular forms. Let $\gamma_{1}, \ldots, \gamma_{n} \in H^{*}(A, \mathbb{Q})$ be cohomology classes. The primitive descendent potential of $A$ with insertions $\tau_{a_{1}}\left(\gamma_{1}\right), \ldots, \tau_{a_{n}}\left(\gamma_{n}\right)$ is defined ${ }^{2}$ by

$$
\mathrm{F}_{g}^{A}\left(\tau_{a_{1}}\left(\gamma_{1}\right) \cdots \tau_{a_{n}}\left(\gamma_{n}\right)\right)=\sum_{d \geqslant 0}\left\langle\tau_{a_{1}}\left(\gamma_{1}\right) \cdots \tau_{a_{n}}\left(\gamma_{n}\right)\right\rangle_{g,(1, d)}^{A, \text { red }} q^{d}
$$

where the coefficients on the right-hand side denote the reduced invariants of $A$ :

$$
\left\langle\tau_{a_{1}}\left(\gamma_{1}\right) \cdots \tau_{a_{n}}\left(\gamma_{n}\right)\right\rangle_{g, \beta}^{A, \mathrm{red}}=\int_{\left[\bar{M}_{g, n}(A, \beta)\right]^{\mathrm{red}}} \prod_{i=1}^{n} \operatorname{ev}_{i}^{*}\left(\gamma_{i}\right) \psi_{i}^{a_{i}} .
$$

The ring QMod of holomorphic quasi-modular forms (of level 1) is the free polynomial algebra in the Eisenstein series $E_{2}(\tau), E_{4}(\tau)$, and $E_{6}(\tau)$ :

$$
\mathrm{QMod}=\mathbb{Q}\left[E_{2}, E_{4}, E_{6}\right] .
$$

\footnotetext{
${ }^{2}$ Unlike in the FLS setting, the degenerate type $(1,0)$ is included here.
} 


\section{J. Bryan, G. Oberdieck, R. Pandharipande And Q. Yin}

(The Eisenstein series are defined by $E_{2 k}(\tau)=1-\left(4 k / B_{2 k}\right) \sum_{m \mid d} m^{2 k-1} q^{d}$, where $B_{2 k}$ is the Bernoulli number.) The ring QMod carries a grading by weight,

$$
\mathrm{QMod}=\bigoplus_{k \geqslant 0} \mathrm{QMod}_{2 k}
$$

where $E_{2 k}$ has weight $2 k$. Let

$$
\mathrm{QMod}_{\leqslant 2 k} \subset \text { QMod }
$$

be the linear subspace of quasi-modular forms of weight at most $2 k$.

The series vanish in $g=0$. For $g \geqslant 1$ and arbitrary insertions, we have the following result.

Theorem 1.3. The series $\mathrm{F}_{g}^{A}\left(\tau_{a_{1}}\left(\gamma_{1}\right) \cdots \tau_{a_{n}}\left(\gamma_{n}\right)\right)$ is the Fourier expansion in $q=e^{2 \pi i \tau}$ of a quasimodular form of weight at most $2(g-2)+2 n$,

$$
\mathrm{F}_{g}^{A}\left(\tau_{a_{1}}\left(\gamma_{1}\right) \cdots \tau_{a_{n}}\left(\gamma_{n}\right)\right) \in \mathrm{QMod}_{\leqslant 2(g-2)+2 n} .
$$

A sharper formulation of Theorem 1.3 specifying the weight appears in Theorem 5.1.

1.2.4 Hyperelliptic curves. A non-singular curve $C$ of genus $g \geqslant 2$ is hyperelliptic if $C$ admits a degree 2 map $C \rightarrow \mathbb{P}^{1}$. A stable curve $C$ is hyperelliptic if $[C] \in \bar{M}_{g}$ is in the closure of the locus of non-singular hyperelliptic curves. (The closure can be described precisely via the theory of admissible covers [HM82].) An irreducible hyperelliptic curve $C$ of genus $g$ on an abelian surface $A$ is the image of a stable map $f: \widehat{C} \rightarrow C \subset A$ satisfying the following two conditions:

- $\widehat{C}$ is an irreducible stable hyperelliptic curve of genus $g$;

- $f: \widehat{C} \rightarrow C$ is birational.

By [Pir89], for any abelian surface $A$ and curve class $\beta$, the number of irreducible hyperelliptic curves of genus $g$ in a fixed linear system of class $\beta$ is finite. ${ }^{3}$ We write $\mathrm{h}_{g, \beta}^{A, F L S}$ for this finite count. Unlike all other invariants considered in the paper, $\mathrm{h}_{g, \beta}^{A, \mathrm{FLS}}$ is defined by classical counting.

Since every genus 2 curve is hyperelliptic, for generic $A$ and $\beta$ of type $(1, d)$, we have

$$
\mathrm{h}_{2, \beta}^{A, F L S}=d^{2} \sum_{m \mid d} m
$$

by the genus 2 part of Theorem 1.1. The following result calculates the genus 3 hyperelliptic counts in generic primitive classes.

Proposition 1.4. For a generic abelian surface $A$ with a curve class $\beta$ of type $(1, d)$,

$$
\mathrm{h}_{3, \beta}^{A, \mathrm{FLS}}=d^{2} \sum_{m \mid d} \frac{m\left(3 m^{2}+1-4 d\right)}{4} .
$$

Let $\mathcal{H}_{g}$ be the stack fundamental class of the closure of non-singular hyperelliptic curves inside $\bar{M}_{g}$. By [FP05], the class $\mathcal{H}_{g}$ is tautological of codimension $g-2$ (see [FP13] for an introduction to tautological classes on the moduli spaces of curves). While the restriction of $\mathcal{H}_{g}$ to $M_{g}$ is a known multiple of $\lambda_{g-2}$, a closed formula for $\mathcal{H}_{g}$ on $\bar{M}_{g}$ in terms of the standard generators of the tautological ring is not known.

\footnotetext{
${ }^{3}$ On the other hand, generic abelian varieties of dimension at least 3 contain no hyperelliptic curve at all; see [Pir89].
} 


\section{Curve COUnting on ABElian SURFACES AND THREefoldS}

For $\beta$ of type $\left(d_{1}, d_{2}\right)$, we define a virtual count of hyperelliptic curves in class $\beta$ by

$$
\mathrm{H}_{g,\left(d_{1}, d_{2}\right)}^{\mathrm{FLS}}=\int_{\left[\bar{M}_{g}(A, \beta)^{\mathrm{FLS}}\right]_{\mathrm{red}}} \pi^{*}\left(\mathcal{H}_{g}\right),
$$

where $\pi$ is the forgetful map $\pi: \bar{M}_{g}(A, \beta) \rightarrow \bar{M}_{g}$. Because the integral (1.3) is deformation invariant, the left side depends only upon $g$ and $\left(d_{1}, d_{2}\right)$.

For irreducible curve classes $\beta$ of type $(1, d)$ on an abelian surface $A$, consider the following property:

(†) Every irreducible curve in $\mathbb{P}^{1} \times A$ of class

$$
(2, \beta)=2\left[\mathbb{P}^{1}\right]+\beta \in H_{2}\left(\mathbb{P}^{1} \times A, \mathbb{Z}\right)
$$

is non-singular.

We will prove property $(\dagger)$ for curves of genus 2 in case $A$ and $\beta$ are generic. Together with the explicit expression [HM82] for $\mathcal{H}_{3} \in H^{2}\left(\bar{M}_{3}, \mathbb{Q}\right)$, this implies Proposition 1.4. The existence of classes $\beta$ of type $(1, d)$ satisfying $(\dagger)$ is not known for most $d$, but is expected generically for dimension reasons.

Define the Jacobi theta function [Cha85]

$$
K(z, \tau)=\frac{i \vartheta_{1}(z, \tau)}{\eta(\tau)^{3}}=i u \exp \left(\sum_{k \geqslant 1} \frac{(-1)^{k} B_{2 k}}{2 k(2 k) !} E_{2 k}(\tau) u^{2 k}\right),
$$

where $u=2 \pi z$.

Theorem 1.5. Let $\beta$ be an irreducible class of type $(1, d)$ on an abelian surface $A$ satisfying $(\dagger)$. Then the following hold:

(i) $\sum_{g \geqslant 2} \mathrm{~h}_{g, \beta}^{A, \mathrm{FLS}}(2 \sin (u / 2))^{2 g+2}=\sum_{g \geqslant 2} \mathrm{H}_{g,(1, d)}^{\mathrm{FLS}} u^{2 g+2}$.

(ii) After the change of variables $u=2 \pi z$ and $q=e^{2 \pi i \tau}$, we have

$$
\sum_{g \geqslant 2} \mathrm{H}_{g,(1, d)}^{\mathrm{FLS}} u^{2 g+2}=\operatorname{Coeff}_{q^{d}}\left[\left(q \frac{d}{d q}\right)^{2} \frac{K(z, \tau)^{4}}{4}\right],
$$

where Coeff $q^{d}$ denotes the coefficient of $q^{d}$.

Enumerative results on hyperelliptic curves via Gromov-Witten theory were first obtained for $\mathbb{P}^{2}$ by Graber [Gra01] using the Hilbert scheme of points $\operatorname{Hilb}^{2}\left(\mathbb{P}^{2}\right)$. The hyperelliptic curve counts on abelian surfaces were first studied by Rose [Ros14] using the orbifold Gromov-Witten theory of $\operatorname{Sym}^{2}(A)$ and the geometry of the Kummer surface. Rose derives his results from the crepant resolution conjecture (CRC) [BG09, Rua06] and certain geometric genericity assumptions. While our approach is similar, the closed formula (ii) for $\mathrm{H}_{g,(1, d)}^{\mathrm{FLS}}$ via the theta function $K(z, \tau)$ is new.

Proposition 1.4 for $\mathrm{h}_{3, \beta}^{A, \mathrm{FLS}}$ and the formula of Theorem 1.5 for $\mathrm{h}_{g, \beta}^{A, \mathrm{FLS}}$ in higher genus (obtained by combining parts (i) and (ii)) do not match Rose's results. The errors in Rose's genus 3 counts can be repaired to agree with Proposition 1.4. We hope that the CRC approach will be able to arrive exactly at the formula of Theorem 1.5 for $\mathrm{h}_{g, \beta}^{A, F L S}$.

The values of $\mathrm{h}_{g, \beta}^{A, \mathrm{FLS}}$ in low genus and degree are presented in Table 1 . The distribution of the non-zero values in Table 1 matches precisely the results of Knutsen, Lelli-Chiesa, and Mongardi 
J. Bryan, G. Oberdieck, R. Pandharipande and Q. Yin

\begin{tabular}{|c|c|c|c|c|c|c|c|c|c|c|}
\hline$g d$ & 1 & 2 & 3 & 4 & 5 & 6 & 7 & 8 & 9 & 10 \\
\hline 2 & 1 & 12 & 36 & 112 & 150 & 432 & 392 & 960 & 1053 & 1800 \\
3 & 0 & 6 & 90 & 456 & 1650 & 4320 & 9996 & 20640 & 36774 & 67500 \\
4 & 0 & 0 & 9 & 192 & 1425 & 6732 & 23814 & 68352 & 173907 & 387900 \\
5 & 0 & 0 & 0 & 4 & 150 & 1656 & 10486 & 48240 & 174474 & 539200 \\
6 & 0 & 0 & 0 & 0 & 0 & 36 & 735 & 6720 & 41310 & 191400 \\
7 & 0 & 0 & 0 & 0 & 0 & 0 & 0 & 96 & 1620 & 14700 \\
8 & 0 & 0 & 0 & 0 & 0 & 0 & 0 & 0 & 0 & 100 \\
\hline
\end{tabular}

TABLE 1 . The first values for the counts $\mathrm{h}_{g, \beta}^{A, F L S}$ of hyperelliptic curves of genus $g$ and type $(1, d)$ in a FLS of a generic abelian surface $A$ as predicted by Theorem 1.5

in [KLCM17, Theorem 1.6]: $\mathrm{h}_{g, \beta}^{A, F L S}$ is non-zero if and only if

$$
(g-1)+\left\lfloor\frac{g-1}{4}\right\rfloor\left((g-1)-2\left\lfloor\frac{g-1}{4}\right\rfloor-2\right) \leqslant d .
$$

The entries for $(g, d)=(4,3)$ and $(g, d)=(5,4)$ have recently been confirmed in [BS17, BO17].

\subsection{Abelian threefolds}

1.3.1 Donaldson-Thomas theory. Let $X$ be an abelian threefold, and let $\beta \in H_{2}(X, \mathbb{Z})$ be a curve class. The Hilbert scheme of curves

$$
\operatorname{Hilb}^{n}(X, \beta)=\left\{Z \subset X \mid[Z]=\beta, \chi\left(\mathcal{O}_{Z}\right)=n\right\}
$$

parameterizes 1-dimensional subschemes of class $\beta$ with holomorphic Euler characteristic $n$. The group $X$ acts on $\operatorname{Hilb}^{n}(X, \beta)$ by translation.

If $n \neq 0$, no assumption on $\beta$ is made. If $n=0$, we assume that $\beta$ is not of type $(d, 0,0)$ up to permutation. Then, the action of $X$ has finite stabilizers and the stack quotient $\operatorname{Hilb}^{n}(X, \beta) / X$ is a Deligne-Mumford stack.

We consider here two numerical invariants of $\operatorname{Hilb}^{n}(X, \beta) / X$, the topological Euler characteristic

$$
\widehat{\mathrm{DT}}_{n, \beta}^{X}=e\left(\operatorname{Hilb}^{n}(X, \beta) / X\right)
$$

and the reduced Donaldson-Thomas invariant of $X$ defined as the Behrend function weighted Euler characteristic

$$
\mathrm{DT}_{n, \beta}^{X}=e\left(\operatorname{Hilb}^{n}(X, \beta) / X, \nu\right)=\sum_{k \in \mathbb{Z}} k \cdot e\left(\nu^{-1}(k)\right)
$$

While the Behrend function

$$
\nu: \operatorname{Hilb}^{n}(X, \beta) / X \rightarrow \mathbb{Z}
$$

is integer valued, the topological Euler characteristic $e$ is taken in the orbifold sense and so may be a rational number. Hence,

$$
\mathrm{DT}_{n, \beta}^{X} \in \mathbb{Q}, \quad \widehat{\mathrm{DT}}_{n, \beta}^{X} \in \mathbb{Q} .
$$

By results of Gulbrandsen [Gul13], we know that $\mathrm{DT}_{n, \beta}^{X}$ is invariant under deformations of the pair $(X, \beta)$ if $n \neq 0$. For $n=0$, Gulbrandsen's method breaks down, but deformation invariance is still expected. The numbers $\widehat{\mathrm{DT}}_{n, \beta}^{X}$ are not expected to be deformation invariant. 


\section{Curve COUnting on ABElian SURFACES AND THREEFoldS}

By deformation equivalence, we may compute $\mathrm{DT}_{n, \beta}^{X}$ after specialization to the product geometry $X=A \times E$. We compute $\widehat{\mathrm{DT}}_{n, \beta}^{X}$ for the product geometry, and we conjecture a simple relationship there between $\mathrm{DT}_{n, \beta}^{X}$ and $\widehat{\mathrm{DT}}_{n, \beta}^{X}$. We then obtain a formula for $\mathrm{DT}_{n, \beta}^{X}$.

Let $A$ be a generic abelian surface carrying a curve class $\beta_{d^{\prime}}$ of type $\left(1, d^{\prime}\right)$ with $d^{\prime}>0$, and let $E$ be a generic elliptic curve. Consider the abelian threefold $X=A \times E$. The curve class

$$
\left(\beta_{d^{\prime}}, d\right)=\beta_{d^{\prime}}+d[E] \in H_{2}(X, \mathbb{Z})
$$

is of type $\left(1, d^{\prime}, d\right)$.

The following result determines the invariants $\widehat{\mathrm{DT}}_{n,\left(\beta_{d^{\prime}}, d\right)}^{X}$ in the first two non-trivial cases $d^{\prime}=1$ and $d^{\prime}=2$.

Let $K$ be the theta function which already appeared in Section 1.2.4,

$$
K(p, q)=\left(p^{1 / 2}-p^{-1 / 2}\right) \prod_{m \geqslant 1} \frac{\left(1-p q^{m}\right)\left(1-p^{-1} q^{m}\right)}{\left(1-q^{m}\right)^{2}} .
$$

Theorem 1.6. For the topological Euler characteristic theory, we have

(i) $\sum_{d \geqslant 0} \sum_{n \in \mathbb{Z}} \widehat{\mathrm{DT}}_{n,\left(\beta_{1}, d\right)}^{X} p^{n} q^{d}=K(p, q)^{2}$,

(ii) $\sum_{d \geqslant 0} \sum_{n \in \mathbb{Z}} \widehat{\mathrm{DT}}_{n,\left(\beta_{2}, d\right)}^{X} p^{n} q^{d}=K(p, q)^{4} \cdot\left(\frac{1}{2}+\frac{3 p}{(1-p)^{2}}+\sum_{d \geqslant 1} \sum_{k \mid d} k \cdot\left(3\left(p^{k}+p^{-k}\right) q^{d}+12 q^{2 d}\right)\right)$.

Assuming Conjecture D in Section 7.6, we obtain the following result for the invariants $\mathrm{DT}_{n,\left(\beta_{d^{\prime}}, d\right)}^{X}$ in the cases $d^{\prime}=1$ and $d^{\prime}=2$.

Consider the Weierstrass elliptic function

$$
\wp(p, q)=\frac{1}{12}+\frac{p}{(1-p)^{2}}+\sum_{d \geqslant 1} \sum_{m \mid d} m\left(p^{m}-2+p^{-m}\right) q^{d}
$$

expanded in the region $|p|<1$.

Corollary* 1.7. Assume that Conjecture D holds. Then we have

(i) $\sum_{d \geqslant 0} \sum_{n \in \mathbb{Z}} \mathrm{DT}_{n,\left(\beta_{1}, d\right)}^{X}(-p)^{n} q^{d}=-K(p, q)^{2}$,

(ii) $\sum_{d \geqslant 0} \sum_{n \in \mathbb{Z}} \mathrm{DT}_{n,\left(\beta_{2}, d\right)}^{X}(-p)^{n} q^{d}=-\frac{3}{2} K(p, q)^{4} \wp(p, q)-\frac{3}{8} K\left(p^{2}, q^{2}\right)^{2}$.

Part (i) of Corollary* 1.7 verifies an earlier prediction of BPS counts on abelian threefolds by Maldacena, Moore, and Strominger [MMS99].

Theorem 1.6 concerns the Hilbert schemes of curves on $X$. The Euler characteristics associated with the Hilbert scheme of points of $X$ (via the generalized Kummer construction) have been calculated recently by Shen [She15], proving a conjecture of Gulbrandsen [Gul13].

1.3.2 Gromov-Witten theory. Let $X$ be an abelian threefold, and let $\beta$ be a curve class of type $\left(d_{1}, d_{2}, d_{3}\right)$ with $d_{1}, d_{2}>0$. We consider curves of genus $g \geqslant 2$.

The translation action of $X$ on $\bar{M}_{g}(X, \beta)$ has finite stabilizer. Hence, $\bar{M}_{g}(X, \beta) / X$ is a Deligne-Mumford stack. In Section 8, we use methods of Kiem and Li [KL13] to construct a 


\section{J. Bryan, G. Oberdieck, R. Pandharipande and Q. Yin}

reduced virtual class $\left[\bar{M}_{g}(X, \beta) / X\right]^{\text {red }}$ on $\bar{M}_{g}(X, \beta) / X$ of dimension 0 . We define the reduced quotient Gromov-Witten invariants of $X$ by

$$
\mathrm{N}_{g, \beta}=\int_{\left[\bar{M}_{g}(X, \beta) / X\right]^{\mathrm{red}}} 1 .
$$

By construction, $\mathrm{N}_{g, \beta}$ is deformation invariant and hence depends only on $g$ and the type $\left(d_{1}, d_{2}, d_{3}\right)$ of $\beta$. We write

$$
\mathrm{N}_{g, \beta}=\mathrm{N}_{g,\left(d_{1}, d_{2}, d_{3}\right)}
$$

The number $\mathrm{N}_{g, \beta}$ is a virtual count of translation classes of genus $g$ curves of class $\beta$ in $X$. In Section 8, we show that $\mathrm{N}_{g, \beta}$ determines the full reduced descendent Gromov-Witten theory of $X$ in genus $g$ and class $\beta$.

The following conjecture relates the Gromov-Witten invariants $\mathrm{N}_{g, \beta}$ to the DonaldsonThomas invariants $\mathrm{DT}_{n, \beta}^{X}$ defined above. Define the generating series

$$
Z_{\beta}^{\mathrm{GW}}(u)=\sum_{g \geqslant 2} \mathrm{~N}_{g, \beta} u^{2 g-2} \text { and } Z_{\beta}^{\mathrm{DT}}(y)=\sum_{n \in \mathbb{Z}} \mathrm{DT}_{n, \beta}^{X} y^{n} .
$$

Conjecture B. The series $Z_{\beta}^{\mathrm{DT}}(y)$ is the Laurent expansion of a rational function in $y$, and we have

$$
Z_{\beta}^{\mathrm{DT}}(y)=Z_{\beta}^{\mathrm{GW}}(u)
$$

after the variable change $y=-e^{i u}$.

Conjecture B is a Gromov-Witten/Donaldson-Thomas correspondence for reduced theories [MNOP06, OP16]. In conjunction with part (i) of Corollary* 1.7 and the expansion (1.4), Conjecture B determines the invariants $\mathrm{N}_{g,(1,1, d)}$ for all $d \geqslant 0$ by the formula

$$
\begin{aligned}
\sum_{d \geqslant 0} \sum_{g \geqslant 2} \mathrm{~N}_{g,(1,1, d)} u^{2 g-2} q^{d} & =-\left.K^{2}(z, \tau)\right|_{y=-e^{2 \pi i z}, q=e^{2 \pi i \tau}} \\
& =\left(y+2+y^{-1}\right) \prod_{m \geqslant 1} \frac{\left(1+y q^{m}\right)^{2}\left(1+y^{-1} q^{m}\right)^{2}}{\left(1-q^{m}\right)^{4}} .
\end{aligned}
$$

To capture the invariants $\mathrm{N}_{g,\left(1, d^{\prime}, d\right)}$ for higher $d^{\prime}$, we conjecture an additional structure governing the counting. Let

$$
f_{\left(d_{1}, d_{2}, d_{3}\right)}(u)=\sum_{g \geqslant 2} \mathrm{~N}_{g,\left(d_{1}, d_{2}, d_{3}\right)} u^{2 g-2} .
$$

The following multiple cover rule expresses the invariants of type $\left(1, d^{\prime}, d\right)$ in terms of those of type $(1,1, d)$.

Conjecture C. For all $d^{\prime}, d>0$, we have

$$
f_{\left(1, d^{\prime}, d\right)}(u)=\sum_{k \mid \operatorname{gcd}\left(d^{\prime}, d\right)} \frac{1}{k} f_{\left(1,1, d^{\prime} d / k^{2}\right)}(k u) .
$$

Conjecture C matches the counts of genus 3 curves by the lattice method of [Deb99, Göt98, LS02a]. The deepest support for Conjecture C is a highly non-trivial match with part (ii) of Corollary* 1.7. (Recently, further evidence has been obtained in [OS16a].)

Taken together, Corollary* 1.7 and Conjectures $\mathrm{B}$ and $\mathrm{C}$ determine the invariants $\mathrm{N}_{g, \beta}$ for all primitive classes $\beta$ (by definition, the class is primitive if and only if it can be deformed to 


\section{Curve COUnting on ABElian SURFACES AND THREefoldS}

type $\left(1, d^{\prime}, d\right)$; see Section 2.2). The discussion is parallel to [OP16, Conjecture A] concerning the virtual enumeration of curves on $K 3 \times E$ in classes $(\beta, d)$ where $\beta \in H_{2}(K 3, \mathbb{Z})$ is primitive. The latter has been computed for $\langle\beta, \beta\rangle \in\{-2,0\}$ in Donaldson-Thomas theory in [Bry15]. The proof of Theorem 1.6 in Section 7 closely follows the strategy of [Bry15].

For Theorem 1.6 and Conjecture B, the Hilbert scheme of curves can be replaced by the moduli space of stable pairs [PT09]. For technical aspects of the proof of Theorem 1.6, ideal sheaves are simpler, but there is no fundamental difference in the arguments required here.

A multiple cover formula for BPS counts in classes $\left(1, d, d^{\prime}\right)$ was proposed in [MMS99]. However, the formula in [MMS99] is different from ours and does not match the genus 3 counts or Corollary* 1.7.

An extension of Conjecture C to all curve classes is discussed in Section 8.6.

\subsection{Plan of the paper}

In Section 2, we recall several classical facts concerning divisors and curves on abelian varieties. Polarized isogenies, which play a central role in the enumeration of low-genus curves, are reviewed. Reduced virtual classes are discussed in Section 2.4. Degenerate curves classes are analyzed in Section 2.5.

Part I of the paper (Sections 3-6) concerns the enumeration of curves on abelian surfaces $A$. In Section 3, the genus 2 part of Theorem 1.1 is proven. In Section 4, the proofs of Theorems 1.1 and 1.2 for primitive classes are completed. A connection with the Euler characteristic calculations of stable-pairs moduli spaces on $A$ by Göttsche and Shende [GS15] is discussed in Section 4.7. The quasi-modularity of the primitive descendent potentials of $A$ is studied in Section 5, where a refinement of Theorem 1.3 is proven. A parallel refined quasi-modularity result for the reduced Gromov-Witten theory of $K 3$ surfaces is presented in Section 5.6. The enumeration of hyperelliptic curves on $A$ and the proof of Theorem 1.5 are given in Section 6.

Part II of the paper (Sections 7-8) concerns the enumeration of curves on abelian threefolds $X$. In Section 7, the topological and Behrend weighted Euler characteristics of the Hilbert scheme of curves in $A \times E$ are studied. For $d^{\prime} \in\{1,2\}$, the topological Euler characteristic theory is calculated and Theorem 1.6 is proven (except for genus 3 lattice counts, which appear in Section 8.4). Conjecture D relating the two theories is presented in Section 7.6. In Section 8, the foundations of the quotient Gromov-Witten theory are discussed and the full descendent theory

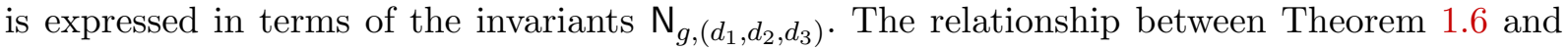
Conjectures $\mathrm{B}$ and $\mathrm{C}$ is studied in Section 8.5. Finally, a multiple cover formula for imprimitive classes is proposed in Section 8.6.

\section{Abelian varieties}

\subsection{Overview}

We review here some basic facts about divisor classes and curve classes on abelian varieties. A standard reference for complex abelian varieties is [BL04]. A treatment of polarized isogenies is required for the lattice counting in Sections 3 and 8.4. Using results of Kiem-Li [KL13], we define reduced virtual classes on the moduli spaces of stable maps to abelian varieties. Finally, we show that the (reduced) Gromov-Witten theory of abelian varieties of arbitrary dimensions is determined by the (reduced) theories in dimensions up to 3. 


\section{J. Bryan, G. Oberdieck, R. Pandharipande And Q. Yin}

\subsection{Curve classes}

Let $V=\mathbb{C}^{n}$. Let $\Lambda \subset V$ be a rank $2 n$ lattice for which $A=V / \Lambda$ is an $n$-dimensional compact complex torus. Let $L$ be a holomorphic line bundle on $A$. The first Chern class $c_{1}(L) \in H^{2}(A, \mathbb{Z})$ induces a Hermitian form $H: V \times V \rightarrow \mathbb{C}$ and an alternating form $E=\operatorname{Im} H: \Lambda \times \Lambda \rightarrow \mathbb{Z}$. By the elementary divisor theorem, there exists a symplectic basis of $\Lambda$ in which $E$ is given by the matrix

$$
\left(\begin{array}{cc}
0 & D \\
-D & 0
\end{array}\right)
$$

where $D=\operatorname{Diag}\left(d_{1}, \ldots, d_{n}\right)$ with integers $d_{i} \geqslant 0$ satisfying $d_{1}\left|d_{2}\right| \cdots \mid d_{n}$. The tuple $\left(d_{1}, \ldots, d_{n}\right)$ is uniquely determined by $L$ (in fact by $c_{1}(L)$ ) and is called the type of $L$.

A polarization on $A$ is a first Chern class $c_{1}(L)$ with positive-definite Hermitian form $H$ (in particular, $\left.d_{i}>0\right)$. The polarization is principal if $d_{i}=1$ for all $i$. The moduli space of polarized $n$-dimensional abelian varieties of a fixed type is irreducible of dimension $n(n+1) / 2$.

Let $\beta \in H_{2}(A, \mathbb{Z})$ be a curve class on $A$. The class corresponds to

$$
\widehat{\beta}=c_{1}(\widehat{L}) \in H^{2}(\widehat{A}, \mathbb{Z}),
$$

where $\widehat{A}=\operatorname{Pic}^{0}(A)$ is the dual complex torus of $A$ and $\widehat{L}$ is a line bundle on $\widehat{A}$. We define the type $\left(d_{1}, \ldots, d_{n}\right)$ of $\beta$ to be the type of $\widehat{L}$. The class $\beta$ is primitive if and only if $d_{1}=1$. For abelian surfaces, we may view $\beta$ as either a curve class or a divisor class: the resulting types are the same.

If $\beta$ is of type $\left(d_{1}, \ldots, d_{n}\right)$ with $d_{i}>0$ for all $i$, then $\widehat{\beta}$ is a polarization on $\widehat{A}$. Hence, all curve classes of a fixed type $\left(d_{1}, \ldots, d_{n}\right)$ with $d_{i}>0$ for all $i$ are deformation equivalent.

If $d_{i}=0$ for some $i$, then we say that $\beta$ is of degenerate type. Write $k=\max \left\{i \mid d_{i} \neq 0\right\}$. By [BL04, Theorem 3.3.3], there exist a subtorus $B \subset \widehat{A}$ of dimension $n-k$ with quotient map

$$
p: \widehat{A} \rightarrow \bar{A}=\widehat{A} / B
$$

and a polarization

$$
\bar{\beta}=c_{1}(\bar{L}) \in H^{2}(\bar{A}, \mathbb{Z})
$$

of type $\left(d_{1}, \ldots, d_{k}\right)$ such that $\widehat{\beta}=p^{*}(\bar{\beta})$. The deformation of $\beta$ is then governed by the deformation of $\bar{\beta}$. As a result, curve classes of a fixed type $\left(d_{1}, \ldots, d_{k}, 0, \ldots, 0\right)$ are also deformation equivalent.

Let $A$ be the product of $n$ elliptic curves $E_{1} \times \cdots \times E_{n}$. For integers $a_{1}, \ldots, a_{n} \geqslant 0$, consider the curve class

$$
\beta=a_{1}\left[E_{1}\right]+\cdots+a_{n}\left[E_{n}\right] \in H_{2}(A, \mathbb{Z}) .
$$

The type $\left(d_{1}, \ldots, d_{n}\right)$ of $\beta$ is given by the rank and the invariant factors of the abelian group associated with $\left(a_{1}, \ldots, a_{n}\right)$ :

$$
\bigoplus_{i=1}^{n} \mathbb{Z} / a_{i} \cong \mathbb{Z}^{m} \oplus \bigoplus_{j=1}^{k} \mathbb{Z} / d_{j}^{\prime}
$$

Here, $k, m \leqslant n$ and

$$
\left(d_{1}, \ldots, d_{n}\right)=(\underbrace{1, \ldots, 1}_{n-k-m}, d_{1}^{\prime}, \ldots, d_{k}^{\prime}, \underbrace{0, \ldots, 0}_{m}) .
$$

Later, we shall also say that $\beta$ is of type $\left(a_{1}, \ldots, a_{n}\right)$ without requiring $a_{1}\left|a_{2}\right| \cdots \mid a_{n}$. 


\section{Curve Counting on ABElian SURFACES AND ThreEFoldS}

Two tuples $\left(a_{1}, \ldots, a_{n}\right)$ and $\left(b_{1}, \ldots, b_{n}\right)$ are deformation equivalent if and only if

$$
\bigoplus_{i=1}^{n} \mathbb{Z} / a_{i} \cong \bigoplus_{i=1}^{n} \mathbb{Z} / b_{i} \text {. }
$$

The primitivity of $\beta$ is determined by $\operatorname{gcd}\left(a_{1}, \ldots, a_{n}\right)$.

\subsection{Polarized isogenies}

The following discussion is based on [Deb99, Göt98, LS02a]. Let $f: C \rightarrow A$ be a map from a non-singular curve of genus $g$. Suppose that the curve class $\beta=f_{*}[C]$ is of type $\left(d_{1}, \ldots, d_{n}\right)$ with $d_{i}>0$ for all $i$. The map $f$ factors as

$$
C \stackrel{\text { aj }}{\rightarrow} J \stackrel{\pi}{\rightarrow} A,
$$

where $J$ is the Jacobian of $C$ and aj is the Abel-Jacobi map, defined up to translation by $J$. By duality, $\pi$ corresponds to

$$
\widehat{\pi}: \widehat{A} \rightarrow J \text { such that } \widehat{\beta}=\widehat{\pi}^{*} \theta,
$$

where $\theta$ is the theta divisor class on $J$ (here, we identify $J$ with $\widehat{J}$ ). When $g=n$, the map $\widehat{\pi}$ is a polarized isogeny.

More generally, consider the isogeny

$$
\phi_{\widehat{\beta}}: \widehat{A} \rightarrow \widehat{\widehat{A}} \cong A, \quad x \mapsto t_{x}^{*} \widehat{L} \otimes \widehat{L}^{-1},
$$

where $\widehat{\beta}=c_{1}(\widehat{L})$ for some line bundle $\widehat{L}$ and $t_{x}: \widehat{A} \rightarrow \widehat{A}$ is the translation by $x$. The finite kernel of $\phi_{\widehat{\beta}}$ admits a non-degenerate multiplicative alternating form

$$
\langle,\rangle: \operatorname{Ker}\left(\phi_{\widehat{\beta}}\right) \times \operatorname{Ker}\left(\phi_{\widehat{\beta}}\right) \rightarrow \mathbb{C}^{*},
$$

called the commutator pairing. By [BL04, Corollary 6.3.5], there is a bijective correspondence between the following two sets:

- polarized isogenies from $(\widehat{A}, \widehat{\beta})$ to principally polarized abelian varieties $(B, \theta)$,

- maximal totally isotropic subgroups of $\operatorname{Ker}\left(\phi_{\widehat{\beta}}\right)$.

The cardinality of both sets depends only on the type $\left(d_{1}, \ldots, d_{n}\right)$ of $\beta$ and is denoted by $\nu\left(d_{1}, \ldots, d_{n}\right)$. In fact, under a suitable basis of $\widehat{\Lambda}$, we have

$$
\operatorname{Ker}\left(\phi_{\widehat{\beta}}\right) \cong\left(\mathbb{Z} / d_{1} \times \cdots \times \mathbb{Z} / d_{n}\right)^{2},
$$

and in terms of standard generators $e_{1}, \ldots, e_{n}, f_{1}, \ldots, f_{n}$ of $(2.1)$,

$$
\left\langle e_{k}, f_{\ell}\right\rangle=e^{\delta_{k \ell} 2 \pi i / d_{k}} .
$$

The number $\nu\left(d_{1}, \ldots, d_{n}\right)$ can be computed as follows.

LEMma 2.1 (Debarre [Deb99]). We have

$$
\nu\left(d_{1}, \ldots, d_{n}\right)=\sum_{K<\mathbb{Z} / d_{1} \times \cdots \times \mathbb{Z} / d_{n}} \# \operatorname{Hom}^{\mathrm{sym}}(K, \widehat{K}),
$$

where $\widehat{K}=\operatorname{Hom}\left(K, \mathbb{C}^{*}\right)$ and Hom ${ }^{\text {sym }}$ stands for symmetric homomorphisms.

A straightforward analysis yields

$$
\nu(1, \ldots, 1, d)=\sigma(d)=\sum_{k \mid d} k
$$




\section{J. Bryan, G. Oberdieck, R. Pandharipande and Q. Yin}

A list of values of $\nu\left(d_{1}, \ldots, d_{n}\right)$ can be found in [LS02a], but some of the entries are incorrect. For example, the number $\nu(2,4)$ should be 39 and not 51 .

The counts of polarized isogenies are closely related to the counts of the lowest-genus curves on abelian surfaces and threefolds. Moreover, this lattice method is also important in counting higher-genus curves in a class of type $(1,2, d)$; see Sections 3 and 8.4.

\subsection{Reduced virtual classes}

Let $A$ be an abelian variety of dimension $n \geqslant 2$, and let $\beta \in H_{2}(A, \mathbb{Z})$ be a curve class of type

$$
(d_{1}, \ldots, d_{k}, \underbrace{0, \ldots, 0}_{m})
$$

with $d_{i}>0$ for all $i$. Here, $k>0, m \geqslant 0$, and $k+m=n$.

By the discussion in Section 2.2, there exist a subtorus $A^{\prime} \subset A$ of dimension $k$ and a curve class $\beta^{\prime} \in H_{2}\left(A^{\prime}, \mathbb{Z}\right)$ of type $\left(d_{1}, \ldots, d_{k}\right)$ such that $\beta$ is the push-forward of $\beta^{\prime}$. Write

$$
\pi: A \rightarrow A^{\prime \prime}=A / A^{\prime}
$$

for the quotient map.

Consider the moduli space of stable maps $\bar{M}_{g}(A, \beta)$. Using the cosection localization method of Kiem-Li [KL13], we define a (maximally) reduced virtual class

$$
\left[\bar{M}_{g}(A, \beta)\right]^{\mathrm{red}}
$$

on $\bar{M}_{g}(A, \beta)$. The case with marked points is done similarly. The result provides a foundation for the reduced Gromov-Witten theory of abelian varieties.

By [KL13, Section 6], every holomorphic 2-form $\theta \in H^{0}\left(A, \Omega_{A}^{2}\right)$ induces a map

$$
\sigma_{\theta}: \mathrm{Ob}_{\bar{M}_{g}(A, \beta)} \rightarrow \mathcal{O}_{\bar{M}_{g}(A, \beta)},
$$

where $\mathrm{Ob}_{\bar{M}_{g}(A, \beta)}$ is the obstruction sheaf of $\bar{M}_{g}(A, \beta)$.

Lemma 2.2. The map $\sigma_{\theta}$ is trivial if $\theta \in \pi^{*} H^{0}\left(A^{\prime \prime}, \Omega_{A^{\prime \prime}}^{2}\right)$ and surjective otherwise.

Proof. Let $[f: C \rightarrow A] \in \bar{M}_{g}(A, \beta)$ be a stable map in class $\beta$. After translation, we may assume $\operatorname{Im}(C) \subset A^{\prime}$.

By [KL13, Proposition 6.4], the map $\sigma_{\theta}$ is trivial at $[f]$ if and only if the composition

$$
\left.\left.T_{C_{\mathrm{reg}}} \stackrel{d f}{\longrightarrow} f^{*} T_{A}\right|_{C_{\mathrm{reg}}} \stackrel{f^{*} \widehat{\theta}}{\longrightarrow} f^{*} \Omega_{A}\right|_{C_{\mathrm{reg}}}
$$

is trivial. Here, $C_{\text {reg }}$ is the regular locus of $C$ and $\widehat{\theta}: T_{A} \rightarrow \Omega_{A}$ is the map induced by $\theta$. Since $\operatorname{Im}(C) \subset A^{\prime}$, it is clear that $(2.4)$ is trivial if $\theta \in \pi^{*} H^{0}\left(A^{\prime \prime}, \Omega_{A^{\prime \prime}}^{2}\right)$.

For the surjectivity statement, we identify the tangent space $T_{A, x}$ at $x \in A$ with $T_{A, 0_{A}}$ by translation. Since $\beta^{\prime}$ is of type $\left(d_{1}, \ldots, d_{k}\right)$ with $d_{i}>0$, the curve $\operatorname{Im}(C)$ generates $A^{\prime}$ as a group. By [Deb05, Lemma 8.2], there exists an open dense subset $U \subset \operatorname{Im}(C)_{\text {reg }}$ such that $T_{A^{\prime}, 0_{A^{\prime}}}$ is spanned by $T_{\operatorname{Im}(C)_{\text {reg }, x}}$ for $x \in U$. It follows that for any $\theta \in H^{0}\left(A, \Omega_{A}^{2}\right) \backslash \pi^{*} H^{0}\left(A^{\prime \prime}, \Omega_{A^{\prime \prime}}^{2}\right)$, there exists a point $x \in U$ with (2.4) non-trivial at $x$.

Hence, by taking a basis of the quotient $H^{0}\left(A, \Omega_{A}^{2}\right) / \pi^{*} H^{0}\left(A^{\prime \prime}, \Omega_{A^{\prime \prime}}^{2}\right)$, we obtain a surjective map

$$
\sigma: \mathrm{Ob}_{\bar{M}_{g}(A, \beta)} \rightarrow \mathcal{O}_{\bar{M}_{g}(A, \beta)}^{\oplus r(k, m)}
$$




\section{CURVE COUNTING ON ABELIAN SURFACES AND THREEFOLDS}

with

$$
r(k, m)=\left(\begin{array}{c}
k+m \\
2
\end{array}\right)-\left(\begin{array}{c}
m \\
2
\end{array}\right)=\frac{k(k-1)}{2}+k m .
$$

Then, by the construction of [KL13], the map $\sigma$ yields a reduced virtual class $\left[\bar{M}_{g}(A, \beta)\right]^{\text {red }}$ of dimension

$$
\operatorname{vdim} \bar{M}_{g}(A, \beta)+r(k, m)=(k+m-3)(1-g)+\frac{k(k-1)}{2}+k m .
$$

\subsection{Gromov-Witten theory in degenerate curve classes}

We now explore the possibilities of obtaining non-trivial reduced Gromov-Witten invariants for $A$ and $\beta$. By deformation invariance, the invariants depend only on the type

$$
(d_{1}, \ldots, d_{k}, \underbrace{0, \ldots, 0}_{m}) .
$$

We may then assume $A=A^{\prime} \times A^{\prime \prime}$ with $A^{\prime}$ generic among abelian varieties carrying a curve class of type $\left(d_{1}, \ldots, d_{k}\right)$ and $A^{\prime \prime}$ a product of $m$ elliptic curves, $A^{\prime \prime}=E_{1} \times \cdots \times E_{m}$. By the genericity of $A^{\prime}$, there are no stable maps of genus less than $k=\operatorname{dim} A^{\prime}$ in class $\beta$. Hence, all invariants in genus less than $k$ vanish.

We list four cases according to the number $k$ of non-zero entries in the type of $\beta$.

Case $k=1$. For $g \geqslant 1$, stable maps $[f: C \rightarrow A] \in \bar{M}_{g}(A, \beta)$ come in $m$-dimensional families via the translation action of $A^{\prime \prime}$. On the other hand, the translation by the elliptic curve $A^{\prime}$ fixes $\operatorname{Im}(f)$. The expected dimension modulo the translation by $A^{\prime \prime}$ is

$$
\operatorname{vdim} \bar{M}_{g}(A, \beta)+r(1, m)-m=(m-2)(1-g) .
$$

Integrals over the reduced class $\left[\bar{M}_{g}(A, \beta)\right]^{\text {red }}$ can be evaluated by eliminating the $E$-factors. In each step from

$$
A^{\prime} \times E_{1} \times \cdots \times E_{i+1} \text { to } A^{\prime} \times E_{1} \times \cdots \times E_{i},
$$

we find a surjective map $\mathbb{E}^{\vee} \rightarrow \mathcal{O}$, where $\mathbb{E}$ is the Hodge bundle. We then obtain a copy of the top Chern class of $\operatorname{Ker}\left(\mathbb{E}^{\vee} \rightarrow \mathcal{O}\right)$, which is $(-1)^{g-1} \lambda_{g-1}$. This follows from a close analysis of the obstruction sheaf and the definition of the reduced class. In the end, we arrive at integrals over $\left[\bar{M}_{g}\left(A^{\prime}, \beta^{\prime}\right)\right]^{\text {vir }}$ with $\left((-1)^{g-1} \lambda_{g-1}\right)^{m}$ in the integrand.

For $m=1(\operatorname{dim} A=2)$, the theory becomes the study of $\lambda_{g-1}$-integrals on the elliptic curve $A^{\prime}$. Such Hodge integrals may be expressed [FP00] in terms of the descendent theory of an elliptic curve [OP06a, OP06b].

For $m \geqslant 2(\operatorname{dim} A \geqslant 3)$, all invariants in genus $g \geqslant 2$ vanish. By Mumford's relation for $g \geqslant 2$, we have $\lambda_{g-1}^{2}=2 \lambda_{g} \lambda_{g-2}$, and $\lambda_{g}$ annihilates the virtual fundamental class of non-constant maps to the elliptic curve $A^{\prime}$. In genus 1 , all invariants are multiples of

$$
\int_{\left[\bar{M}_{1}\left(A^{\prime}, \beta^{\prime}\right)\right]^{\mathrm{vir}}} 1=\frac{\sigma\left(d_{1}\right)}{d_{1}}
$$

for $\beta^{\prime}$ of type $\left(d_{1}\right)$.

Case $k=2$. For $g \geqslant 2$, stable maps in $\bar{M}_{g}(A, \beta)$ come in $(2+m)$-dimensional families via the translation action of $A$. The expected dimension modulo translation is

$$
\operatorname{vdim} \bar{M}_{g}(A, \beta)+r(2, m)-(2+m)=(m-1)(2-g) .
$$




\section{J. Bryan, G. Oberdieck, R. Pandharipande And Q. Yin}

Similarly to the $k=1$ case, by eliminating each $E$-factor, we find a surjective map $\mathbb{E}^{\vee} \rightarrow \mathcal{O}^{\oplus 2}$ and obtain a copy of the top Chern class of $\operatorname{Ker}\left(\mathbb{E}^{\vee} \rightarrow \mathcal{O}^{\oplus 2}\right)$, which is $(-1)^{g-2} \lambda_{g-2}$.

The reduced Gromov-Witten theory of the abelian surface $A^{\prime}$ is the subject of Part I of the paper. For $m=1$ ( $\operatorname{dim} A=3)$, we find integrals of the form

$$
\int_{\left.\left[\bar{M}_{g}\left(A^{\prime}, \beta^{\prime}\right)\right]\right]^{\mathrm{red}}}(-1)^{g-2} \lambda_{g-2} \cdots,
$$

where the dots stand for further terms in the integrand. Our interest in $\lambda_{g-2}$-integrals on an abelian surface (see (1.2) and Theorem 1.1) is directly motivated by Gromov-Witten theory in degenerate curve classes on abelian threefolds.

For $m \geqslant 2(\operatorname{dim} A \geqslant 4)$, all invariants in genus $g \geqslant 3$ vanish for dimension reasons. We are then reduced to the genus 2 invariants of $A^{\prime}$ and $\beta^{\prime}$.

Case $k=3$. Similarly to the $k=2$ case, for $g \geqslant 3$, the expected dimension modulo translation is

$$
\operatorname{vdim} \bar{M}_{g}(A, \beta)+r(3, m)-(3+m)=m(3-g) .
$$

Here, by eliminating each $E$-factor, we find a surjective map $\mathbb{E}^{\vee} \rightarrow \mathcal{O}^{\oplus 3}$ and obtain a copy of the top Chern class of $\operatorname{Ker}\left(\mathbb{E}^{\vee} \rightarrow \mathcal{O}^{\oplus 3}\right)$, which is $(-1)^{g-3} \lambda_{g-3}$.

The reduced Gromov-Witten theory of the abelian threefold $A^{\prime}$ is studied in Part II of the paper. For $m \geqslant 1(\operatorname{dim} A \geqslant 4)$, all invariants in genus $g \geqslant 4$ vanish for dimension reasons. We are reduced to the genus 3 invariants of $A^{\prime}$ and $\beta^{\prime}$.

Case $k \geqslant 4$. For $g \geqslant k$, the expected dimension modulo translation is

$$
\operatorname{vdim} \bar{M}_{g}(A, \beta)+r(k, m)-(k+m)=(k-3)\left(\frac{k}{2}+1-g\right)+m(k-g) .
$$

The right-hand side of (2.6) is always negative for $g \geqslant k \geqslant 4$. Hence, all invariants vanish.

In conclusion, the (reduced) Gromov-Witten theory of abelian varieties of arbitrary dimensions is completely determined by the (reduced) Gromov-Witten theories of abelian varieties of dimension $d$ with $1 \leqslant d \leqslant 3$. The analysis here justifies our focus on these low dimensions.

Furthermore, for abelian varieties of dimension at least 4, only genus $g$ invariants with $1 \leqslant g \leqslant 3$ can possibly survive. Exact formulas are available for these invariants: for genus 1 , the formula is (2.5); for genus 2, the formula is given by Theorem 1.1; for genus 3 , the formula appears in Lemma 8.5.

\section{Part I. Abelian surfaces}

\section{The genus 2 case}

\subsection{Quotient Gromov-Witten invariants}

Let $A$ be an abelian surface, and let $\beta \in H_{2}(A, \mathbb{Z})$ be a curve class of type $\left(d_{1}, d_{2}\right)$ with $d_{1}, d_{2}>0$. In Section 1.2.1, we defined invariants

$$
\mathrm{N}_{g, \beta}^{\mathrm{FLS}}=\mathrm{N}_{g,\left(d_{1}, d_{2}\right)}^{\mathrm{FLS}}
$$




\section{CURVE COUNTING ON ABELIAN SURFACES AND THREEFOLDS}

counting genus $g$ curves in a fixed linear system. It is sometimes more natural to count curves up to translation. A reasonable path to the definition of such invariants is by integrating over the quotient stack

$$
\bar{M}_{g}(A, \beta) / A \text {. }
$$

(See Section 8.2 for such a treatment for abelian threefolds.)

Classically, people have taken a simpler course. Let

$$
p: \bar{M}_{g}(A, \beta) \rightarrow \operatorname{Pic}^{\beta}(A) \cong \widehat{A},
$$

be the morphism which sends a curve $[f: C \rightarrow A]$ to the divisor class associated with its image curve. (The construction of $p$ relies upon the Hilbert-Chow morphism). The map $p$ is equivariant with respect to the actions of $A$ on $\bar{M}_{g}(A, \beta)$ by translation and on $\widehat{A}$ by the isogeny

$$
\phi_{\beta}: A \rightarrow \widehat{A} \text {. }
$$

An element $x \in A$ fixes a linear system of type $\left(d_{1}, d_{2}\right)$ if and only if $x$ is an element of

$$
\operatorname{Ker}\left(\phi_{\beta}: A \rightarrow \widehat{A}\right) \cong\left(\mathbb{Z} / d_{1} \times \mathbb{Z} / d_{2}\right)^{2} .
$$

The quotient space (3.1) equals the quotient of $\bar{M}_{g}(A, \beta)^{\mathrm{FLS}}$ by the finite group (3.2) with $\left(d_{1} d_{2}\right)^{2}$ elements. Therefore, we define the invariants counting curves up to translation by

$$
\mathrm{N}_{g,\left(d_{1}, d_{2}\right)}^{\mathrm{Q}}=\frac{1}{\left(d_{1} d_{2}\right)^{2}} \mathrm{~N}_{g,\left(d_{1}, d_{2}\right)}^{\mathrm{FLS}}
$$

In genus 2, the invariants are related to the lattice counts considered in Section 2.3.

Lemma 3.1. For all $d_{1}, d_{2}>0$, we have $\mathrm{N}_{2,\left(d_{1}, d_{2}\right)}^{\mathrm{Q}}=\nu\left(d_{1}, d_{2}\right)$.

Proof. Let $\beta$ be of type $\left(d_{1}, d_{2}\right)$ and assume $\operatorname{End}_{\mathbb{Q}}(A)=\mathbb{Q}$. In particular, $A$ is simple (contains no elliptic curves) and $\operatorname{Aut}(A)=\{ \pm 1\}$. It follows that every genus 2 stable map $f: C \rightarrow A$ in class $\beta$ has a non-singular domain $C$. As discussed in Section 2.3, with such a map $f$, we can associate a polarized isogeny

$$
(\widehat{A}, \widehat{\beta}) \rightarrow(J, \theta)
$$

where $J$ is the Jacobian of $C$.

Conversely, every simple principally polarized abelian surface $(B, \theta)$ is the Jacobian of a unique non-singular genus 2 curve $C$. Hence, each polarized isogeny $(\widehat{A}, \widehat{\beta}) \rightarrow(B, \theta)$ induces a map

$$
f: C \stackrel{\text { aj }}{\rightarrow} B \rightarrow A .
$$

The map $f$ is unique up to translation and automorphism of $A$. Moreover, the automorphism -1 of $A$ corresponds to the hyperelliptic involution of $C$.

The abelian surface $A$ acts freely ${ }^{4}$ on $\bar{M}_{2}(A, \beta)$ by translation. To prove this, we decompose a genus 2 map $f: C \rightarrow A$ as

$$
f: C \stackrel{\text { aj }}{\rightarrow} J \stackrel{\pi}{\rightarrow} A .
$$

First, since $[\operatorname{aj}(C)]$ is a divisor class of type $(1,1)$, the only element in $J$ fixing aj $(C)$ is $0_{J}$. Second, the preimage $\pi^{-1}(f(C))$ is the union

$$
\bigcup_{x \in \operatorname{Ker}(\pi)} t_{x}(\operatorname{aj}(C))
$$

\footnotetext{
${ }^{4}$ The action is in general not free in genus greater than 2 . For example, maps from non-singular genus 3 hyperelliptic curves in a class of type $(1,2)$ have $\mathbb{Z} / 2$-stabilizers; see Sections 6.3 and 8.4 .
} 


\section{J. Bryan, G. Oberdieck, R. Pandharipande and Q. Yin}

where $t_{x}: J \rightarrow J$ is the translation by $x$. Suppose that a point $a \in A$ fixes $f(C)$, and let $b \in \pi^{-1}\{a\}$. By (3.4), we have

$$
t_{b}(\operatorname{aj}(C))=t_{x}(\operatorname{aj}(C))
$$

for some $x \in \operatorname{Ker}(\pi)$. In other words, the element $b-x \in J$ fixes aj $(C)$. Hence, $b-x=0_{J}$ and $a=\pi(b)=\pi(b-x)=0_{A}$.

It follows that $\bar{M}_{2}(A, \beta) / A$ is precisely a set of $\nu\left(d_{1}, d_{2}\right)$ isolated reduced points (or, equivalently, $\bar{M}_{2}(A, \beta)^{\mathrm{FLS}}$ is a set of $\left(d_{1} d_{2}\right)^{2} \nu\left(d_{1}, d_{2}\right)$ isolated reduced points).

\subsection{Genus 2 counts}

For genus 2, the following result determines the counts in all classes.

Theorem 3.2. For all $d_{1}, d_{2}>0$, we have

$$
\mathrm{N}_{2,\left(d_{1}, d_{2}\right)}^{\mathrm{Q}}=\sum_{k \mid \operatorname{gcd}\left(d_{1}, d_{2}\right)} \sum_{\ell \mid d_{1} d_{2} / k^{2}} k^{3} \ell
$$

The primitive case (where $\operatorname{gcd}\left(d_{1}, d_{2}\right)=1$ ) was proven in [Deb99, Göt98, LS02a] via the lattice method discussed in Section 2.3 and in [BL99, Ros14] via geometric arguments. A closer look at the lattice method actually yields a proof of Theorem 3.2 in the general case.

Proof of Theorem 3.2. To prove (3.5) for $\nu\left(d_{1}, d_{2}\right)$, we are immediately reduced to the case $\nu\left(p^{m}, p^{n}\right)$, where $p$ is a prime number and $m \leqslant n$. For $m=0$, we have, by (2.3),

$$
\nu\left(1, p^{n}\right)=\sigma\left(p^{n}\right)=\sum_{k=0}^{n} p^{k} .
$$

It then suffices to prove the following recursion:

$$
\nu\left(p^{m}, p^{n}\right)=\nu\left(1, p^{m+n}\right)+p^{3} \nu\left(p^{m-1}, p^{n-1}\right)
$$

for $1 \leqslant m \leqslant n$.

The proof uses (2.2). Consider the quotient map

$$
\pi: \mathbb{Z} / p^{m} \times \mathbb{Z} / p^{n} \rightarrow \mathbb{Z} / p^{m-1} \times \mathbb{Z} / p^{n-1} .
$$

For $1 \leqslant r \leqslant s$, the map $\pi$ induces a bijective correspondence between the following two sets:

- subgroups of $\mathbb{Z} / p^{m} \times \mathbb{Z} / p^{n}$ isomorphic to $\mathbb{Z} / p^{r} \times \mathbb{Z} / p^{s}$,

- subgroups of $\mathbb{Z} / p^{m-1} \times \mathbb{Z} / p^{n-1}$ isomorphic to $\mathbb{Z} / p^{r-1} \times \mathbb{Z} / p^{s-1}$.

We also have

$$
\begin{aligned}
& \text { \# } \operatorname{Hom}^{\operatorname{sym}}\left(\mathbb{Z} / p^{r} \times \mathbb{Z} / p^{s}, \mathbb{Z} / \widehat{p^{r} \times \mathbb{Z}} / p^{s}\right) \\
& \quad=p^{3} \# \operatorname{Hom}^{\mathrm{sym}}\left(\mathbb{Z} / p^{r-1} \times \mathbb{Z} / p^{s-1}, \mathbb{Z} / p^{r-1 \times \mathbb{Z}} / p^{s-1}\right) .
\end{aligned}
$$

The remaining subgroups of $\mathbb{Z} / p^{m} \times \mathbb{Z} / p^{n}$ are cyclic and isomorphic to $\mathbb{Z} / p^{k}$ for some $0 \leqslant k \leqslant n$. Moreover,

$$
\# \operatorname{Hom}^{\mathrm{sym}}\left(\mathbb{Z} / p^{k}, \widehat{\mathbb{Z} / p^{k}}\right)=\# \operatorname{Hom}\left(\mathbb{Z} / p^{k}, \widehat{\mathbb{Z} / p^{k}}\right)=p^{k}
$$

Applying (2.2), we find

$$
\nu\left(p^{m}, p^{n}\right)=\sum_{k=0}^{n} p^{k} \#\left\{\mathbb{Z} / p^{k}<\mathbb{Z} / p^{m} \times \mathbb{Z} / p^{n}\right\}+p^{3} \nu\left(p^{m-1}, p^{n-1}\right) .
$$




\section{CURVE COUNTING ON ABELIAN SURFACES AND THREEFOLDS}

The numbers of cyclic subgroups can be deduced from classical group theory (see [But94, Lemma 1.4.1]):

$$
\#\left\{\mathbb{Z} / p^{k}<\mathbb{Z} / p^{m} \times \mathbb{Z} / p^{n}\right\}=\sum_{\substack{0 \leqslant i \leqslant m, 0 \leqslant j \leqslant n \\ \max (i, j)=k}} \varphi\left(p^{\min (i, j)}\right),
$$

where $\varphi$ is Euler's phi function $\left(\varphi(1)=1\right.$ and $\varphi\left(p^{k}\right)=p^{k}-p^{k-1}$ for $\left.k \geqslant 1\right)$. We have

$$
\begin{aligned}
\sum_{k=0}^{n} p^{k} \#\left\{\mathbb{Z} / p^{k}<\mathbb{Z} / p^{m} \times \mathbb{Z} / p^{n}\right\} & =\sum_{k=0}^{n} p^{k} \sum_{\substack{0 \leqslant i \leqslant m, 0 \leqslant j \leqslant n \\
\max (i, j)=k}} \varphi\left(p^{\min (i, j)}\right) \\
& =\sum_{k=0}^{m} p^{k} \sum_{i=0}^{k} \varphi\left(p^{i}\right)+\sum_{k=m+1}^{n} p^{k} \sum_{i=0}^{m} \varphi\left(p^{i}\right)+\sum_{k=1}^{m} p^{k} \sum_{j=0}^{k-1} \varphi\left(p^{j}\right) \\
& =\sum_{k=0}^{m} p^{2 k}+\sum_{k=m+1}^{n} p^{m+k}+\sum_{k=1}^{m} p^{2 k-1} \\
& =\sum_{k=0}^{m+n} p^{k}=\nu\left(1, p^{m+n}\right),
\end{aligned}
$$

proving the recursion (3.6). Theorem 3.2 then follows from Lemma 3.1.

Since $g=2$ is the minimal genus for curve counting on abelian surfaces, Theorem 3.2 may be viewed as the analog of the Yau-Zaslow conjecture [YZ96] for $g=0$ counting on $K 3$ surfaces. In the $K 3$ case, primitive classes were handled first in [Bea99, BL00]. To treat the imprimitive classes, a completely new approach [KMPS10] was required (it came a decade later). For abelian surfaces, the lattice counting for the primitive case is much easier than the complete result of Theorem 3.2. The perfect matching of the lattice counts in all cases with formula of Theorem 3.2 appears miraculous.

\subsection{Multiple cover rule}

A multiple cover formula in genus $g=2$ can be extracted from Theorem 3.2. The result follows the structure of the complete multiple cover formula for $K 3$ surfaces [PT16]. We state here the multiple cover conjecture for the invariants $\mathrm{N}_{g,\left(d_{1}, d_{2}\right)}^{\mathrm{Q}}$ for all $g$.

For $d_{1}, d_{2}>0$, define the generating series of the quotient invariants:

$$
f_{\left(d_{1}, d_{2}\right)}(u)=\sum_{g \geqslant 2} \mathrm{~N}_{g,\left(d_{1}, d_{2}\right)}^{\mathrm{Q}} u^{2 g-2} .
$$

The quotient invariants are defined in terms of the FLS invariants in (3.3).

Conjecture A'. For all $d_{1}, d_{2}>0$, we have

$$
f_{\left(d_{1}, d_{2}\right)}(u)=\sum_{k \mid \operatorname{gcd}\left(d_{1}, d_{2}\right)} k f_{\left(1, d_{1} d_{2} / k^{2}\right)}(k u) .
$$

Theorem 3.2 implies the $g=2$ case of Conjecture A'. By an elementary check, Conjecture A is equivalent to Conjecture A' plus the $k=0$ case of Theorem 1.2. Since Theorem 1.2 is proven in Section 4, Conjectures A and A' are equivalent. 


\section{J. Bryan, G. Oberdieck, R. Pandharipande And Q. Yin}

\section{Primitive classes}

\subsection{Overview}

Let $A$ be an abelian surface, let $g \geqslant 2$ be the genus, and let $\beta \in H_{2}(A, \mathbb{Z})$ be a curve class of type $\left(d_{1}, d_{2}\right)$ with $d_{1}, d_{2}>0$. The class $\beta$ is primitive if $\operatorname{gcd}\left(d_{1}, d_{2}\right)=1$.

The proof of Theorem 1.2 is presented here. We proceed in two steps. First, we relate the FLS invariants to the reduced Gromov-Witten invariants of $A$ with pure point insertions and $\lambda$ classes, in Sections 4.2 and 4.3. Next, we degenerate an elliptically fibered abelian surface $A$. Using the degeneration formula in Sections 4.4 and 4.5, we reduce the calculation to an evaluation on $\mathbb{P}^{1} \times E$. The proof of Theorem 1.2 is completed in Section 4.6.

We conclude with an application in Section 4.7: a new proof is presented of a result by Göttsche and Shende [GS15] concerning the Euler characteristics of the moduli spaces of stable pairs on abelian surfaces in irreducible classes.

\subsection{Notation}

Let $\alpha \in H^{*}\left(\bar{M}_{g . n}, \mathbb{Q}\right)$ be a cohomology class on the moduli space of stable curves $\bar{M}_{g, n}$, and let $\gamma_{1}, \ldots, \gamma_{n} \in H^{*}(A, \mathbb{Q})$ be cohomology classes on $A$. The classes $\alpha$ and $\gamma_{i}$ can be pulled back to the moduli spaces

$$
\bar{M}_{g, n}(A, \beta)^{\mathrm{FLS}} \quad \text { and } \quad \bar{M}_{g, n}(A, \beta)
$$

via the forgetful map $\pi$ and the evaluation maps $\mathrm{ev}_{1}, \ldots, \mathrm{ev}_{n}$.

For each marking $i \in\{1, \ldots, n\}$, let $L_{i}$ be the associated cotangent line bundle on $\bar{M}_{g, n}(A, \beta)$. Let

$$
\psi_{i}=c_{1}\left(L_{i}\right) \in H^{2}\left(\bar{M}_{g, n}(A, \beta), \mathbb{Q}\right)
$$

be the first Chern class. Since we have the inclusion

$$
\bar{M}_{g, n}(A, \beta)^{\mathrm{FLS}} \subset \bar{M}_{g, n}(A, \beta),
$$

the classes $\psi_{i}$ restrict to $\bar{M}_{g, n}(A, \beta)^{\mathrm{FLS}}$.

The reduced Gromov-Witten invariants of $A$ are defined by

$$
\left\langle\alpha ; \tau_{a_{1}}\left(\gamma_{1}\right) \cdots \tau_{a_{n}}\left(\gamma_{n}\right)\right\rangle_{g, \beta}^{A, \mathrm{red}}=\int_{\left[\bar{M}_{g, n}(A, \beta)\right]^{\mathrm{red}}} \pi^{*}(\alpha) \prod_{i=1}^{n} \mathrm{ev}_{i}^{*}\left(\gamma_{i}\right) \psi_{i}^{a_{i}} .
$$

The FLS invariants of $A$ are defined by

$$
\left\langle\alpha ; \tau_{a_{1}}\left(\gamma_{1}\right) \cdots \tau_{a_{n}}\left(\gamma_{n}\right)\right\rangle_{g, \beta}^{A, \mathrm{FLS}}=\int_{\left[\bar{M}_{g, n}(A, \beta)^{\mathrm{FLS}}\right]_{\mathrm{red}}} \pi^{*}(\alpha) \prod_{i=1}^{n} \operatorname{ev}_{i}^{*}\left(\gamma_{i}\right) \psi_{i}^{a_{i}} .
$$

The FLS invariants can be expressed in terms of the usual invariants by a result ${ }^{5}$ of Bryan and Leung [BL99], as follows. Let

$$
\xi_{1}, \xi_{2}, \xi_{3}, \xi_{4} \in H_{1}(A, \mathbb{Z})
$$

be a basis for which the corresponding dual classes

$$
\widehat{\xi}_{1}, \widehat{\xi}_{2}, \widehat{\xi}_{3}, \widehat{\xi}_{4} \in H^{1}(\widehat{A}, \mathbb{Z}) \cong H_{1}(A, \mathbb{Z})
$$

\footnotetext{
${ }^{5}$ See [KT14, Section 4] for an algebraic proof.
} 


\section{CURVE COUNTING ON ABELIAN SURFACES AND THREEFOLDS}

satisfy the normalization

$$
\int_{\widehat{A}} \widehat{\xi}_{1} \cup \widehat{\xi}_{2} \cup \widehat{\xi}_{3} \cup \widehat{\xi}_{4}=1
$$

By first trading the higher descendents $\tau_{k}\left(\gamma_{i}\right)$ for classes pulled back from $\bar{M}_{g, n}$ up to boundary terms, we may reduce to non-gravitational insertions $\tau_{0}\left(\gamma_{i}\right)$. Then

$$
\left\langle\alpha ; \prod_{i=1}^{n} \tau_{0}\left(\gamma_{i}\right)\right\rangle_{g, \beta}^{A, \mathrm{FLS}}=\left\langle\alpha ; \prod_{i=1}^{4} \tau_{0}\left(\xi_{i}\right) \cdot \prod_{i=1}^{n} \tau_{0}\left(\gamma_{i}\right)\right\rangle_{g, \beta}^{A, \mathrm{red}},
$$

where $\alpha$ on the right side of (4.1) is viewed to be a cohomology class on $\bar{M}_{g, n+4}$ via pull-back along the map which forgets the four new points.

\subsection{Odd and even classes}

4.3.1 Trading FLS for insertions. We prove here the following simple rule which trades the FLS condition for insertions in the reduced Gromov-Witten theory of $A$.

As in Section 1.2.2, let $\mathrm{p} \in H^{4}(A, \mathbb{Z})$ be the class of a point.

Proposition 4.1. For $g \geqslant 2$ and $d_{1}, d_{2}>0$, we have

$$
\left\langle\alpha ; \tau_{0}(\mathrm{p})^{k}\right\rangle_{g,\left(d_{1}, d_{2}\right)}^{A, \mathrm{FLS}}=\frac{d_{1} d_{2}}{(k+1)(k+2)}\left\langle\alpha ; \tau_{0}(\mathrm{p})^{k+2}\right\rangle_{g,\left(d_{1}, d_{2}\right)}^{A, \text { red }}
$$

for all $\alpha \in H^{*}\left(\bar{M}_{g}, \mathbb{Q}\right)$ and $k \geqslant 0$.

The proof uses the action of $A$ on the moduli space $\bar{M}_{g, n}(A, \beta)$ to produce relations among various Gromov-Witten invariants. The argument is a modification of an elliptic vanishing argument introduced in [OP06b].

4.3.2 Abelian vanishing. Let $\beta \in H_{2}(A, \mathbb{Z})$ be any curve class. For $n \geqslant 1$, let

$$
\mathrm{ev}_{1}: \bar{M}_{g, n}(A, \beta) \rightarrow A
$$

be the first evaluation map. Denote the fiber of the identity element $0_{A} \in A$ by

$$
\bar{M}_{g, n}^{0}(A, \beta)=\mathrm{ev}_{1}^{-1}\left(0_{A}\right) .
$$

We have the product decomposition

$$
\bar{M}_{g, n}(A, \beta)=\bar{M}_{g, n}^{0}(A, \beta) \times A .
$$

The reduced virtual class on $\bar{M}_{g, n}(A, \beta)$ is pulled back from a class on $\bar{M}_{g, n}^{0}(A, \beta)$. Consider the commutative diagram

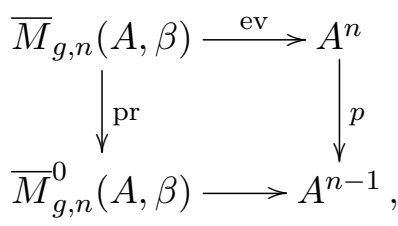

where pr is projection onto the first factor of (4.2) and

$$
p\left(x_{1}, \ldots, x_{n}\right)=\left(x_{2}-x_{1}, \ldots, x_{n}-x_{1}\right) .
$$




\section{J. Bryan, G. Oberdieck, R. Pandharipande And Q. Yin}

Lemma 4.2. Let $\alpha \in H^{*}\left(\bar{M}_{g, n}, \mathbb{Q}\right)$ and $\gamma \in H^{*}\left(A^{n-1}, \mathbb{Q}\right)$ be arbitrary classes. For any $\gamma_{1} \in$ $H^{*}(A, \mathbb{Q})$ of degree $\operatorname{deg}\left(\gamma_{1}\right) \leqslant 3$, we have

$$
\int_{\left[\bar{M}_{g, n}(A, \beta)\right]^{\operatorname{red}}} \pi^{*}(\alpha) \cup \operatorname{ev}_{1}^{*}\left(\gamma_{1}\right) \cup \mathrm{ev}^{*} p^{*}(\gamma)=0
$$

Proof. The class

$$
\left(\pi^{*}(\alpha) \cup \mathrm{ev}^{*} p^{*}(\gamma)\right) \cap\left[\bar{M}_{g, n}(A, \beta)\right]^{\mathrm{red}}
$$

is the pull-back via pr of a class $\theta$ on $\bar{M}_{g, n}^{0}(A, \beta)$. Using the push-pull formula, we have

$$
\operatorname{pr}_{*}\left(\operatorname{ev}_{1}^{*}\left(\gamma_{1}\right) \cap \operatorname{pr}^{*}(\theta)\right)=\operatorname{pr}_{*} \operatorname{ev}_{1}^{*}\left(\gamma_{1}\right) \cap \theta=0 .
$$

The last equality holds for dimension reasons since $\gamma_{1} \in H^{\leqslant 3}(A, \mathbb{Q})$ and the fibers of pr are $A$.

4.3.3 Proof of Proposition 4.1. We study the split abelian surface $A=E_{1} \times E_{2}$, where $E_{1}$ and $E_{2}$ are two generic elliptic curves. Consider the curve class

$$
\left(d_{1}, d_{2}\right)=d_{1}\left[E_{1}\right]+d_{2}\left[E_{2}\right] \in H_{2}(A, \mathbb{Z}) .
$$

For $i \in\{1,2\}$, let $\omega_{i} \in H^{2}\left(E_{i}, \mathbb{Z}\right)$ be the class of a point on $E_{i}$, and let $\mathrm{a}_{i}, \mathrm{~b}_{i} \in H^{1}\left(E_{i}, \mathbb{Z}\right)$ be a symplectic basis. We use freely the identification induced by the Künneth decomposition

$$
H^{*}\left(E_{1} \times E_{2}, \mathbb{Z}\right)=H^{*}\left(E_{1}, \mathbb{Z}\right) \otimes H^{*}\left(E_{2}, \mathbb{Z}\right) .
$$

The proof of Proposition 4.1 follows directly from (4.1) and the following two lemmas.

Lemma 4.3. For $\alpha \in H^{*}\left(\bar{M}_{g}, \mathbb{Q}\right)$, we have

$$
d_{2}\left\langle\alpha ; \tau_{0}(\mathrm{p})^{k+2}\right\rangle_{g,\left(d_{1}, d_{2}\right)}^{A, \text { red }}=(k+2) \cdot\left\langle\alpha ; \tau_{0}\left(\mathrm{a}_{1} \omega_{2}\right) \tau_{0}\left(\mathrm{~b}_{1} \omega_{2}\right) \tau_{0}(\mathrm{p})^{k+1}\right\rangle_{g,\left(d_{1}, d_{2}\right)}^{A, \text { red }} .
$$

Proof. Consider the class $\gamma=\mathrm{b}_{1} \omega_{2} \otimes \mathrm{p}^{\otimes k+1} \in H^{*}\left(A^{k+2}, \mathbb{Q}\right)$. In the notation of Section 4.3.2 for $n=k+3$, we have

$$
p^{*}\left(1^{\otimes i-1} \otimes v \otimes 1^{\otimes k+2-i}\right)=-v \otimes 1^{\otimes k+2}+1^{\otimes i} \otimes v \otimes 1^{\otimes k+2-i}
$$

for all $i=1, \ldots, k+2$ and all $v \in\left\{\mathrm{a}_{1}, \mathrm{~b}_{1}, \mathrm{a}_{2}, \mathrm{~b}_{2}\right\}$.

Denote the projection onto the first factor of $A^{k+3}$ by $\pi_{1}: A^{k+3} \rightarrow A$. Let $u=\mathrm{a}_{1} \omega_{2}$. Via several applications of (4.3), we find

$$
\begin{aligned}
\pi_{1}^{*}(u) \cup p^{*}(\gamma)= & \mathrm{a}_{1} \omega_{2} \otimes \mathrm{b}_{1} \omega_{2} \otimes \mathrm{p}^{\otimes k+1}-\mathrm{p} \otimes \omega_{2} \otimes \mathrm{p}^{\otimes k+1} \\
& -\sum_{i=1}^{k+1} \mathrm{p} \otimes \mathrm{b}_{1} \omega_{2} \otimes \mathrm{p}^{\otimes i-1} \otimes \mathrm{a}_{1} \omega_{2} \otimes \mathrm{p}^{\otimes k+1-i} .
\end{aligned}
$$

After applying the abelian vanishing of Lemma 4.2, we obtain

$$
\left\langle\alpha ; \tau_{0}\left(\omega_{2}\right) \tau_{0}(\mathrm{p})^{k+2}\right\rangle_{g,\left(d_{1}, d_{2}\right)}^{A, \text {,ed }}=(k+2)\left\langle\alpha ; \tau_{0}\left(\mathrm{a}_{1} \omega_{2}\right) \tau_{0}\left(\mathrm{~b}_{1} \omega_{2}\right) \tau_{0}(\mathrm{p})^{k+1}\right\rangle_{g,\left(d_{1}, d_{2}\right)}^{A, \text { red }} .
$$

The lemma then follows from the divisor equation.

Lemma 4.4. For $\alpha \in H^{*}\left(\bar{M}_{g}, \mathbb{Q}\right)$, we have

$$
\begin{aligned}
& d_{1}\left\langle\alpha ; \tau_{0}\left(\mathrm{a}_{1} \omega_{2}\right) \tau_{0}\left(\mathrm{~b}_{1} \omega_{2}\right) \tau_{0}(\mathrm{p})^{k+1}\right\rangle_{g,\left(d_{1}, d_{2}\right)}^{A, \text { red }} \\
& \quad=(k+1)\left\langle\alpha ; \tau_{0}\left(\mathrm{a}_{1} \omega_{2}\right) \tau_{0}\left(\mathrm{~b}_{1} \omega_{2}\right) \tau_{0}\left(\omega_{1} \mathrm{a}_{2}\right) \tau_{0}\left(\omega_{1} \mathrm{~b}_{2}\right) \tau_{0}(\mathrm{p})^{k}\right\rangle_{g,\left(d_{1}, d_{2}\right)}^{A, \text { red }} .
\end{aligned}
$$




\section{CURVE COUNTING ON ABELIAN SURFACES AND THREEFOLDS}

Proof. Consider the class $\gamma=\omega_{1} \mathrm{~b}_{2} \otimes \mathrm{a}_{1} \omega_{2} \otimes \mathrm{b}_{1} \omega_{2} \otimes \mathrm{p}^{\otimes k}$, and let $u=\omega_{1} \mathrm{a}_{2}$. We apply the abelian vanishing just as in the proof of Lemma 4.3. Every term with an insertion of the form $\tau_{0}(v w)$ for $v \in\left\{\mathrm{a}_{1}, \mathrm{~b}_{1}\right\}$ and $w \in\left\{\mathrm{a}_{2}, \mathrm{~b}_{2}\right\}$ contributes 0 by the divisor equation. We obtain

$$
\begin{aligned}
& \left\langle\alpha ; \tau_{0}\left(\omega_{1}\right) \tau_{0}\left(\mathrm{a}_{1} \omega_{2}\right) \tau_{0}\left(\mathrm{~b}_{1} \omega_{2}\right) \tau_{0}(\mathrm{p})^{k+1}\right\rangle_{g,\left(d_{1}, d_{2}\right)}^{A, \text { red }} \\
& \quad=(k+1)\left\langle\alpha ; \tau_{0}\left(\mathrm{a}_{1} \omega_{2}\right) \tau_{0}\left(\mathrm{~b}_{1} \omega_{2}\right) \tau_{0}\left(\omega_{1} \mathrm{a}_{2}\right) \tau_{0}\left(\omega_{1} \mathrm{~b}_{2}\right) \tau_{0}(\mathrm{p})^{k}\right\rangle_{g,\left(d_{1}, d_{2}\right)}^{A, \text { red }},
\end{aligned}
$$

which yields the result by an application of the divisor equation.

\subsection{Degeneration formula}

Let the abelian surface $A=E_{1} \times E_{2}$ be the product of two generic elliptic curves $E_{1}$ and $E_{2}$, and let $E=0_{E_{1}} \times E_{2}$ be a fixed fiber of the projection to $E_{1}$. The degeneration of $A$ to the normal cone of $E \subset A$ is the family

$$
\epsilon: X=\mathrm{Bl}_{E \times 0}\left(A \times \mathbb{P}^{1}\right) \rightarrow \mathbb{P}^{1} .
$$

For $\xi \in \mathbb{P}^{1} \backslash\{0\}$, the fiber $X_{\xi}=\epsilon^{-1}(\xi)$ is isomorphic to $A$. For $\xi=0$, we have

$$
X_{0}=A \cup_{E}\left(\mathbb{P}^{1} \times E\right) .
$$

We will apply the degeneration formula of Gromov-Witten theory [Li01, Li02] to the family (4.4).

For our use, the degeneration formula must be modified for the reduced virtual class. More precisely, the degeneration formula expresses the reduced Gromov-Witten theory of $A$ in terms of the reduced relative Gromov-Witten theory of $A / E$ and standard relative Gromov-Witten theory of $\left(\mathbb{P}^{1} \times E\right) / E$. The technical point in the modification of the degeneration formula is to define a reduced virtual class on the moduli space

$$
\bar{M}_{g, n}(\epsilon, \beta)
$$

of stable maps to the fibers of $\epsilon$. Note that every fiber $X_{\xi}$ and every expanded degeneration $\widehat{X}_{\xi}$ maps to the abelian surface $A$. The pull-back of the symplectic form of $A$ to $\widehat{X}_{\xi}$ then yields a 2-form on $\widehat{X}_{\xi}$ which vanishes on all components except $A$. With the usual arguments [MP13, MPT10], we obtain a quotient of the obstruction sheaf which only changes the obstruction sheaf on the $A$ side. The outcome is the desired degeneration formula. A parallel argument can be found in [MPT10, Section 6].

\subsection{The surface $\mathbb{P}^{1} \times E$}

For the trivial elliptic fibrations

$$
p: A \rightarrow E_{1} \text { and } \widehat{p}: \mathbb{P}^{1} \times E \rightarrow \mathbb{P}^{1},
$$

we denote the section class by $B$ and the fiber class by $E$. We also write

$$
\left(d_{1}, d_{2}\right)=d_{1} B+d_{2} E
$$

for the corresponding classes in $H_{2}(A, \mathbb{Z})$ and $H_{2}\left(\mathbb{P}^{1} \times E, \mathbb{Z}\right)$.

We will use the standard bracket notation

$$
\left\langle\mu\left|\alpha \prod_{i} \tau_{a_{i}}\left(\gamma_{i}\right)\right| \nu\right\rangle_{g,(1, d)}^{\mathbb{P}^{1} \times E}=\int_{\left[\bar{M}_{g, n}\left(\left(\mathbb{P}^{1} \times E\right) /\{0, \infty\},(1, d)\right)_{\mu, \nu}\right] \mathrm{vir}} \alpha \cup \prod_{i} \psi_{i}^{a_{i}} \mathrm{ev}_{i}^{*}\left(\gamma_{i}\right)
$$

for the Gromov-Witten invariants of $\mathbb{P}^{1} \times E$ relative to the fibers over $0, \infty \in \mathbb{P}^{1}$. The integral 


\section{J. Bryan, G. Oberdieck, R. Pandharipande and Q. Yin}

is over the moduli space of stable maps

$$
\bar{M}_{g, n}\left(\left(\mathbb{P}^{1} \times E\right) /\{0, \infty\},(1, d)\right)
$$

relative to the fibers over $0, \infty \in \mathbb{P}^{1}$ in class $(1, d)$. Here,

$$
\mu \in H^{*}(0 \times E) \text { and } \quad \nu \in H^{*}(\infty \times E)
$$

are cohomology classes on the relative divisors. The integrand contains $\alpha \in H^{*}\left(\bar{M}_{g, n}, \mathbb{Q}\right)$ and the descendents.

We form the generating series of relative invariants

$$
\left\langle\mu\left|\alpha \prod_{i} \tau_{a_{i}}\left(\gamma_{i}\right)\right| \nu\right\rangle^{\mathbb{P}^{1} \times E}=\sum_{g \geqslant 0} \sum_{d \geqslant 0} u^{2 g-2} q^{d}\left\langle\mu\left|\alpha \prod_{i} \tau_{a_{i}}\left(\gamma_{i}\right)\right| \nu\right\rangle_{g,(1, d)}^{\mathbb{P}^{1} \times E} .
$$

Similar definitions apply also to the case of a single relative divisor and to the case of the abelian surface $A$ (with respect to the reduced virtual class).

We will require several exact evaluations. Let

$$
\mathbb{E}^{\vee}(1)=c\left(\mathbb{E}^{\vee}\right)
$$

denote the total Chern class of the dual of the Hodge bundle, and let $\omega$ be the class of a point on the relative divisors of $A$ and $E \times \mathbb{P}^{1}$.

LEMMA 4.5. We have

$$
\begin{aligned}
\left\langle 1\left|\mathbb{E}^{\vee}(1) \tau_{0}(\mathrm{p})\right| 1\right\rangle^{\mathbb{P}^{1} \times E} & =\frac{1}{u^{2}}, \\
\left\langle 1\left|\mathbb{E}^{\vee}(1) \tau_{0}(\mathrm{p})\right| \omega\right\rangle^{\mathbb{P}^{1} \times E} & =\frac{1}{u^{2}} \sum_{d \geqslant 1} \sum_{m \mid d} \frac{d}{m}(2 \sin (m u / 2))^{2} q^{d}, \\
\left\langle\omega\left|\mathbb{E}^{\vee}(1) \tau_{0}(\mathrm{p})\right| \omega\right\rangle^{\mathbb{P}^{1} \times E} & =0 .
\end{aligned}
$$

Proof. The first equation is obtained by exactly following the proof of [MPT10, Lemma 24]. The second equation follows from [MPT10, Lemmas 25 and 26]. For the third equality, the point conditions on the relative divisors can be chosen to be different. Then, since the degree over $\mathbb{P}^{1}$ is 1 (and there are no non-constant maps from $\mathbb{P}^{1}$ to $E$ ), the moduli space with the relative conditions imposed is empty.

Lemma 4.6. For $g \geqslant 0$ and $d \geqslant 0$, we have $\left\langle\mathbb{E}^{\vee}(1) \mid \omega\right\rangle_{g,(1, d)}^{A, \text { red }}=\delta_{g, 1} \delta_{d, 0}$.

Proof. By dimension reasons, only the term $(-1)^{g-1} \lambda_{g-1}=c_{g-1}\left(\mathbb{E}^{\vee}\right)$ contributes in the evaluation of Lemma 4.6.

Case $d>0$. We will prove the vanishing of $\lambda_{g-1}$ on $\bar{M}_{g}(A / E, \beta)$ by giving two linearly independent sections of $\mathbb{E}$.

Let $\gamma, \gamma^{\prime} \in H^{0}\left(A, \Omega_{A}\right)$ be the pull-backs to $A$ of non-zero global differential forms on $E_{1}$ and $E_{2}$, respectively. Let $\pi: \mathcal{C} \rightarrow \bar{M}_{g}(A / E, \beta)$ be the universal curve, and let $f: \mathcal{C} \rightarrow A$ be the universal map. We have the induced sequence

$$
\mathcal{O}_{\mathcal{C}}^{2} \stackrel{\left(\gamma, \gamma^{\prime}\right)}{\longrightarrow} f^{*} \Omega_{A} \rightarrow \Omega_{\pi} \rightarrow \omega_{\pi}
$$

By push-forward via $\pi$, we obtain the sequence

$$
s: \mathcal{O}_{M_{g}(A / E, \beta)}^{2} \rightarrow \pi_{*} \omega_{\pi}=\mathbb{E} .
$$




\section{Curve Counting on ABElian SURFACES AND ThreEFoldS}

If $s$ does not define a 2-dimensional subbundle of $\mathbb{E}$, there exist a map $f_{0}: C \rightarrow A$ in class $(1, d)$ and non-zero elements $c, c^{\prime} \in \mathbb{C}$ such that

$$
f_{0}^{*}\left(c \gamma+c^{\prime} \gamma^{\prime}\right)=0 \text {. }
$$

Thus $f_{0}$ must map to a (translate of a) 1-dimensional abelian subvariety $V$ inside $E_{1} \times E_{2}$. Because $f$ has degree $(1, d)$, the subvariety $V$ induces an isogeny between $E_{1}$ and $E_{2}$. But $E_{1}$ and $E_{2}$ were chosen generic, which gives a contradiction. Hence $s$ is injective and $\lambda_{g-1}=0$.

Case $d=0$. We have the factor $R^{1} \pi_{*}\left(f^{*} T_{E_{2}}\right)$ inside the obstruction sheaf, which yields an additional class $(-1)^{g-1} \lambda_{g-1}$ after reduction. Therefore, we have

$$
\left\langle\mathbb{E}^{\vee}(1) \mid \omega\right\rangle_{g,(1,0)}^{A, \mathrm{red}}=\int_{\left[\bar{M}_{g}\left(E_{1} / 0,1\right)\right]^{\mathrm{vir}}} \lambda_{g-1}^{2} .
$$

For $g \geqslant 2$, we have $\lambda_{g-1}^{2}=2 \lambda_{g} \lambda_{g-2}$ by Mumford's relation. By pulling back the global non-zero 1-form from $E_{1}$, we obtain a 1-dimensional subbundle of $\mathbb{E}$. Therefore, we have $\lambda_{g}=0$ and the integral vanishes.

Finally, for $g=1$ and $d=0$, the moduli space $\bar{M}_{1}\left(E_{1} / 0,1\right)$ is a single point and the invariant is 1 .

\subsection{Proof of Theorem $\mathbf{1 . 2}$}

We are now able to evaluate the invariants $\mathrm{N}_{g, k,(1, d)}^{\mathrm{FLS}}$ of Section 1.2.2 and prove Theorem 1.2. By definition,

$$
\sum_{g \geqslant 2} \sum_{d \geqslant 1} \mathrm{~N}_{g, k,(1, d)}^{\mathrm{FLS}} u^{2 g-2} q^{d}=\sum_{g \geqslant 2} \sum_{d \geqslant 1} u^{2 g-2} q^{d}\left\langle\mathbb{E}^{\vee}(1) \tau_{0}(\mathrm{p})^{k}\right\rangle_{g,(1, d)}^{A, \mathrm{FLS}} .
$$

By Proposition 4.1, the right side of (4.5) equals

$$
q \frac{d}{d q} \frac{1}{(k+1)(k+2)} \sum_{g \geqslant 0} \sum_{d \geqslant 0} u^{2 g-2} q^{d}\left\langle\mathbb{E}^{\vee}(1) \tau_{0}(\mathrm{p})^{k+2}\right\rangle_{g,(1, d)}^{A, \text { red }} .
$$

Next, we apply the degeneration formula. Only one term satisfies the dimension constraints:

$$
q \frac{d}{d q} \frac{u^{2}}{(k+1)(k+2)} \cdot\left\langle\mathbb{E}^{\vee}(1) \mid \omega\right\rangle^{A, \text { red }} \cdot\left\langle 1 \mid \mathbb{E}^{\vee}(1) \tau_{0}(\mathrm{p})^{k+2}\right\rangle^{\mathbb{P}^{1} \times E}
$$

An application of Lemma 4.6 then yields

$$
q \frac{d}{d q} \frac{u^{2}}{(k+1)(k+2)} \cdot\left\langle 1 \mid \mathbb{E}^{\vee}(1) \tau_{0}(\mathrm{p})^{k+2}\right\rangle^{\mathbb{P}^{1} \times E} .
$$

We degenerate the base $\mathbb{P}^{1}$ to obtain a chain of $k+3$ surfaces isomorphic to $\mathbb{P}^{1} \times E$. The first $k+2$ of these each receive a single insertion $\tau_{0}(\mathrm{p})$. Using the vanishing of Lemma 4.5 and the evaluation of the last $\mathbb{P}^{1} \times E$ by [MPT10, Lemma 24], we obtain

$$
q \frac{d}{d q} \frac{u^{2}(k+2)}{(k+1)(k+2)} \cdot\left\langle 1\left|\mathbb{E}^{\vee}(1) \tau_{0}(\mathrm{p})\right| 1\right\rangle^{\mathbb{P}^{1} \times E} \cdot\left(u^{2}\left\langle 1\left|\mathbb{E}^{\vee}(1) \tau_{0}(\mathrm{p})\right| \omega\right\rangle^{\mathbb{P}^{1} \times E}\right)^{k+1}
$$

A further application of Lemma 4.5 yields

$$
q \frac{d}{d q} \frac{1}{k+1}\left(\sum_{d \geqslant 1} \sum_{m \mid d} \frac{d}{m}(2 \sin (m u / 2))^{2} q^{d}\right)^{k+1} .
$$




\section{J. Bryan, G. Oberdieck, R. Pandharipande And Q. Yin}

Rewriting the result in the variables $p=e^{2 \pi i z}, q=e^{2 \pi i \tau}$, and $u=2 \pi z$, we obtain

$$
q \frac{d}{d q} \frac{1}{k+1}\left(-\sum_{d \geqslant 1} \sum_{m \mid d} \frac{d}{m}\left(p^{m}-2+p^{-m}\right) q^{d}\right)^{k+1}=q \frac{d}{d q} \frac{1}{k+1} \mathrm{~S}(z, \tau)^{k+1} .
$$

The proof of Theorem 1.2 is thus complete.

\subsection{Relation to stable-pairs invariants}

Let $A$ be an abelian surface, and let $\beta$ be an irreducible curve class of type $(1, d)$. Let $P_{n}(A, \beta)$ be the moduli space of stable pairs $(F, s)$ on $A$ in class $\beta$ and with Euler characteristic $\chi(F)=n$; see [PT09]. The moduli spaces $P_{n}(A, \beta)$ are isomorphic to the relative Hilbert scheme over the universal family of curves in class $\beta$. It is non-singular of dimension $2 d+n+1$.

Consider the Hilbert-Chow map

$$
p: P_{n}(A, \beta) \rightarrow \operatorname{Pic}^{\beta}(A) \cong \widehat{A},
$$

which sends a stable pair $(F, s)$ to the divisor class associated with the support of $F$. The map is equivariant with respect to the action of $A$ and is an isotrivial étale fibration. The fiber of $p$ over $0_{\widehat{A}}$ is denoted by

$$
P_{n}(A, \beta)^{\mathrm{FLS}}=p^{-1}\left(0_{\widehat{A}}\right) .
$$

We define the FLS stable-pairs invariants in class $\beta$ to be the signed Euler characteristic

$$
\mathrm{P}_{n, \beta}^{\mathrm{FLS}}=(-1)^{2 d+n-1} e\left(P_{n}(A, \beta)^{\mathrm{FLS}}\right) .
$$

The definition agrees with the definition of residue stable-pairs invariants of the threefold $A \times \mathbb{C}$ using torus localization; see [MPT10].

By the invariance of the Euler characteristic under deformations with smooth fibers, $\mathrm{P}_{n, \beta}^{\mathrm{FLS}}$ only depends on the type $(1, d)$ of the irreducible class $\beta$. We write

$$
\mathrm{P}_{n, \beta}^{\mathrm{FLS}}=\mathrm{P}_{n,(1, d)}^{\mathrm{FLS}} .
$$

The Euler characteristics (4.6) (in fact the $\chi_{y}$-genus) have been computed by Göttsche and Shende.

TheOREM 4.7 (Göttsche-Shende [GS15]). We have

$$
\sum_{d \geqslant 1} \sum_{n \in \mathbb{Z}} \mathrm{P}_{n,(1, d)}^{\mathrm{FLS}}(-p)^{n} q^{d}=-\sum_{d \geqslant 1} \sum_{m \mid d} \frac{d^{2}}{m}\left(p^{m}-2+p^{-m}\right) q^{d} .
$$

The $k=0$ case of Theorem 1.2 yields a second proof of this result: using an analog of the abelian vanishing relation (Lemma 4.2) for stable pairs, we may express the FLS condition by point insertions on the full moduli space $P_{n}(A, \beta)$, as in Proposition 4.1. After degenerating the abelian surface $A$ to $\mathbb{P}^{1} \times E$, we can apply the Gromov-Witten/Pairs correspondence [PP17], which yields the result.

In [MPT10], a parallel study of the reduced invariants of $K 3$ surfaces was undertaken. The Gromov-Witten/Pairs correspondence and the Euler characteristic calculations of KawaiYoshioka [KY00] were used together to evaluate the Gromov-Witten side to prove the primitive Katz-Klemm-Vafa conjecture. The analog of the Kawai-Yoshioka calculation for abelian surfaces is Theorem 4.7. However, for abelian surfaces, we are able to evaluate the Gromov-Witten side directly without using input from the stable-pairs side. 


\section{CURVE COUNTING ON ABELIAN SURFACES AND THREEFOLDS}

\section{Quasi-modular forms}

\subsection{Descendent series}

Let $A=E_{1} \times E_{2}$ be the product of two generic elliptic curves $E_{1}$ and $E_{2}$, and let

$$
\left(d_{1}, d_{2}\right)=d_{1}\left[E_{1}\right]+d_{2}\left[E_{2}\right] \in H_{2}(A, \mathbb{Z}) .
$$

For $i \in\{1,2\}$, let $\omega_{i} \in H^{2}\left(E_{i}, \mathbb{Z}\right)$ be the class of a point on $E_{i}$ and let $\mathrm{a}_{i}, \mathrm{~b}_{i} \in H^{1}\left(E_{i}, \mathbb{Z}\right)$ be a symplectic basis. As before, we use freely the identification induced by the Künneth decomposition

$$
H^{*}\left(E_{1} \times E_{2}, \mathbb{Z}\right)=H^{*}\left(E_{1}, \mathbb{Z}\right) \otimes H^{*}\left(E_{2}, \mathbb{Z}\right) .
$$

A class $\gamma \in H^{*}(A, \mathbb{Q})$ is monomial if $\gamma$ is a product

$$
\gamma=\mathrm{a}_{1}^{i} \mathrm{~b}_{1}^{j} \mathrm{a}_{2}^{k} \mathrm{~b}_{2}^{l}, \quad i, j, k, l \in\{0,1\} .
$$

A basis of the cohomology of $A$ is given by monomial classes. For a monomial class $\gamma$, we denote by $v_{s}(\gamma)$ the exponent of $s \in\left\{\mathrm{a}_{1}, \mathrm{~b}_{1}, \mathrm{a}_{2}, \mathrm{~b}_{2}\right\}$ in $\gamma$. Hence,

$$
\gamma=a_{1}^{v_{a_{1}}(\gamma)} b_{1}^{v_{b_{1}}(\gamma)} a_{2}^{v_{\mathrm{a}_{2}}(\gamma)} b_{2}^{v_{\mathrm{b}_{2}}(\gamma)} .
$$

Let $\gamma_{1}, \ldots, \gamma_{n} \in H^{*}(A, \mathbb{Q})$ be monomial classes, and let $a_{1}, \ldots, a_{n} \in \mathbb{Z}^{\geqslant 0}$ be non-negative integers. The primitive descendent potential of $A$ with insertions $\tau_{a_{1}}\left(\gamma_{1}\right), \ldots, \tau_{a_{n}}\left(\gamma_{n}\right)$ is defined by

$$
\mathrm{F}_{g}^{A}\left(\tau_{a_{1}}\left(\gamma_{1}\right) \cdots \tau_{a_{n}}\left(\gamma_{n}\right)\right)=\sum_{d \geqslant 0}\left\langle\tau_{a_{1}}\left(\gamma_{1}\right) \cdots \tau_{a_{n}}\left(\gamma_{n}\right)\right\rangle_{g,(1, d)}^{A, \text { red }} q^{d}
$$

Theorem 1.3 states a modularityproperty for $\mathrm{F}_{g}^{A}\left(\tau_{a_{1}}\left(\gamma_{1}\right) \cdots \tau_{a_{n}}\left(\gamma_{n}\right)\right)$. (The vector space of quasimodular forms was defined in Section 1.2.3.) The following refined result will be proven here.

THEOREM 5.1. The primitive descendent potential satisfies the following properties for all $g \geqslant 1$ :

(i) The series $\mathrm{F}_{g}^{A}\left(\tau_{a_{1}}\left(\gamma_{1}\right) \cdots \tau_{a_{n}}\left(\gamma_{n}\right)\right)$ vanishes unless

$$
\sum_{k=1}^{n} v_{\mathrm{a}_{i}}\left(\gamma_{k}\right)=\sum_{k=1}^{n} v_{\mathrm{b}_{i}}\left(\gamma_{k}\right), \quad i \in\{1,2\} .
$$

(ii) We have $\mathrm{F}_{g}^{A}\left(\tau_{a_{1}}\left(\gamma_{1}\right) \cdots \tau_{a_{n}}\left(\gamma_{n}\right)\right) \in \operatorname{QMod}_{2(g-2)+2 \ell}$ for $\ell=\sum_{k=1}^{n} v_{\mathrm{a}_{2}}\left(\gamma_{k}\right)$.

Part (i) of Theorem 5.1 is a basic balancing condition. Part (ii) is a homogeneity property which refines the statement of Theorem 1.3.

Having the precise weight is useful in applications. For example, by part (ii) of Theorem 5.1, the series $\mathrm{F}_{2}^{A}\left(\tau_{1}(\mathrm{p})\right)$ is a quasi-modular form of weight 2 , and hence a multiple of $E_{2}(\tau)$. The constant coefficient is given by

$$
\int_{\left[\bar{M}_{2,1}(A,(1,0))\right]^{\mathrm{red}}} \mathrm{ev}_{1}^{*}(\mathrm{p}) \psi_{1}=\int_{\left[\bar{M}_{2,1}\left(E_{1}, 1\right)\right]^{\mathrm{vir}}}\left(-\lambda_{1}\right) \operatorname{ev}_{1}^{*}\left(\omega_{1}\right) \psi_{1}=-\frac{1}{12} .
$$

We conclude

$$
\mathrm{F}_{2}^{A}\left(\tau_{1}(\mathrm{p})\right)=-\frac{1}{12} E_{2}(\tau)=-\frac{1}{12}+2 \sum_{d \geqslant 0} \sigma(d) q^{d} .
$$

For genus $g=1$, both parts of Theorem 5.1 are easy to see. The contributions of curve classes of type $(1, d)$ with $d>0$ vanish for $g=1$ since the moduli space of maps is empty: an element of

$$
\bar{M}_{1, n}\left(E_{1} \times E_{2},(1, d)\right), \quad d>0,
$$




\section{J. Bryan, G. Oberdieck, R. Pandharipande And Q. Yin}

would yield an isogeny between $E_{1}$ and $E_{2}$, contradicting the genericity of $E_{1}$ and $E_{2}$. The series $\mathrm{F}_{1}^{A}\left(\tau_{a_{1}}\left(\gamma_{1}\right) \cdots \tau_{a_{n}}\left(\gamma_{n}\right)\right)$ therefore has only a constant term in $q$. For curve classes of degree $(1,0)$, the moduli space factors as

$$
\bar{M}_{1, n}\left(E_{1} \times E_{2},(1,0)\right) \cong \bar{M}_{1, n}\left(E_{1}, 1\right) \times E_{2} .
$$

The balancing condition of part (i) then follows by the separate balancing on the two factors. For non-vanishing invariants $\left\langle\tau_{a_{1}}\left(\gamma_{1}\right) \cdots \tau_{a_{n}}\left(\gamma_{n}\right)\right\rangle_{1,(1,0)}^{A \text {,red }}$, we must have $\ell=\sum_{k=1}^{n} v_{\mathrm{a}_{2}}\left(\gamma_{k}\right)=1$. Hence, part (ii) correctly predicts a quasi-modular form of weight

$$
2(g-2)+2 \ell=2(1-2)+2 \cdot 1=0 .
$$

\subsection{Tautological classes}

The first step in the proof of Theorem 5.1 is to recast the result in terms of tautological classes on the moduli space of curves. For $2 g-2+n>0$, let $R^{*}\left(\bar{M}_{g, n}\right) \subset H^{*}\left(\bar{M}_{g, n}, \mathbb{Q}\right)$ be the tautological ring. Let $\pi: \bar{M}_{g, n}(A, \beta) \rightarrow \bar{M}_{g, n}$ be the forgetful map. For $\alpha \in R^{*}\left(\bar{M}_{g, n}\right)$, we define $\alpha$-twisted reduced invariants of $A$ by

$$
\left\langle\alpha ; \gamma_{1}, \ldots, \gamma_{n}\right\rangle_{g, \beta}^{A, \text { red }}=\int_{\left[\bar{M}_{g, n}(A, \beta)\right]^{\mathrm{red}}} \pi^{*}(\alpha) \cup \prod_{i} \operatorname{ev}_{i}^{*}\left(\gamma_{i}\right) .
$$

Here, $\beta$ is a curve class on $A$ and $\gamma_{1}, \ldots, \gamma_{n} \in H^{*}(A, \mathbb{Q})$ are monomial classes. The associated primitive potential is defined by

$$
\mathrm{F}_{g}^{A}\left(\alpha ; \gamma_{1}, \ldots, \gamma_{n}\right)=\sum_{d \geqslant 0}\left\langle\alpha ; \gamma_{1}, \ldots, \gamma_{n}\right\rangle_{g,(1, d)}^{A, \text { red }} q^{d}
$$

Proposition 5.2. The primitive $\alpha$-twisted potential satisfies the following properties for all $g \geqslant 1$ :

(i) The series $\mathrm{F}_{g}^{A}\left(\alpha ; \gamma_{1}, \ldots, \gamma_{n}\right)$ vanishes unless

$$
\sum_{k=1}^{n} v_{\mathrm{a}_{i}}\left(\gamma_{k}\right)=\sum_{k=1}^{n} v_{\mathrm{b}_{i}}\left(\gamma_{k}\right), \quad i \in\{1,2\} .
$$

(ii) We have $\mathrm{F}_{g}^{A}\left(\alpha ; \gamma_{1}, \ldots, \gamma_{n}\right) \in \operatorname{QMod}_{2(g-2)+2 \ell}$ for $\ell=\sum_{k=1}^{n} v_{\mathrm{a}_{2}}\left(\gamma_{k}\right)$.

The cotangent line classes $\psi_{1}, \ldots, \psi_{n} \in H^{2}\left(\bar{M}_{g, n}(A, \beta), \mathbb{Q}\right)$ can be expressed as pull-backs of the corresponding cotangent line classes from $\vec{M}_{g, n}$ up to boundary corrections. ${ }^{6}$ Integration over the boundary corrections is governed by the splitting formula for reduced invariants. The boundary corrections yield integrals of lower genus or fewer marked points. Arguing inductively, the descendent series $\mathrm{F}_{g}^{A}\left(\tau_{a_{1}}\left(\gamma_{1}\right) \cdots \tau_{a_{n}}\left(\gamma_{n}\right)\right)$ can therefore be expressed in terms of the series

$$
\left\{\mathrm{F}_{g^{\prime}}^{A}\left(\alpha^{\prime} ; \gamma_{1}^{\prime}, \ldots, \gamma_{n^{\prime}}^{\prime}\right)\right\}
$$

for various $\alpha^{\prime}, g^{\prime}, \gamma_{1}^{\prime}, \ldots, \gamma_{n^{\prime}}^{\prime}$. By a simple verification, the splitting formula preserves the vanishing and modularity statements of Theorem 5.1. Hence, Proposition 5.2 implies Theorem 5.1.

The balancing condition of part (i) of Proposition 5.2 follows easily from a Hodge-theoretic argument. Alternatively, the balancing condition can be obtained inductively via the proof of part (ii) of Proposition 5.2.

${ }^{6}$ The unstable $g=0$ cases do not play a role. For abelian varieties, genus 0 invariants (standard or reduced) are non-vanishing only in the constant map case where stability requires three special points. 


\section{Curve Counting on ABElian SURFACES AND ThreEFoldS}

\subsection{Proof of Proposition 5.2(i)}

By [Tat65], or by a direct Mumford-Tate group calculation in case $E_{1}$ and $E_{2}$ are generic, the subring of Hodge classes on $A^{n}=\left(E_{1} \times E_{2}\right)^{n}$, denoted by $\operatorname{Hdg}^{*}\left(A^{n}\right) \subset H^{*}\left(A^{n}, \mathbb{Q}\right)$, is generated by divisor classes. (In particular, all Hodge classes on $A^{n}$ are algebraic.)

Hence, under the Künneth decomposition, the $\operatorname{ring} \operatorname{Hdg}^{*}\left(A^{n}\right)$ is generated by pull-backs of divisors classes in

$$
H^{2}\left(E_{i}, \mathbb{Q}\right), \quad H^{1}\left(E_{i}, \mathbb{Q}\right) \otimes H^{1}\left(E_{i}, \mathbb{Q}\right), \quad H^{1}\left(E_{1}, \mathbb{Q}\right) \otimes H^{1}\left(E_{2}, \mathbb{Q}\right),
$$

for $i=1,2$. We have $H^{2}\left(E_{i}, \mathbb{Q}\right)=\left\langle\omega_{i}\right\rangle=\left\langle\mathrm{a}_{i} \mathrm{~b}_{i}\right\rangle$. By the genericity of $E_{1}$ and $E_{2}$, all divisor classes in $H^{1}\left(E_{i}, \mathbb{Q}\right) \otimes H^{1}\left(E_{i}, \mathbb{Q}\right)$ are multiples of $\mathrm{a}_{i} \otimes \mathrm{b}_{i}-\mathrm{b}_{i} \otimes \mathrm{a}_{i}$, and there are no non-zero divisor classes in $H^{1}\left(E_{1}, \mathbb{Q}\right) \otimes H^{1}\left(E_{2}, \mathbb{Q}\right)$.

It follows that all classes in $\operatorname{Hdg}^{*}\left(A^{n}\right)$ are linear combinations of $\delta_{1} \otimes \cdots \otimes \delta_{n}$, where $\delta_{k} \in$ $H^{*}(A, \mathbb{Q})$ are monomial classes as defined in Section 5.1 such that

$$
\sum_{k=1}^{n} v_{\mathrm{a}_{i}}\left(\delta_{k}\right)=\sum_{k=1}^{n} v_{\mathrm{b}_{i}}\left(\delta_{k}\right), \quad i \in\{1,2\} .
$$

Consider the evaluation map ev: $\bar{M}_{g, n}(A, \beta) \rightarrow A^{n}$. By the push-pull formula, we have

$$
\left\langle\alpha ; \gamma_{1}, \ldots, \gamma_{n}\right\rangle_{g,(1, d)}^{A, \text { red }}=\int_{\mathrm{ev}_{*}\left(\pi^{*}(\alpha) \cap\left[\bar{M}_{g, n}(A, \beta)\right]^{\mathrm{red}}\right)} \gamma_{1} \otimes \cdots \otimes \gamma_{n} .
$$

By the algebraicity of $\alpha$ and $\left[\bar{M}_{g, n}(A, \beta)\right]^{\text {red }}$, the integral (5.1) is 0 unless

$$
\sum_{k=1}^{n} v_{\mathrm{a}_{i}}\left(\gamma_{k}\right)=\sum_{k=1}^{n} v_{\mathrm{b}_{i}}\left(\gamma_{k}\right), \quad i \in\{1,2\}
$$

\subsection{Proof of Proposition 5.2(ii): Base cases}

We argue by induction on the pair $(g, n)$, where $g \geqslant 1$ is the genus of the source curve and $n \geqslant 0$ is the number of marked points. We order the pairs $(g, n)$ lexicographically: we have $\left(g^{\prime}, n^{\prime}\right)<(g, n)$ if and only if

- $g^{\prime}<g$ or

- $g^{\prime}=g$ and $n^{\prime}<n$.

Base cases: $g=1$ and $n \geqslant 0$. We have already observed that Theorem 5.1 holds in all $g=1$ cases. Proposition 5.2 holds in $g=1$ by the same argument. We discuss the $n=0$ and $n=1$ cases as examples.

In case $(g, n)=(1,0)$, the only series is $\mathrm{F}_{1}^{A}(;)=0$ since the reduced virtual dimension is 1 and there are no insertions.

In case $(g, n)=(1,1)$, the moduli space $\bar{M}_{1,1}(A,(1, d))$ has reduced virtual dimension 2 . We must have either $\alpha \in R^{1}\left(\bar{M}_{1,1}\right)$ or $\tau(\mathrm{p})$ as integrands. Such an $\alpha$ is a multiple of the class of a point on $\bar{M}_{1,1}$. Because a generic elliptic curve does not admit a non-vanishing map to $E_{1} \times E_{2}$, the integral vanishes. In the second case, we evaluate

$$
\mathrm{F}_{1}^{A}(1 ; \mathrm{p})=\left\langle\tau_{0}(\mathrm{p})\right\rangle_{1,(1,0)}^{A, \text { red }}=1,
$$

which is a quasi-modular form of weight 0 . 


\section{J. Bryan, G. Oberdieck, R. Pandharipande And Q. Yin}

\subsection{Proof of Proposition 5.2(ii): Induction}

Consider a pair $(g, n)$ satisfying $g \geqslant 2$ and assume that Proposition 5.2 is proven in all cases $\left(g^{\prime}, n^{\prime}\right)$ where $\left(g^{\prime}, n^{\prime}\right)<(g, n)$.

Let $\gamma_{1}, \ldots, \gamma_{n} \in H^{*}(A, \mathbb{Q})$ be monomial classes, and let $\alpha \in R^{*}\left(\bar{M}_{g, n}\right)$ be a tautological class of pure degree. We must prove that Proposition 5.2 holds for the series

$$
\mathrm{F}_{g}^{A}\left(\alpha ; \gamma_{1}, \ldots, \gamma_{n}\right) \text {. }
$$

We may assume that the dimension constraint

$$
2 g+2 n=\operatorname{deg}(\alpha)+\sum_{i=1}^{n} \operatorname{deg}\left(\gamma_{i}\right)
$$

is satisfied, since the series (5.2) vanishes otherwise. In the dimension constraint (5.3), deg denotes the real cohomological degree of a class (on both $\bar{M}_{g, n}$ and $A$ ).

Case (i): $\sum_{i=1}^{n} \operatorname{deg}\left(\gamma_{i}\right) \leqslant 2 n$. From dimension constraint (5.3), we find

$$
\operatorname{deg}(\alpha)=2 g+2 n-\sum_{i} \operatorname{deg}\left(\gamma_{i}\right) \geqslant 2 g
$$

or, equivalently, $\alpha \in R^{\geqslant g}\left(\bar{M}_{g, n}\right)$. Using the strong form of Getzler-Ionel vanishing proven in [FP05, Proposition 2], we can find a class

$$
\widetilde{\alpha} \in R^{*}\left(\partial \bar{M}_{g, n}\right)
$$

such that $\iota_{*} \widetilde{\alpha}=\alpha$, where $\iota: \partial \bar{M}_{g, n} \rightarrow \bar{M}_{g, n}$ is the inclusion of the boundary. By the splitting formula, the series

$$
\mathrm{F}_{g}^{A}\left(\alpha ; \gamma_{1}, \ldots, \gamma_{n}\right)=\mathrm{F}_{g}^{A}\left(\iota_{*} \widetilde{\alpha} ; \gamma_{1}, \ldots, \gamma_{n}\right)
$$

is expressed in terms of a linear combination of series

$$
\mathrm{F}_{g^{\prime}}^{A}\left(\widetilde{\alpha} ; \widetilde{\gamma}_{1}, \ldots, \widetilde{\gamma}_{n^{\prime}}\right) \text { for which }\left(g^{\prime}, n^{\prime}\right)<(g, n) .
$$

The induction hypothesis for the latter implies that Proposition 5.2 holds for $\mathrm{F}_{g}^{A}\left(\alpha ; \gamma_{1}, \ldots, \gamma_{n}\right)$.

Case (ii): $\sum_{i=1}^{n} \operatorname{deg}\left(\gamma_{i}\right)>2 n$. Consider the moduli space $M_{g, n} \subset \bar{M}_{g, n}$ of non-singular genus $g \geqslant 2$ curves with $n$ marked points. The tautological ring $R^{*}\left(M_{g, n}\right)$ is generated by classes pulled back from $M_{g}$ via the forgetful map $p: M_{g, n} \rightarrow M_{g}$ and the cotangent line classes $\psi_{1}, \ldots, \psi_{n} \in H^{2}\left(M_{g, n}, \mathbb{Q}\right)$. A class $\alpha \in R^{*}\left(\bar{M}_{g, n}\right)$ can therefore be written as a sum of classes of the form

- $\iota_{*}(\widetilde{\alpha})$ for $\widetilde{\alpha} \in R^{*}\left(\partial \bar{M}_{g, n}\right)$,

- $\psi_{1}^{k_{1}} \cup \cdots \cup \psi_{n}^{k_{n}} \cup p^{*}(\zeta)$ for $k_{1}, \ldots, k_{n} \geqslant 0$ and $\zeta \in R^{*}\left(\bar{M}_{g}\right)$.

Here, we let $\psi_{1}, \ldots, \psi_{n}$ denote also the cotangent line classes on $\bar{M}_{g, n}$.

A summand of the form $\iota_{*}(\widetilde{\alpha})$ is expressed by the splitting formula in lower-order terms; see Case (i). Hence, we may assume

$$
\alpha=\psi_{1}^{k_{1}} \cup \cdots \cup \psi_{n}^{k_{n}} \cup p^{*}(\zeta) .
$$

Case (ii-a): There exists an $i$ for which $k_{i}>0$. We assume $k_{1}>0$. If $\operatorname{deg}\left(\gamma_{1}\right) \leqslant 3$, we first apply the vanishing of Lemma 4.2 for $\gamma_{1}$ and $\gamma=\gamma_{2} \otimes \cdots \otimes \gamma_{n}$. Using the abelian vanishing 


\section{CURVE COUNTING ON ABELIAN SURFACES AND THREEFOLDS}

relation (see also (4.3)), we find that $\mathrm{F}_{g}^{A}\left(\alpha ; \gamma_{1}, \gamma_{2}, \ldots, \gamma_{n}\right)$ can be expressed as a sum of series

$$
\pm \mathrm{F}_{g}^{A}\left(\alpha ; \gamma_{1} \cup \delta, \gamma_{2}^{\prime}, \ldots, \gamma_{n}^{\prime}\right)
$$

for various monomial classes $\delta, \gamma_{2}^{\prime}, \ldots, \gamma_{n}^{\prime} \in H^{*}(A, \mathbb{Q})$ with $\operatorname{deg}(\delta) \geqslant 1$.

The above relation increases the degree of $\gamma_{1}$. By induction on $\operatorname{deg}\left(\gamma_{1}\right)$, we may assume $\operatorname{deg}\left(\gamma_{1}\right)=4$ or, equivalently, $\gamma_{1}=\mathrm{p}$.

Next, we use the degeneration of $A=E_{1} \times E_{2}$ to the normal cone of an elliptic fiber $E=E_{2}$ of the projection to $E_{1}$,

$$
A \rightsquigarrow A \cup_{E}\left(\mathbb{P}^{1} \times E\right) .
$$

We choose the point class $\gamma_{1}$ to lie (after degeneration) on the component $\mathbb{P}^{1} \times E$. The distribution of the remaining classes $\gamma_{2}, \ldots, \gamma_{n}$ can be chosen freely.

For classes $\widetilde{\alpha} \in R^{*}\left(\bar{M}_{g, r}\right), \xi \in H^{*}(E, \mathbb{Q})$, and $\widetilde{\gamma}_{1}, \ldots, \widetilde{\gamma}_{r}$ on $A$ and $\mathbb{P}^{1} \times E$, respectively, we define

$$
\begin{aligned}
\mathrm{F}_{g}^{A / E}\left(\widetilde{\alpha} ; \widetilde{\gamma}_{1}, \ldots, \widetilde{\gamma}_{r} ; \xi\right) & =\sum_{d \geqslant 0}\left\langle\widetilde{\alpha} ; \prod_{i=1}^{r} \tau_{0}\left(\widetilde{\gamma}_{i}\right) \mid \xi\right\rangle_{g,(1, d)}^{A / E, \text { red }} q^{d}, \\
\mathrm{~F}_{g}^{\left(\mathbb{P}^{1} \times E\right) / E}\left(\widetilde{\alpha} ; \widetilde{\gamma}_{1}, \ldots, \widetilde{\gamma}_{r} ; \xi\right) & =\sum_{d \geqslant 0}\left\langle\widetilde{\alpha} ; \prod_{i=1}^{r} \tau_{0}\left(\widetilde{\gamma}_{i}\right) \mid \xi\right\rangle_{g,(1, d)}^{\left(\mathbb{P}^{1} \times E\right) / E} q^{d},
\end{aligned}
$$

where we use the bracket notation defined in Section 4.5.

For $\gamma_{1}=\mathrm{p}$, the degeneration formula then yields

$$
\mathrm{F}_{g}^{A}\left(\alpha ; \gamma_{1}, \ldots, \gamma_{n}\right)=\sum_{\left(g^{\prime}, n^{\prime}\right) \leqslant(g, n-1)} \mathrm{F}_{g^{\prime}, n^{\prime}}^{A / E}\left(\alpha^{\prime} ;(\ldots) ; \xi\right) \cdot \mathrm{F}_{g^{\prime \prime}, n^{\prime \prime}}^{\left(\mathbb{P}^{1} \times E\right) / E}\left(\alpha^{\prime \prime} ; \gamma_{1},(\ldots) ; \xi^{\vee}\right) .
$$

The summation here is over splittings

$$
g=g^{\prime}+g^{\prime \prime}, \quad n=n^{\prime}+n^{\prime \prime}
$$

and distributions $(\ldots)$ of the insertions $\gamma_{2}, \ldots, \gamma_{n}$. The marking numbers $n^{\prime}$ and $n^{\prime \prime}$ are placed in the subscripts of the generating series inside the sum for clarity. (The relative points are not included in the counts $n^{\prime}$ and $n^{\prime \prime}$.) The class $\alpha$ determines $\alpha^{\prime}$ and $\alpha^{\prime \prime}$ by restriction. Finally, there is also a sum over all relative conditions

$$
\xi \in\left\{1, \mathrm{a}_{2}, \mathrm{~b}_{2}, \omega_{2}\right\},
$$

where $\xi^{\vee}$ denotes the class dual to $\xi$.

Lemma 5.3. The primitive potential for $\left(\mathbb{P}^{1} \times E\right) / E$ satisfies the following properties for all $g^{\prime \prime} \geqslant 0$ :

(i) The series $\mathrm{F}_{g^{\prime \prime}}^{\left(\mathbb{P}^{1} \times E\right) / E}\left(\alpha^{\prime \prime} ; \widetilde{\gamma}_{1}, \ldots, \widetilde{\gamma}_{n^{\prime \prime}} ; \xi^{\vee}\right)$ vanishes unless

$$
v_{\mathrm{a}_{2}}\left(\xi^{\vee}\right)+\sum_{k=1}^{n^{\prime \prime}} v_{\mathrm{a}_{2}}\left(\widetilde{\gamma}_{k}\right)=v_{\mathrm{b}_{2}}\left(\xi^{\vee}\right)+\sum_{k=1}^{n^{\prime \prime}} v_{\mathrm{b}_{2}}\left(\widetilde{\gamma}_{k}\right) .
$$

(ii) We have $\mathrm{F}_{g^{\prime \prime}}^{\left(\mathbb{P}^{1} \times E\right) / E}\left(\alpha^{\prime \prime} ; \widetilde{\gamma}_{1}, \ldots, \widetilde{\gamma}_{n^{\prime \prime}} ; \xi^{\vee}\right) \in \operatorname{QMod}_{2 g^{\prime \prime}+2 \ell^{\prime \prime}-2}$ for

$$
\ell^{\prime \prime}=v_{\mathrm{a}_{2}}\left(\xi^{\vee}\right)+\sum_{k=1}^{n^{\prime \prime}} v_{\mathrm{a}_{2}}\left(\widetilde{\gamma}_{k}\right) \text {. }
$$




\section{J. Bryan, G. Oberdieck, R. Pandharipande and Q. Yin}

Proof. Because there is only algebraic cohomology on $\mathbb{P}^{1}$, the vanishing statement (i) follows from the fact that the virtual class is algebraic.

In [MP06], the relative invariants of $\left(\mathbb{P}^{1} \times E\right) / E$ were effectively expressed in terms of the absolute descendent invariants of $\mathbb{P}^{1} \times E$ through relations obtained by the following operations:

- degeneration of $\mathbb{P}^{1} \times E$ to $\mathbb{P}^{1} \times E \cup_{E} \mathbb{P}^{1} \times E$,

- localization on the $\mathbb{P}^{1}$-factor,

- rubber calculus.

A simple verification shows that the resulting relations respect modularity of the weight specified by part (ii) for each of these operations. Hence, we are reduced to the case of absolute descendent invariants of $\mathbb{P}^{1} \times E$.

Then, virtual localization on the $\mathbb{P}^{1}$-factor expresses the descendent invariants of $\mathbb{P}^{1} \times E$ in terms of the descendent invariants of E. Finally, [MPT10, Proposition 28] yields the required modularity property (ii).

For the last step, instead of localization, the product formula [Beh99] may be used to reduce $\mathbb{P}^{1} \times E$ to the case of the descendent invariants of the elliptic curve $E$ since the Gromov-Witten classes of $\mathbb{P}^{1}$ are known to be tautological [GP99].

Lemma 5.3 controls the factor on the right inside the sum of (5.5). Part (ii) of Lemma 5.3 is a refinement of [MPT10, Lemma 30]. However, the proof is the same as given in [MPT10].

The factor on the left inside the sum of (5.5) is more difficult to control. We will consider the terms of the sum corresponding to

$$
\left(g^{\prime}, n^{\prime}\right)<(g, n-1) \quad \text { and } \quad\left(g^{\prime}, n^{\prime}\right)=(g, n-1)
$$

separately. Lemma 5.4 below shows how to apply the inductive hypothesis to the terms in the sum (5.5) with $\left(g^{\prime}, n^{\prime}\right)<(g, n-1)$. The case $\left(g^{\prime}, n^{\prime}\right)=(g, n-1)$ will be considered afterward.

Lemma 5.4. Let $\left(g^{\prime}, n^{\prime}\right)<(g, n-1)$. The primitive potential for $A / E$ satisfies the following properties:

(i) The series $\mathrm{F}_{g^{\prime}}^{A / E}\left(\alpha^{\prime} ; \widetilde{\gamma}_{1}, \ldots, \widetilde{\gamma}_{n^{\prime}} ; \xi\right)$ vanishes unless

$$
v_{\mathrm{a}_{i}}(\xi)+\sum_{k=1}^{n^{\prime}} v_{\mathrm{a}_{i}}\left(\widetilde{\gamma}_{k}\right)=v_{\mathrm{b}_{i}}(\xi)+\sum_{k=1}^{n^{\prime}} v_{\mathrm{b}_{i}}\left(\widetilde{\gamma}_{k}\right), \quad i \in\{1,2\} .
$$

(ii) We have $\mathrm{F}_{g^{\prime}}^{A / E}\left(\alpha^{\prime} ; \widetilde{\gamma}_{1}, \ldots, \widetilde{\gamma}_{n^{\prime}} ; \xi\right) \in \operatorname{QMod}_{2\left(g^{\prime}-2\right)+2 \ell^{\prime}}$ for

$$
\ell^{\prime}=v_{\mathrm{a}_{2}}(\xi)+\sum_{k=1}^{n^{\prime}} v_{\mathrm{a}_{2}}\left(\widetilde{\gamma}_{k}\right)
$$

Proof. We apply the degeneration formula to

$$
\mathrm{F}_{g^{\prime}}^{A}\left(\alpha^{\prime} ; \widetilde{\gamma}_{1}, \ldots, \widetilde{\gamma}_{n^{\prime}}, \omega_{1} \xi\right)
$$

with the specialization to $\mathbb{P}^{1} \times E$ for the last point and the specialization by pull-back for the other insertions. Since $\left(g^{\prime}, n^{\prime}\right)<(g, n-1)$, we have $\left(g^{\prime}, n^{\prime}+1\right)<(g, n)$, so the induction hypothesis applies to the series (5.6). 


\section{Curve Counting on ABELIAN SuRfaces AND THREefolds}

The degeneration formula yields a relation involving the relative geometries $A / E$ and $\left(\mathbb{P}^{1} \times\right.$ $E) / E$,

$$
\mathrm{F}_{g^{\prime}}^{A}\left(\alpha^{\prime} ; \widetilde{\gamma}_{1}, \ldots, \widetilde{\gamma}_{n^{\prime}}, \omega_{1} \xi\right)=\sum_{\left(g_{\circ}^{\prime}, n_{\circ}^{\prime}\right) \leqslant\left(g^{\prime}, n^{\prime}\right)} \mathrm{F}_{g_{\circ}^{\prime}, n_{\circ}^{\prime}}^{A / E}\left(\alpha_{\circ}^{\prime} ;(\ldots) ; \widetilde{\xi}\right) \cdot \mathrm{F}_{g_{\bullet}^{\prime}, n_{\bullet}^{\prime}}^{\left(\mathbb{P}^{1} \times E\right) / E}\left(\alpha_{\bullet}^{\prime} ;(\ldots), \omega_{1} \xi ; \widetilde{\xi}^{\vee}\right)
$$

The summation here is over splittings

$$
g^{\prime}=g_{\circ}^{\prime}+g_{\bullet}^{\prime}, \quad n^{\prime}+1=n_{\circ}^{\prime}+n_{\bullet}^{\prime}
$$

and distributions $(\ldots)$ of the insertions $\widetilde{\gamma}_{1}, \ldots, \widetilde{\gamma}_{n^{\prime}}$. The class $\alpha^{\prime}$ determines $\alpha_{\circ}^{\prime}$ and $\alpha_{\bullet}^{\prime}$ by restriction. There is also a sum over all relative conditions $\widetilde{\xi} \in\left\{1, \mathrm{a}_{2}, \mathrm{~b}_{2}, \omega_{2}\right\}$.

We now analyze the $\left(g_{\circ}^{\prime}, n_{\circ}^{\prime}\right)=\left(g^{\prime}, n^{\prime}\right)$ term of the sum in $(5.7)$,

$$
\mathrm{F}_{g^{\prime}}^{A / E}\left(\alpha^{\prime} ; \widetilde{\gamma}_{1}, \ldots, \widetilde{\gamma}_{n^{\prime}} ; \widetilde{\xi}\right) \cdot \mathrm{F}_{0,1}^{\left(\mathbb{P}^{1} \times E\right) / E}\left(\omega_{1} \xi ; \widetilde{\xi}^{\vee}\right)
$$

Since genus 0 stable maps do not represent classes of type $(1, d)$ with $d>0$ on $\mathbb{P}^{1} \times E$, we have

$$
\mathrm{F}_{0,1}^{\left(\mathbb{P}^{1} \times E\right) / E}\left(\omega_{1} \xi ; \widetilde{\xi}^{\vee}\right)=\left\langle\widetilde{\xi}^{\vee} \mid \tau_{0}\left(\omega_{1} \xi\right)\right\rangle_{0,(1,0)}^{\left(\mathbb{P}^{1} \times E\right) / E}=\delta_{\xi, \widetilde{\xi}}
$$

The second equality is obtained by the identification of the moduli space of maps by the location of the relative point,

$$
\bar{M}_{0,0}\left(\left(\mathbb{P}^{1} \times E\right) / E,(1,0)\right) \cong E .
$$

Combining the above, we can rewrite (5.8) as simply

$$
\mathrm{F}_{g^{\prime}}^{A / E}\left(\alpha^{\prime} ; \widetilde{\gamma}_{1}, \ldots, \widetilde{\gamma}_{n^{\prime}} ; \xi\right) \text {. }
$$

We find that the series $\mathrm{F}^{A}$ for the absolute geometry can be expressed in terms of the series $\mathrm{F}^{A / E}$ for the relative geometry by a transformation matrix $\mathrm{M}$ which is upper triangular with entries 1 on the diagonal. By Lemma 5.3, the off-diagonal terms of $\mathrm{M}$ are given by quasi-modular forms. By inverting the unipotent matrix $M$ and applying the induction hypothesis to $\mathrm{F}^{A}$, we find that the relative invariants $\mathrm{F}^{A / E}$ are quasi-modular forms.

The weight and vanishing statement can now be deduced from a careful consideration of the entries of $\mathrm{M}^{-1}$. Alternatively, we may argue via a (second) induction on $\left(g^{\prime}, n^{\prime}\right)$. In case $\left(g^{\prime}, n^{\prime}\right)=(1,1)$, there are no lower-order terms in $(5.7)$, and we are done. If the statement is true for all $\left(g_{\circ}^{\prime}, n_{\circ}^{\prime}\right)<\left(g^{\prime}, n^{\prime}\right)$, then the statement follows directly from (5.7) and the induction hypothesis.

We now turn to the $\left(g^{\prime}, n^{\prime}\right)=(g, n-1)$ term in the sum $(5.5)$ :

$$
\mathrm{F}_{g, n-1}^{A / E}\left(\alpha ; \gamma_{2}, \ldots, \gamma_{n} ; \omega\right) \cdot \mathrm{F}_{0,1}^{\left.\mathbb{P}^{1} \times E\right) / E}\left(\gamma_{1} ; 1\right)
$$

As we have seen above, only curves in class $(1,0)$ contribute to the series $\mathrm{F}_{0,1}^{\left(\mathbb{P}^{1} \times E\right) / E}\left(\gamma_{1} ; 1\right)$. By the identification of the moduli space (5.9), we have

$$
\mathrm{F}_{0,1}^{\left(\mathbb{P}^{1} \times E\right) / E}\left(\gamma_{1} ; 1\right)=\left\langle 1 \mid \tau_{0}(\mathrm{p})\right\rangle_{0,(1,0)}^{\left(\mathbb{P}^{1} \times E\right) / E}=1 .
$$

Hence, the $\left(g^{\prime}, n^{\prime}\right)=(g, n-1)$ term is

$$
\mathrm{F}_{g, n-1}^{A / E}\left(\alpha ; \gamma_{2}, \ldots, \gamma_{n} ; \omega\right)
$$

where the class $\alpha \in R^{*}\left(\bar{M}_{g, n}\right)$ is pulled back to $\bar{M}_{g, n-1}(A / E,(1, d))$ via the map

$$
\pi: \bar{M}_{g, n-1}(A / E,(1, d)) \rightarrow \bar{M}_{g, n},
$$




\section{J. Bryan, G. Oberdieck, R. Pandharipande And Q. Yin}

which takes the relative point on the left to the marking 1 on the right. We must prove that the induction hypothesis implies that

(i) the potential $\mathrm{F}_{g, n-1}^{A / E}\left(\alpha ; \gamma_{2}, \ldots, \gamma_{n} ; \omega\right)$ vanishes unless

$$
v_{\mathrm{a}_{i}}(\xi)+\sum_{k=2}^{n} v_{\mathrm{a}_{i}}\left(\gamma_{k}\right)=v_{\mathrm{b}_{i}}(\xi)+\sum_{k=2}^{n} v_{\mathrm{b}_{i}}\left(\gamma_{k}\right), \quad i \in\{1,2\} ;
$$

(ii) we have $\mathrm{F}_{g, n-1}^{A / E}\left(\alpha ; \gamma_{2}, \ldots, \gamma_{n} ; \omega\right) \in \operatorname{QMod}_{2(g-2)+2 \ell}$ for

$$
\ell=v_{\mathrm{a}_{2}}(\xi)+\sum_{k=2}^{n} v_{\mathrm{a}_{2}}\left(\gamma_{k}\right) .
$$

We proceed by studying the cotangent lines. Let

$$
L_{\mathrm{rel}} \rightarrow \bar{M}_{g, n-1}(A / E,(1, d)), \quad L_{1} \rightarrow \bar{M}_{g, n}
$$

denote the respective cotangent lines at the relative point and the first marking.

Lemma 5.5. After pull-back via $\pi$, we have an isomorphism $L_{\mathrm{rel}} \cong \pi^{*} L_{1}$ on $\bar{M}_{g, n-1}(A / E,(1, d))$.

Proof. Let $C$ be the $n$-pointed domain of a map $f: C \rightarrow \widetilde{A}$ parameterized by the moduli space $\bar{M}_{g, n-1}(A / E,(1, d))$. The $n$ points consist of the relative point together with the $n-1$ standard markings. The target $\widetilde{A}$ is a possible accordian destabilization of $A$ along $E$. The lemma is a consequence of the following claim: the n-pointed curve $C$ is Deligne-Mumford stable.

Since $g \geqslant 1$ and $n \geqslant 1$, to prove the stability of the $n$-pointed curve $C$, we need only consider the non-singular rational components $P \subset C$ :

- If $f(P) \subset A$, then $f$ is constant on $P$, that is, $f(P) \in A \backslash E$, and $P$ must carry at least three special points by the definition of map stability.

- If $f(P)$ is contained in a rubber bubble over $E$, then $P$ is mapped to a point of $E$ and therefore must map to a fiber of the bubble. Stability of the bubble then requires the existence of at least three special points of $P$.

Since the $n$-pointed curve $C$ is stable, there is no contraction of components associated with the map $\pi$. Hence, the cotangent lines are isomorphic.

The relative divisor $E \subset A$ is the fiber over a point $0_{E_{1}} \in E_{1}$. Let $f: C \rightarrow \widetilde{A}$ be a stable map parameterized by the moduli space $\bar{M}_{g, n-1}(A / E,(1, d))$, and let $p_{\text {rel }} \in C$ be the relative point. Composition with the canonical projections $\epsilon: \widetilde{A} \rightarrow A \rightarrow E_{1}$ yields a map

$$
\epsilon f: C \rightarrow E_{1} \quad \text { with } \quad \epsilon f\left(p_{\text {rel }}\right)=0_{E_{1}} .
$$

The cotangent line $L_{\text {rel }}$ carries a canonical section via the differential of $\epsilon f$,

$$
s: \mathbb{C}=T_{0_{E_{1}}}^{*}\left(E_{1}\right) \rightarrow L_{\mathrm{rel}} .
$$

The vanishing locus ${ }^{7}$ of $s$ is the boundary of the moduli space $\bar{M}_{g, n-1}(A / E,(1, d))$ corresponding to the first bubble over $E$.

Since $\alpha$ is of the form (5.4) with $k_{1}>0$, a factor $\psi_{1}=c_{1}\left(L_{1}\right)$ can be extracted from $\alpha$, giving $\alpha=\psi_{1} \cdot \widetilde{\alpha}$. After pull-back via $\pi$, we have $\pi^{*}\left(\psi_{1}\right)=c_{1}\left(L_{\text {rel }}\right)$ by Lemma 5.5 . Via the vanishing

\footnotetext{
${ }^{7}$ The geometry is pulled back from the Artin stack of degenerations of $A / E$.
} 


\section{CuRve COUNTING ON ABELIAN SURFACES AND THREEFOLDS}

locus of the section $s$, we obtain the following equation for the series (5.10):

$$
\mathrm{F}_{g, n-1}^{A / E}\left(\alpha ; \gamma_{2}, \ldots, \gamma_{n} ; \omega\right)=\sum_{\left(g^{\prime}, n^{\prime}\right)<(g, n-1)} \mathrm{F}_{g^{\prime}, n^{\prime}}^{A / E}\left(\widetilde{\alpha}^{\prime} ;(\ldots) ; \xi\right) \cdot \mathrm{F}_{g^{\prime \prime}, n^{\prime \prime}}^{\mathrm{Rub}\left(\mathbb{P}^{1} \times E\right)}\left(\widetilde{\alpha}^{\prime \prime} ;(\ldots) ; \xi^{\vee}, \omega\right) \text {. }
$$

The summation here is over splittings

$$
g=g^{\prime}+g^{\prime \prime}, \quad n-1=n^{\prime}+n^{\prime \prime}
$$

and distributions $(\ldots)$ of the insertions $\gamma_{2}, \ldots, \gamma_{n}$. Only insertions $\gamma_{i}$ satisfying

$$
v_{\mathrm{a}_{1}}\left(\gamma_{i}\right)=v_{\mathrm{b}_{1}}\left(\gamma_{i}\right)=0
$$

can be distributed to the rubber series

$$
\mathrm{F}_{g^{\prime \prime}, n^{\prime \prime}}^{\mathrm{Rub}\left(\mathbb{P}^{1} \times E\right)}\left(\widetilde{\alpha}^{\prime \prime} ;(\ldots) ; \xi^{\vee}, \omega\right)=\sum_{d \geqslant 0}\left\langle\xi^{\vee}\left|\widetilde{\alpha}^{\prime \prime} ;(\ldots)\right| \omega\right\rangle_{g^{\prime \prime},(1, d)}^{\mathrm{Rub}\left(\mathbb{P}^{1} \times E\right)} q^{d} .
$$

By stability of the rubber, either $g^{\prime \prime}>0$ or $n^{\prime \prime}>0$. The class $\widetilde{\alpha}$ determines $\widetilde{\alpha}^{\prime}$ and $\widetilde{\alpha}^{\prime \prime}$ by restriction. Finally, there is also a sum on the right side of (5.11) over relative conditions $\xi$.

In the sum on the right side of (5.11), the balancing and modularity of the first factor

$$
\mathrm{F}_{g^{\prime}, n^{\prime}}^{A / E}\left(\widetilde{\alpha}^{\prime} ;(\ldots) ; \xi\right)
$$

is obtained from Lemma 5.4. The balancing and modularity of the rubber factor

$$
\mathrm{F}_{g^{\prime \prime}, n^{\prime \prime}}^{\operatorname{Rub}\left(\mathbb{P}^{1} \times E\right)}\left(\widetilde{\alpha}^{\prime \prime} ;(\ldots) ; \xi^{\vee}, \omega\right)
$$

follows from the rubber calculus and an argument parallel to the proof of Lemma 5.3. (We leave the details here to the reader.) The results together imply the required balancing and modularity for the series (5.10).

We now control the balancing and modularity of all terms in the sum on the right of (5.5). As a consequence, Proposition 5.2 holds for $\mathrm{F}_{g}^{A}\left(\alpha ; \gamma_{1}, \ldots, \gamma_{n}\right)$. The proof of the induction step for Case (ii-a) is complete.

Case (ii-b): $\alpha=p^{*}(\zeta)$ for some $\zeta \in R^{*}\left(\bar{M}_{g}\right)$. We may assume that $\gamma_{1}$ is of minimal degree:

$$
\operatorname{deg}\left(\gamma_{1}\right) \leqslant \operatorname{deg}\left(\gamma_{i}\right) \text { for all } i \in\{2, \ldots, n\} .
$$

Below, we will distinguish several subcases depending upon $\operatorname{deg}\left(\gamma_{1}\right)$.

Consider the map $\bar{M}_{g, n}(A,(1, d)) \rightarrow \bar{M}_{g, n-1}(A,(1, d))$ forgetting the first marking. The coefficients of the series

$$
\mathrm{F}_{g}^{A}\left(p^{*}(\zeta) ; \gamma_{1}, \ldots, \gamma_{n}\right)
$$

are integrals where all classes in the integrand, except for $\operatorname{ev}_{1}^{*}\left(\gamma_{1}\right)$, are pull-backs via the map forgetting the first marking.

Case $\operatorname{deg}\left(\gamma_{1}\right) \leqslant 1$. The series (5.12) vanishes by the push-pull formula since the fiber of the forgetful map has (complex) dimension 1.

Case $\operatorname{deg}\left(\gamma_{1}\right)=2$. We use the divisor equation for $\gamma_{1}$ and find

$$
\mathrm{F}_{g}^{A}\left(p^{*}(\zeta) ; \gamma_{1}, \ldots, \gamma_{n}\right)= \begin{cases}\mathrm{F}_{g}^{A}\left(p^{*}(\zeta) ; \gamma_{2}, \ldots, \gamma_{n}\right) & \text { if } \gamma_{1}=\mathrm{a}_{1} \mathrm{~b}_{1} \\ q \frac{d}{d q} \mathrm{~F}_{g}^{A}\left(p^{*}(\zeta) ; \gamma_{2}, \ldots, \gamma_{n}\right) & \text { if } \gamma_{1}=\mathrm{a}_{2} \mathrm{~b}_{2} \\ 0 & \text { otherwise }\end{cases}
$$




\section{J. Bryan, G. Oberdieck, R. Pandharipande And Q. Yin}

Because the differential operator

$$
q \frac{d}{d q}=\frac{1}{2 \pi i} \frac{\partial}{\partial \tau}
$$

preserves $\mathrm{QMod}_{*}$ and is homogeneous of degree 2, Proposition 5.2 holds for (5.12) by the induction hypothesis.

Case $\operatorname{deg}\left(\gamma_{1}\right)=3$. Since $\sum_{i} \operatorname{deg}\left(\gamma_{i}\right)$ is even, we must have $n \geqslant 2$. We order the classes $\gamma_{2}, \ldots, \gamma_{n}$ so that $\gamma_{2}, \ldots, \gamma_{k}$ are point classes and $\gamma_{k+1}, \ldots, \gamma_{n}$ are classes of degree 3 , for some $1 \leqslant k<n$.

We will use the abelian vanishing of Lemma 4.2 for $\gamma_{1}$ and $\gamma=\gamma_{2} \otimes \cdots \otimes \gamma_{n}$. Let $s \in$ $\left\{\mathrm{a}_{1}, \mathrm{~b}_{1}, \mathrm{a}_{2}, \mathrm{~b}_{2}\right\}$ be the factor with $v_{s}\left(\gamma_{1}\right)=0$. Using the abelian vanishing relation, we find

$$
\sum_{i=1}^{k} \mathrm{~F}_{g}^{A}(p^{*}(\zeta) ; \underbrace{\mathrm{p}, \ldots, \mathrm{p}}_{i-1}, \gamma_{1}, \underbrace{\mathrm{p}, \ldots, \mathrm{p}}_{k-i}, \gamma_{k+1}, \ldots, \gamma_{n})=\sum_{\substack{i=k+1 \\ v_{s}\left(\gamma_{i}\right)=1}}^{n} \pm \mathrm{F}_{g}^{A}\left(p^{*}(\zeta) ; \mathrm{p}, \gamma_{2}, \ldots, \widetilde{\gamma}_{i}, \ldots, \gamma_{n}\right),
$$

where $\widetilde{\gamma}_{i}=\gamma_{i} / s$ denotes the class $\gamma_{i}$ with the factor $s$ removed. The plus signs in the terms on the left-hand side require a careful accounting of the signs. Since the class $p^{*}(\zeta)$ is symmetric with respect to interchanging markings, the above equation simplifies to

$$
k \cdot \mathrm{F}_{g}^{A}\left(p^{*}(\zeta) ; \gamma_{1}, \ldots, \gamma_{n}\right)=\sum_{\substack{i=k+1 \\ v_{s}\left(\gamma_{i}\right)=1}}^{n} \pm \mathrm{F}_{g}^{A}\left(p^{*}(\zeta) ; \mathrm{p}, \gamma_{2}, \ldots, \widetilde{\gamma}_{i}, \ldots, \gamma_{n}\right) .
$$

Since $\widetilde{\gamma}_{i}$ is of degree 2, we may apply the divisor equation to each summand on the right side. As a result, the right side is reduced to terms of lower order; see Case $\operatorname{deg}\left(\gamma_{1}\right)=2$ above. By the induction hypothesis, Proposition 5.2 holds for (5.12).

Case $\operatorname{deg}\left(\gamma_{1}\right)=4$. All the insertions $\gamma_{1}, \ldots, \gamma_{n}$ must be point classes. If $n=1$, the dimension constraint (5.3) implies $\operatorname{deg}(\alpha)=2 g-2$ and hence $\zeta \in R^{g-1}\left(\bar{M}_{g}\right)$. By the strong form of Looijenga's vanishing $R^{\geqslant g-1}\left(M_{g}\right)=0$ proven in [FP05, Proposition 2], there exists a class $\widetilde{\zeta} \in$ $R^{*}\left(\partial \bar{M}_{g}\right)$ such that $\iota_{*} \widetilde{\zeta}=\zeta$. The pull-back $p^{*}(\zeta)$ via $p: \bar{M}_{g, n} \rightarrow \bar{M}_{g}$ can be written as the push-forward of a tautological class on the boundary $\partial \bar{M}_{g, n}$. Proposition 5.2 holds for (5.12) by the splitting formula and the induction hypothesis; see Case (i).

If $n \geqslant 2$, we use the degeneration $A \rightsquigarrow A \cup_{E}\left(\mathbb{P}^{1} \times E\right)$, which already appeared in Case (ii-a) above. We choose the point classes $\gamma_{1}$ and $\gamma_{2}$ to lie on the component $\mathbb{P}^{1} \times E$ after degeneration.

The degeneration formula then yields

$$
\mathrm{F}_{g}^{A}\left(\alpha ; \gamma_{1}, \ldots, \gamma_{n}\right)=\sum_{\left(g^{\prime}, n^{\prime}\right) \leqslant(g, n)} \mathrm{F}_{g^{\prime}, n^{\prime}}^{A / E}\left(\alpha^{\prime} ;(\ldots) ; \xi\right) \cdot \mathrm{F}_{g^{\prime \prime}, n^{\prime \prime}}^{\left(\mathbb{P}^{1} \times E\right) / E}\left(\alpha^{\prime \prime} ; \gamma_{1}, \gamma_{2},(\ldots) ; \xi^{\vee}\right),
$$

where the sum is as in $(5.5)$.

If $g^{\prime}=g$ in the sum (5.13), then the second factor is

$$
\mathrm{F}_{0, n^{\prime \prime}}^{\left(\mathbb{P}^{1} \times E\right) / E}\left(\alpha^{\prime \prime} ; \gamma_{1}, \gamma_{2},(\ldots) ; \xi^{\vee}\right) \text {. }
$$

Genus 0 stable maps do not represent classes of type $(1, d)$ with $d>0$ on $\mathbb{P}^{1} \times E$; hence only the curve class $(1,0)$ need be considered. Since there are no curves of type $(1,0)$ through two general points of $\mathbb{P}^{1} \times E$, the factor (5.14) vanishes. As a result, only $g^{\prime}<g$ terms appear in the sum (5.13). Proposition 5.2 holds for (5.12) by Lemmas 5.3 and 5.4. 


\section{CURVE COUNTING ON ABELIAN SURFACES AND THREEFOLDS}

The proof of the induction step has now been established in all cases. The proof of Proposition 5.2 is complete.

\section{$5.6 K 3$ surfaces}

Theorem 1.3 for abelian surfaces is exactly parallel to the modularity results [MPT10, Theorem 4 and Proposition 29] for the primitive descendent potential for $K 3$ surfaces. Though the argument for abelian surfaces is more difficult because of the presence of odd cohomology, several aspects are similar.

The refined modularity of Theorem 5.1 for abelian surfaces is strictly stronger than the statements of [MPT10] for $K 3$ surfaces. In fact, the proof of [MPT10] also yields the parallel refined statement for $K 3$ surfaces. The crucial point is to use the refined modularity of Lemma 5.3(ii) instead of the weaker modularity of [MPT10, Lemma 30]. We state the refined modularity for $K 3$ surfaces below following the notation of [MPT10].

Let $S \rightarrow \mathbb{P}^{1}$ be a non-singular, projective, elliptically fibered $K 3$ surface with a section. Let $\mathbf{s}, \mathbf{f} \in \mathrm{H}_{2}(S, \mathbb{Z})$ be the section and fiber class, respectively. The primitive descendent potential for the reduced Gromov-Witten theory of $S$ is defined by

$$
\mathrm{F}_{g}^{S}\left(\tau_{a_{1}}\left(\gamma_{1}\right) \cdots \tau_{a_{n}}\left(\gamma_{n}\right)\right)=\sum_{d \geqslant 0}\left\langle\tau_{a_{1}}\left(\gamma_{1}\right) \cdots \tau_{a_{n}}\left(\gamma_{n}\right)\right\rangle_{g, \mathbf{s}+d \mathbf{f}}^{S, \text { red }} q^{d-1}
$$

for $g \geqslant 0$.

We define a new degree function $\underline{\operatorname{deg}}(\gamma)$ for classes $\gamma \in H^{*}(S, \mathbb{Q})$ by the following rules:

- $\gamma \in H^{0}(S, \mathbb{Q}) \mapsto \underline{\operatorname{deg}}(\gamma)=0$,

- $\gamma \in H^{4}(S, \mathbb{Q}) \mapsto \overline{\mathrm{deg}}(\gamma)=2$.

For classes $\gamma \in H^{2}(S, \mathbb{Q})$, the degree is more subtle. Viewing the section and fiber classes also as elements of cohomology, we define

$$
V=\mathbb{Q} \mathbf{s} \oplus \mathbb{Q} f \subset H^{2}(S, \mathbb{Q}) .
$$

We have a direct sum decomposition

$$
\mathbb{Q} f \oplus V^{\perp} \oplus \mathbb{Q}(\mathrm{s}+\mathrm{f}) \cong H^{2}(S, \mathbb{Q}),
$$

where $V^{\perp}$ is defined with respect to the intersection form. We consider only classes $\gamma \in H^{2}(S, \mathbb{Q})$ which are pure with respect to the decomposition (5.15). Then,

- $\gamma \in \mathbb{Q} f \mapsto \underline{\operatorname{deg}}(\gamma)=0$,

- $\gamma \in V^{\perp} \mapsto \underline{\operatorname{deg}}(\gamma)=1$,

- $\gamma \in \mathbb{Q}(\mathrm{s}+\mathrm{f}) \mapsto \underline{\operatorname{deg}}(\gamma)=2$.

The modularity of [MPT10, Theorem 4 and Proposition 29] is refined by the following result.

Theorem 5.6. For deg-homogeneous classes $\gamma_{i} \in H^{*}(S, \mathbb{Q})$, we have

$$
\mathrm{F}_{g}^{S}\left(\tau_{a_{1}}\left(\gamma_{1}\right) \cdots \tau_{a_{n}}\left(\gamma_{n}\right)\right) \in \frac{1}{\Delta(q)} \mathrm{QMod}_{\ell}
$$

for $\ell=2 g+\sum_{i=1}^{n} \underline{\operatorname{deg}}\left(\gamma_{i}\right)$.

The discriminant modular form entering in Theorem 5.6 is

$$
\Delta(q)=q \prod_{n=1}^{\infty}\left(1-q^{n}\right)^{24}
$$




\section{J. Bryan, G. Oberdieck, R. Pandharipande And Q. Yin}

\section{Hyperelliptic curves}

\subsection{Overview}

The correspondence between hyperelliptic curves on a surface $S$ and rational curves in $\operatorname{Hilb}^{2}(S)$ has been used often to enumerate hyperelliptic curves on $S$; see [Gra01] for $S=\mathbb{P}^{2}$ and [Pon07] for $S=\mathbb{P}^{1} \times \mathbb{P}^{1}$. The main difficulty in applying the correspondence is the need of a non-degeneracy result concerning curves in $\mathbb{P}^{1} \times S$. For abelian surfaces, the required non-degeneracy, stated as $(\dagger)$ in Section 1.2.4, is expected to hold generically but is not known in most cases. The above correspondence then yields only conditional or virtual results on the number of hyperelliptic curves on an abelian surface, as pursued, for example, by Rose in [Ros14].

We proceed with our study of hyperelliptic curves in three steps. In Section 6.2, we provide several equivalent descriptions of $(\dagger)$ and a proof in genus 2 for a generic abelian surface. In Section 6.3, we prove an unconditional formula for the first non-trivial case of genus 3 hyperelliptic curves via explicit Gromov-Witten integrals, a boundary analysis, and the genus 2 result proven in Section 6.2.

In Section 6.4, we assume the existence of abelian surfaces $A$ and irreducible curve classes $\beta$ satisfying property $(\dagger)$ in all genera. Employing the correspondence above, we find a closed

formula for the $\mathrm{h}_{g, \beta}^{A, \mathrm{FLS}}$. While a similar strategy has been used in [Ros14] assuming the crepant resolution conjecture, our closed formula is new. Together with the strong modularity result of Theorem 5.1, we obtain a formula for the Gromov-Witten numbers $\mathrm{H}_{g,(1, d)}^{\mathrm{FLS}}$ which agrees with the genus 3 counts.

\subsection{Non-degeneracy for abelian surfaces}

We briefly recall the correspondences between hyperelliptic curves in $S$, curves in $\mathbb{P}^{1} \times S$, and rational curves in $\operatorname{Hilb}^{2}(S)$. For simplicity, we restrict to the case of abelian surfaces $S=A$; see [FKP09, Gra01, Obe18] for the general case.

Let $A$ be an abelian surface, and let $f: C \rightarrow A$ be a map from a non-singular hyperelliptic curve. Let $p: C \rightarrow \mathbb{P}^{1}$ be the double cover. Since $A$ contains no rational curves, $f$ does not factor through $p$. Consider the map $(p, f): C \rightarrow \mathbb{P}^{1} \times A$. The image $\bar{C}=\operatorname{Im}(C) \subset \mathbb{P}^{1} \times A$ is an irreducible curve, (flat) of degree 2 over $\mathbb{P}^{1}$, and has normalization $C \rightarrow \bar{C}$.

Let $\operatorname{Hilb}^{2}(A)$ be the Hilbert scheme of 2 points of $A$, and let $\Delta \subset \operatorname{Hilb}^{2}(A)$ denote the subvariety parameterizing non-reduced length 2 subschemes of $A$. By the universal property of the Hilbert scheme, the curve $\bar{C}$ induces a map $\phi: \mathbb{P}^{1} \rightarrow \operatorname{Hilb}^{2}(A)$ such that the image is not contained in $\Delta$.

Conversely, let $\phi: \mathbb{P}^{1} \rightarrow \operatorname{Hilb}^{2}(A)$ be a map whose image is not contained in $\Delta$. Since $A$ contains no rational curves, by pulling back the universal family $Z \subset \operatorname{Hilb}^{2}(A) \times A$, we obtain an irreducible curve $\bar{C} \subset \mathbb{P}^{1} \times A$ of degree 2 over $\mathbb{P}^{1}$. The normalization $C \rightarrow \bar{C}$ is hyperelliptic and induces a map $f: C \rightarrow A$.

Hence, there are bijective correspondences between

- maps $f: C \rightarrow A$ from non-singular hyperelliptic curves,

- irreducible curves $\bar{C} \subset \mathbb{P}^{1} \times A$ of degree 2 over $\mathbb{P}^{1}$,

- maps $\phi: \mathbb{P}^{1} \rightarrow \operatorname{Hilb}^{2}(A)$ with image not contained in $\Delta$.

The correspondences allow us to reformulate the non-degeneracy property $(\dagger)$.

LEMma 6.1 (Graber [Gra01]). Under the correspondences above, the following are equivalent: 


\section{CURVE COUNTING ON ABELIAN SURFACES AND THREEFOLDS}

(i) The differential of $f$ is injective at the Weierstrass points of $C$, and no conjugate nonWeierstrass points are mapped to the same point on $A$.

(ii) The curve $\bar{C}$ is non-singular.

(iii) The map $\phi$ meets $\Delta$ transversally.

Using a recent result of Poonen and Stoll [PS14], we verify that property $(\dagger)$ holds generically in genus 2 .

Lemma 6.2. Let $A$ be a generic abelian surface with a curve class $\beta$ of type $(1, d)$, and let $f: C \rightarrow A$ be a map from a non-singular genus 2 curve in class $\beta$. Then $f: C \rightarrow A$ satisfies condition (i) of Lemma 6.1.

Proof. The condition on the differential of $f$ is automatically satisfied by [LS02b, Proposition 2.2] for genus 2 curves on abelian surfaces.

Now, suppose that there exist a non-singular genus 2 curve $C$, two conjugate non-Weierstrass points $x, y \in C$, and a map $f: C \rightarrow A$ in class $\beta$ such that $f(x)=f(y)$. Up to translation, we may assume that $f$ maps a Weierstrass point $q \in C$ to $0_{A} \in A$. Then $f$ factors as

$$
C \stackrel{\text { aj }}{\rightarrow} J \stackrel{\pi}{\rightarrow} A,
$$

where $J$ is the Jacobian of $C$ and aj is the Abel-Jacobi map with respect to $q$. The hyperelliptic involution of $C$ corresponds to the automorphisms -1 of $J$ and $A$. For $x, y$ conjugate, this implies that $f(x)=f(y)$ is a 2-torsion point on $A$.

Since $C$ is of genus 2 and $\beta$ is of type $(1, d)$, the map $\pi$ is an isogeny of degree $d$. It follows that $\operatorname{both} \operatorname{aj}(x)$ and $\operatorname{aj}(y)$ are $2 d$-torsions on $J$.

In genus 2, the assumption that $A$ is generic implies that $C$ is generic. However, by [PS14, Theorem 7.1], a generic (Weierstrass-pointed) hyperelliptic curve $C$ meets the torsions of $J$ only at the Weierstrass points. Hence the points $x, y$ do not exist.

The proof of Lemma 6.2 works for any type $\left(d_{1}, d_{2}\right)$ with $d_{1}, d_{2}>0$. However, since multiple covered curves may arise, statement $(\dagger)$ is false for imprimitive classes in higher genus. The most basic counterexample is constructed by taking an étale double cover $C_{3} \rightarrow C_{2}$ of a non-singular genus 2 curve $C_{2} \subset A$. Then, $C_{3}$ is non-singular and hyperelliptic of genus 3 , but $C_{2}$ contains a Weierstrass point whose preimage in $C_{3}$ is a pair of conjugate non-Weierstrass points.

Furthermore, by the proof of [KLCM17, Theorem 1.6], for generic $A$ and $\beta$ of type $(1, d)$, property $(\dagger)$ also holds in the maximal geometric genus $g_{d}$. The value of $g_{d}$ is determined by the inequality (1.5). It is also shown that for every $g \in\left\{2, \ldots, g_{d}\right\}$, there exists at least one non-singular genus $g$ curve in $\mathbb{P}^{1} \times A$ of class $(2, \beta)$.

\subsection{Genus 3 hyperelliptic counts}

We prove here Proposition 1.4. We proceed in two steps. First, we evaluate $\mathrm{H}_{3,(1, d)}^{\mathrm{FLS}}$. Then, we identify the contributions from the boundary of the moduli space.

Lemma 6.3. For all $d \geqslant 1$, we have $\mathrm{H}_{3,(1, d)}^{\mathrm{FLS}}=d^{2} \sum_{m \mid d}\left(3 m^{2}-4 d m\right) / 4$.

Proof. On $\bar{M}_{3}$, let $\lambda_{1}$ be the first Chern class of the Hodge bundle, let $\delta_{0}$ be the class of the curves with a nonseparating node, and let $\delta_{1}$ be the class of curves with a separating node. By [HM82], we have

$$
\mathcal{H}_{3}=9 \lambda_{1}-\delta_{0}-3 \delta_{1}
$$




\section{J. Bryan, G. Oberdieck, R. Pandharipande And Q. Yin}

The lemma will be proven by the following three evaluations:

$$
\left\langle\lambda_{1}\right\rangle_{3,(1, d)}^{A, \mathrm{FLS}}=\frac{d^{2}}{12} \sum_{m \mid d} m^{3}, \quad\left\langle\delta_{0}\right\rangle_{3,(1, d)}^{A, \mathrm{FLS}}=d^{3} \sum_{m \mid d} m, \quad\left\langle\delta_{1}\right\rangle_{3,(1, d)}^{A, \mathrm{FLS}}=0 .
$$

The first equation follows directly from Theorem 1.1. For the second, we have

$$
\left\langle\delta_{0}\right\rangle_{3,(1, d)}^{A, \mathrm{FLS}}=\frac{1}{2}\left\langle\tau_{0}(\Delta)\right\rangle_{2,(1, d)}^{A, \mathrm{FLS}}=\frac{1}{2} \cdot 2 d \cdot\langle 1\rangle_{2,(1, d)}^{A, \mathrm{FLS}}=d \cdot d^{2} \sum_{m \mid d} m,
$$

where $\Delta$ denotes the class of the diagonal in $A \times A$. The divisor $\delta_{1}$ is associated with the locus of curves which split into genus 1 and genus 2 components. Since, generically, $A$ contains no genus 1 curves, the class on the genus 1 component must be 0 . Since $\left[\bar{M}_{1,1}(A, 0)\right]^{\mathrm{vir}}=0$, we obtain the third evaluation.

Proof of Proposition 1.4. Let $A$ and $\beta$ be generic. By Lemma 6.2, the only contribution to $\mathrm{H}_{g,(1, d)}^{\mathrm{FLS}}$ from maps $f: C \rightarrow A$ with $C$ nodal arises from the locus in $\bar{M}_{3}$ with a separating node. The maps are of the form

$$
f: B \cup C^{\prime} \rightarrow A
$$

where $B$ is a genus 2 curve and $C^{\prime}$ is an elliptic tail glued to $B$ along one of the six Weierstrass points of $B$. Under $f$, the curve $B$ maps to a genus 2 curve in $A$, while $C^{\prime}$ gets contracted. By a direct calculation (or examining the case $d=1$ ), we find that each genus 2 curve in the FLS contributes

$$
6 \cdot \frac{1}{2} \int_{\bar{M}_{1,1}} c_{1}(\mathrm{Ob})=-\frac{1}{4}
$$

where $\mathrm{Ob}$ denotes the obstruction sheaf. Therefore,

$$
\mathrm{h}_{3, \beta}^{A, \mathrm{FLS}}=\mathrm{H}_{3,(1, d)}^{\mathrm{FLS}}+d^{2} \sigma(d) \cdot \frac{1}{4}=d^{2} \sum_{m \mid d} \frac{m\left(3 m^{2}+1-4 d\right)}{4} .
$$

\subsection{A formula for all genera}

Consider the composition

$$
\operatorname{Hilb}^{2}(A) \rightarrow \operatorname{Sym}^{2}(A) \rightarrow A
$$

of the Hilbert-Chow morphism and the addition map. The fiber of $0_{A} \in A$ is the Kummer $K 3$ surface of $A$, denoted by $\operatorname{Km}(A)$. Alternatively, $\operatorname{Km}(A)$ can be defined as the blowup of $A / \pm 1$ at the 16 singular points.

In the notation of Section 6.2 , a map $\phi: \mathbb{P}^{1} \rightarrow \operatorname{Hilb}^{2}(A)$ not contained in $\Delta$ maps to $\operatorname{Km}(A)$ if and only if the corresponding hyperelliptic curve $f: C \rightarrow A$ maps a Weierstrass point of $C$ to a 2-torsion point of $A$.

By Nakajima's theorem on the cohomology of Hilbert schemes, we have a natural decomposition

$$
H_{2}\left(\operatorname{Hilb}^{2}(A) ; \mathbb{Z}\right)=H_{2}(A, \mathbb{Z}) \oplus \wedge^{2} H_{1}(A, \mathbb{Z}) \oplus \mathbb{Z} \cdot X,
$$

where $X$ is the class of an exceptional curve.

A hyperelliptic curve $f: C \rightarrow A$ in class $\beta$ corresponds to a map $\phi: \mathbb{P}^{1} \rightarrow \operatorname{Hilb}^{2}(A)$ not contained in $\Delta$, which has class

$$
\beta+\gamma+k X \in H_{2}\left(\operatorname{Hilb}^{2}(A), \mathbb{Z}\right)
$$




\section{CURVE COUNTING ON ABELIAN SURFACES AND THREEFOLDS}

for some $\gamma \in \wedge^{2} H_{1}(A, \mathbb{Z})$, with

$$
k=\chi\left(\mathcal{O}_{\bar{C}}\right)-2=-1-g_{a}(\bar{C}),
$$

where $g_{a}(\bar{C})$ is the arithmetic genus of $\bar{C}$; see [Obe18, Section 1.3].

Proposition 6.4. Let $\beta$ be an irreducible curve class of type $(1, d)$ on an abelian surface $A$ satisfying $(\dagger)$. Then, after the change of variables $y=-e^{2 \pi i z}$ and $q=e^{2 \pi i \tau}$, we have

$$
\sum_{g \geqslant 2} \mathrm{~h}_{g, \beta}^{A, \mathrm{FLS}}\left(y^{1 / 2}+y^{-1 / 2}\right)^{2 g+2}=\frac{d^{2}}{16} \text { Coeff }_{q^{d}}\left[4 K(z, \tau)^{4}\right],
$$

where Coeff $q^{d}$ denotes the coefficient of $q^{d}$.

Proof. For every hyperelliptic curve $f: C \rightarrow A$ in class $\beta$, the map $(p, f): C \rightarrow \bar{C}$ is an isomorphism by (†). In particular, the arithmetic genus of $\bar{C}$ is equal to the genus of $C$.

Hence, there is a bijective correspondence between

(i) maps $f: C \rightarrow A$ from non-singular hyperelliptic curves of genus $g$ and class $\beta$ for which a Weierstrass point of $C$ is mapped to a 2-torsion point of $A$,

(ii) maps $\phi: \mathbb{P}^{1} \rightarrow \operatorname{Hilb}^{2}(A)$ with image not contained in $\Delta$ of class

$$
\beta+\gamma-(g+1) X
$$

for some $\gamma \in \wedge^{2} H_{1}(A, \mathbb{Z})$ and with image in $\operatorname{Km}(A)$.

Let $\mathrm{h}_{g, \beta}^{A, H i l b}$ be the finite number of such curves.

In every translation class of a hyperelliptic curve $f: C \rightarrow A$ in class $\beta$, there are $d^{2}$ members (up to automorphisms) in a given fixed linear system, and 16 members (up to automorphisms) with a Weierstrass point of $C$ mapping to a 2-torsion point. Hence, we have

$$
\mathrm{h}_{g, \beta}^{A, \mathrm{FLS}}=\frac{d^{2}}{16} \mathrm{~h}_{g, \beta}^{A, \mathrm{Hilb}} .
$$

By assumption $(\dagger)$ and Lemma 6.1 , every map $\phi: \mathbb{P}^{1} \rightarrow \operatorname{Hilb}^{2}(A)$ as in item (ii) meets $\Delta$ transversely and is isolated. In this situation, Graber in [Gra01, Sections 2 and 3] has explicitly determined the relationship between the genus 0 Gromov-Witten invariants of $\operatorname{Hilb}^{2}(A)$ and the number of these rational curves.

Let $p: \bar{M}_{0}\left(\operatorname{Hilb}^{2}(A)\right) \rightarrow A$ be the map induced by (6.1). Then,

$$
\sum_{g \geqslant 0} \mathrm{~h}_{g, \beta}^{A, \mathrm{Hilb}}\left(y^{1 / 2}+y^{-1 / 2}\right)^{2 g+2}=\sum_{k \in \mathbb{Z}} \sum_{\gamma \in \wedge^{2} H_{1}(A, \mathbb{Z})} y^{k} \int_{\left[\bar{M}_{0}\left(\operatorname{Hilb}^{2}(A), \beta+\gamma+k A\right)\right]^{\mathrm{red}}} p^{*}\left(0_{A}\right) .
$$

The integral on the right-hand side reduces to the genus 0 invariants of the Kummer $K 3$ surfaces and is determined by the Yau-Zaslow formula. Direct calculations and theta function identities, see [Obe18] for details, then provide the closed evaluation

$$
\sum_{\substack{k \in \mathbb{Z} \\ \gamma \in \wedge^{2} H_{1}(A, \mathbb{Z})}} y^{k} \int_{\left[\bar{M}_{0}\left(\operatorname{Hilb}^{2}(A), \beta+\gamma+k A\right)\right]^{\mathrm{red}}} p^{*}\left(0_{A}\right)=\operatorname{Coeff}_{q^{d}}\left[4 K(z, \tau)^{4}\right] .
$$

We are now ready to prove Theorem 1.5.

Proof of Theorem 1.5. Let $\beta$ be an irreducible class of type $(1, d)$ on an abelian surface $A$ satisfying $(\dagger)$. The only contributions to $\mathrm{H}_{g,(1, d)}^{\mathrm{FLS}}$ from maps $f: C \rightarrow A$ with $C$ nodal are of the form 


$$
f: B \cup C_{1} \cup \cdots \cup C_{2 h+2} \rightarrow A
$$

where

- $f: B \rightarrow A$ is a map in class $\beta$ from a non-singular hyperelliptic curve $B$ of some genus $h<g$;

- the $C_{i}$ are pairwise disjoint curves that are glued to the $i$ th Weierstrass point $x_{i}$ of $B$ and are contracted under $f$;

- the genera $g_{i}$ of $C_{i}$ satisfy $h+g_{1}+\cdots+g_{2 h+2}=g$;

- if $g_{i} \geqslant 2$, then $C_{i}$ is a hyperelliptic curve and $x_{i}$ is a Weierstrass point of $C_{i}$.

By stability, the case $g_{i}=0$ does not appear.

For $g \geqslant 2$, let $\mathcal{H}_{g, 1} \in A^{g-1}\left(\bar{M}_{g, 1}\right)$ be the stack fundamental class of the closure of nonsingular hyperelliptic curves with marked point at a Weierstrass point. By convention, we set $\mathcal{H}_{1,1}=\frac{1}{2}\left[M_{1,1}\right]$. Then, the contribution of a non-singular hyperelliptic curve $f: B \rightarrow A$ of genus $h$ in class $\beta$ to $\mathrm{H}_{g,(1, d)}^{\mathrm{FLS}}$ is

$$
\sum_{\substack{g_{1}, \ldots, g_{2 h+2} \geqslant 0 \\ g_{1}+\cdots+g_{2 h+2}=g-h}} \prod_{\substack{i=1 \\ g_{i}>0}}^{2 h+2} \int_{\bar{M}_{g_{i}, 1}} \mathcal{H}_{g_{i}, 1} \cup c(\mathrm{Ob}),
$$

where $\mathrm{Ob}$ denotes the obstruction sheaf. Analyzing the tangent obstruction sequence, we obtain

$$
c(\mathrm{Ob})=\frac{c\left(\mathbb{E}^{\vee}\right)^{2}}{1-\psi_{1}}
$$

Define the generating series

$$
F(u)=u+\sum_{g \geqslant 1} u^{2 g+1} \int_{\bar{M}_{g, 1}} \frac{\mathcal{H}_{g, 1} \cup c\left(\mathbb{E}^{\vee}\right)^{2}}{1-\psi_{1}} .
$$

Then, from relation (6.3) and the definition of $\mathrm{h}_{g, \beta}^{A, \mathrm{FLS}}$, we obtain

$$
\sum_{h \geqslant 2} \mathrm{~h}_{h, \beta}^{A, \mathrm{FLS}} F(u)^{2 h+2}=\sum_{g \geqslant 2} \mathrm{H}_{g,(1, d)}^{\mathrm{FLS}} u^{2 g+2} .
$$

The series $F(u)$ has been computed by Wise using orbifold Gromov-Witten theory [Wis16]. The result is

$$
F(u)=2 \sin (u / 2)=u-\frac{1}{24} u^{3}+\frac{1}{1920} u^{5} \pm \cdots .
$$

Together with Proposition 6.4, this implies the claim.

The calculation of the invariants $\mathrm{H}_{g,(1, d)}^{\mathrm{FLS}}$ is similar to the calculations of the orbifold genus 0 Gromov-Witten theory of the second symmetric product of a non-singular surface as pursued in [Ros14, Wis11]. We expect that a connection can be made to their work.

The main step in the proof of Theorem 1.5 is the evaluation of the generating series $F(u)$. Below, we will give a second proof of Theorem 1.5 under slightly stronger assumptions. The main new input here is the refined modularity statement of Theorem 5.1. Using the modularity property, the evaluation of $F(u)$ will follow automatically from the theory of modular forms.

For the second proof, we will assume that the following holds: 


\section{CuRve COUNTING ON ABELIAN SURFACES AND THREEFOLDS}

$(\exists \dagger)$ For every $d \geqslant 1$, there exist an abelian surface $A$ and an irreducible curve class $\beta$ of type $(1, d)$ satisfying property $(\dagger)$.

Second proof of Theorem 1.5 under assumption $(\exists \dagger)$. For all $d \geqslant 1$, let $\beta_{d}$ be an irreducible class of type $(1, d)$ on an abelian surface $A_{d}$ satisfying $(\dagger)$.

Step 1. Define the generating series

$$
\varphi_{g}(q)=\sum_{d \geqslant 1} \mathrm{~h}_{g, \beta_{d}}^{A_{d}, \mathrm{FLS}} q^{d}
$$

By Proposition 6.4, we have, after the change of variables $u=2 \pi z$ and $y=-e^{i u}$,

$$
\sum_{g \geqslant 2}\left(y^{1 / 2}+y^{-1 / 2}\right)^{2 g+2} \varphi_{g}(q)=\left(q \frac{d}{d q}\right)^{2} \frac{K(z, \tau)^{4}}{4}=\sum_{m \geqslant 2} u^{2 m} f_{m}(q),
$$

where $f_{m}(q)$ are quasi-modular forms of weight $2 m$, that is, $f_{m}(q) \in \operatorname{QMod}_{2 m}$. Let $r=-\left(y^{1 / 2}+\right.$ $\left.y^{-1 / 2}\right)=2 \sin (u / 2)$, and let

$$
u=2 \arcsin (r / 2)=r+\frac{1}{24} r^{3}+\frac{3}{640} r^{5}+\cdots
$$

be the inverse transform. After inserting this into (6.4), we obtain

$$
\sum_{g \geqslant 2} \varphi_{g}(q) r^{2 g+2}=\sum_{m \geqslant 2}\left(r+\frac{1}{24} r^{3}+\cdots\right)^{2 m} f_{m}(q) .
$$

Hence, $\varphi_{g}(q)$ is a quasi-modular form with highest weight term $f_{g+1}(q)$ :

$$
\varphi_{g}(q)=f_{g+1}(q)+R(q)
$$

for $R(q) \in \mathrm{QMod}_{\leqslant 2 g}$.

Step 2. By the trade of the FLS for insertions as in (4.1), the vanishing of the $d=0$ term, and deformation invariance, we have

$$
\mathrm{F}_{g}^{E_{1} \times E_{2}}\left(\mathcal{H}_{g} ; \mathrm{a}_{1} \omega_{2}, \mathrm{~b}_{1} \omega_{2}, \omega_{1} \mathrm{a}_{2}, \omega_{1} \mathrm{~b}_{2}\right)=\sum_{d \geqslant 1} \mathrm{H}_{g,(1, d)}^{\mathrm{FLS}} q^{d}
$$

where we use the notation of Section 5. Applying Theorem 5.1, we see that the series (6.6) is hence a quasi-modular form of pure weight $2 g+2$.

Step 3. By assumption $(\dagger)$ and the discussion after (6.2), the Gromov-Witten invariant $\mathrm{H}_{g,(1, d)}^{\mathrm{FLS}}$ equals the sum

$$
\mathrm{H}_{g,(1, d)}^{\mathrm{FLS}}=\sum_{2 \leqslant g^{\prime} \leqslant g} c_{g^{\prime}, g} \mathrm{~h}_{g^{\prime}, \beta_{d}}^{A_{d}, \mathrm{FLS}}
$$

for coefficients $c_{g^{\prime}, g} \in \mathbb{Q}$. Summing up (6.7) over all $d$, we obtain

$$
\sum_{d \geqslant 1} \mathrm{H}_{g,(1, d)}^{\mathrm{FLS}} q^{d}=\sum_{2 \leqslant g^{\prime} \leqslant g} c_{g^{\prime}, g} \varphi_{g^{\prime}}(q)
$$




\section{J. Bryan, G. Oberdieck, R. Pandharipande and Q. Yin}

The left-hand side is homogeneous of weight $2 g+2$, hence must equal the weight $2 g+2$ part of the right-hand side. Therefore, by (6.5), we have

$$
\sum_{d \geqslant 1} \mathrm{H}_{g,(1, d)}^{\mathrm{FLS}} q^{d}=f_{g+1}(q) .
$$

By the definition of the $f_{g+1}(q)$, this shows part (ii) of the theorem:

$$
\sum_{g \geqslant 2} u^{2 g+2} \sum_{d \geqslant 1} \mathrm{H}_{g,(1, d)}^{\mathrm{FLS}} q^{d}=\sum_{g \geqslant 2} u^{2 g} f_{g}(q)=\left(q \frac{d}{d q}\right)^{2} \frac{1}{4} K(z, \tau)^{4} .
$$

Comparing (6.8) with (6.4), we see that part (i) also follows.

\section{Part II. Abelian threefolds}

\section{Donaldson-Thomas theory}

\subsection{Overview}

Let $A$ be a generic abelian surface carrying a curve class $\beta_{d^{\prime}}$ of type $\left(1, d^{\prime}\right)$ with $d^{\prime}>0$, and let $E$ be a generic elliptic curve. Throughout Section 7, we will work with the abelian threefold $X=A \times E$.

Here, we compute the topological Euler characteristic of the stack $\operatorname{Hilb}^{n}\left(X,\left(\beta_{d^{\prime}}, d\right)\right) / X$ in the cases $d^{\prime} \in\{1,2\}$, proving Theorem 1.6. Next, we present a conjectural relationship between the Behrend function weighted Euler characteristic and the topological Euler characteristic via a simple sign change, and show how it implies Corollary* 1.7 . We discuss the motivation and plausibility for the conjecture.

Our computation here is parallel to the computation of the reduced Donaldson-Thomas invariants for $K 3 \times E$ in [Bry15]. We will frequently refer to results of [Bry15]. The technique used was developed by Bryan and Kool in [BK16].

\subsection{Notation}

Since the translation action of $X$ on $\operatorname{Hilb}^{n}\left(X,\left(\beta_{d^{\prime}}, d\right)\right)$ has finite stabilizer, the reduced Donaldson-Thomas invariants

$$
\mathrm{DT}_{n,\left(\beta_{d^{\prime}}, d\right)}=e\left(\operatorname{Hilb}^{n}\left(X,\left(\beta_{d^{\prime}}, d\right)\right) / X, \nu\right)=\sum_{k \in \mathbb{Z}} k \cdot e\left(\nu^{-1}(k)\right)
$$

and the topological (unweighted) Euler characteristics

$$
\widehat{\mathrm{DT}}_{n,\left(\beta_{d^{\prime}}, d\right)}=e\left(\operatorname{Hilb}^{n}\left(X,\left(\beta_{d^{\prime}}, d\right)\right) / X\right)
$$

are well defined. We have dropped the superscript $X$ in the notation for the Donaldson-Thomas invariants of Section 1.3.1.

We also use the shorthand notation

$$
\operatorname{Hilb}^{n, d^{\prime}, d}(X)=\operatorname{Hilb}^{n}\left(X,\left(\beta_{d^{\prime}}, d\right)\right)
$$

and the following bullet convention. 


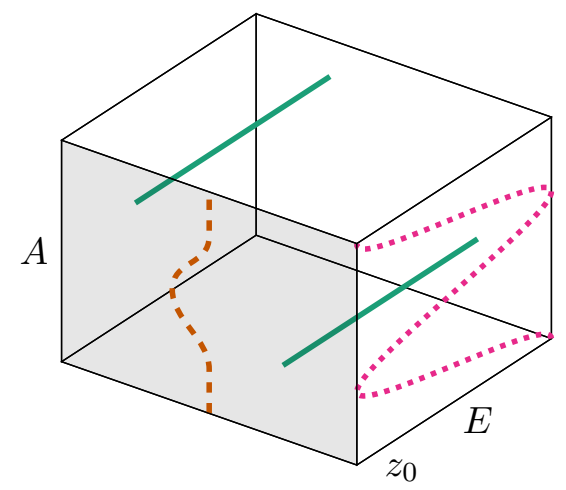

Figure 1. A vertical curve (dashed) contained in the slice $A \times\left\{z_{0}\right\}$ (light gray), a diagonal curve (dotted), and two horizontal curves (solid).

Convention. When an index in a space is replaced by a bullet $(\bullet)$, we sum over the index, multiplying by the appropriate variable. The result is a formal series with coefficients in the Grothendieck ring.

For example, we let

$$
\operatorname{Hilb}^{\bullet}, d^{\prime} \bullet \bullet(X) / X=\sum_{d \geqslant 0} \sum_{n \in \mathbb{Z}}\left[\operatorname{Hilb}^{n, d^{\prime}, d}(X) / X\right] p^{n} q^{d},
$$

which we regard as an element in $K_{0}\left(\mathrm{DM}_{\mathbb{C}}\right)((p))[[q]]$, the ring of formal power series in $q$, Laurent in $p$, with coefficients in the Grothendieck ring of Deligne-Mumford stacks over $\mathbb{C}$.

Define the Donaldson-Thomas partition functions of $X$,

$$
\begin{aligned}
& \mathrm{DT}_{d^{\prime}}=\sum_{d \geqslant 0} \sum_{n \in \mathbb{Z}} \mathrm{DT}_{n,\left(\beta_{d^{\prime}}, d\right)}(-p)^{n} q^{d}, \\
& \widehat{\mathrm{DT}}_{d^{\prime}}=\sum_{d \geqslant 0} \sum_{n \in \mathbb{Z}} \widehat{\mathrm{DT}}_{n,\left(\beta_{d^{\prime}}, d\right)} p^{n} q^{d} .
\end{aligned}
$$

By the bullet convention,

$$
\widehat{\mathrm{DT}}_{d^{\prime}}=e\left(\operatorname{Hilb}^{\bullet}, d^{\prime}, \bullet(X) / X\right),
$$

where we extend the Euler characteristic $e: K_{0}\left(\mathrm{DM}_{\mathbb{C}}\right) \rightarrow \mathbb{Q}$ termwise to the ring of formal power series in $p$ and $q$ over $K_{0}\left(\mathrm{DM}_{\mathbb{C}}\right)$.

\subsection{Vertical and diagonal loci}

Let $p_{A}$ and $p_{E}$ be the projections of $X=A \times E$ onto the factors $A$ and $E$, respectively. We say an irreducible curve $C \subset X$ is

- vertical if $p_{E}: C \rightarrow E$ has degree 0 ,

- horizontal if $p_{A}: C \rightarrow A$ has degree 0 ,

- diagonal if $p_{A}, p_{E}$ both have non-zero degree.

The various definitions are illustrated in Figure 1.

Consider a subscheme $C \subset X$ which defines a point in $\operatorname{Hilb}^{n, d^{\prime}, d}(X)$. Since the class $p_{A *}[C]=\beta_{d^{\prime}}$ is irreducible, there is a unique irreducible component of $C$ of dimension 1 , which is either vertical or diagonal. All other irreducible components of $C$ of dimension 1 are horizontal. 


\section{J. Bryan, G. Oberdieck, R. Pandharipande and Q. Yin}

Consider the sublocus

$$
\operatorname{Hilb}_{\text {vert }}^{n, d^{\prime}, d}(X) \subset \operatorname{Hilb}^{n, d^{\prime}, d}(X)
$$

parameterizing subschemes $C \subset X$ with $C_{0} \times\left\{z_{0}\right\} \subset C$ for some $z_{0} \in E$ and for some curve $C_{0} \subset A$ of class $\beta_{d^{\prime}}$. We endow $\operatorname{Hilb}_{\text {vert }}^{n, d^{\prime}, d}(X)$ with the natural scheme structure. It is a closed subscheme of $\operatorname{Hilb}^{n, d^{\prime}, d}(X)$.

Let $\operatorname{Hilb}_{\mathrm{diag}}^{n, d^{\prime}, d}(X)$ be the complement of the inclusion (7.1),

$$
\operatorname{Hilb}_{\text {diag }}^{n, d^{\prime}, d}(X)=\operatorname{Hilb}^{n, d^{\prime}, d}(X) \backslash \operatorname{Hilb}_{\text {vert }}^{n, d^{\prime}, d}(X) .
$$

Hence, every point in $\operatorname{Hilb}_{\mathrm{diag}}^{n, d^{\prime}, d}(X)$ corresponds to a subscheme $C \subset X$, which contains a diagonal component.

Since the condition defining the subscheme (7.1) is invariant under the translation action of $X$, we have an induced action of $X$ on $\operatorname{Hilb}_{\text {vert }}^{n, d^{\prime}, d}(X)$ and its complement. We exhibit the stack $\operatorname{Hilb}_{\text {vert }}^{n, d^{\prime}, d}(X) / X$ as a global quotient stack of a scheme by a finite group of order $d^{\prime 2}$, as follows.

Let $L \rightarrow A$ be a fixed line bundle on $A$ with $c_{1}(L)=\beta_{d^{\prime}}$, and let $z_{0} \in E$ be a fixed point. Consider the subscheme

$$
\operatorname{Hilb}_{\text {vert,fixed }}^{n, d^{\prime}, d}(X) \subset \operatorname{Hilb}_{\text {vert }}^{n, d^{\prime}, d}(X)
$$

parameterizing subschemes $C \subset X$ with $C_{0} \times\left\{z_{0}\right\} \subset C$ for some

$$
C_{0} \in|L| \text {. }
$$

The stabilizer of $\operatorname{Hilb}_{\mathrm{vert}, \text { fixed }}^{n, d^{\prime}, d}(X)$ under the translation action of $X$ is the subgroup

$$
\operatorname{Ker}(\phi: A \rightarrow \widehat{A}) \subset A,
$$

where $\phi: a \mapsto L \otimes t_{a}^{*} L^{-1}$ and $t_{a}: A \rightarrow A$ denotes the translation by $a \in A$. By (2.1), the subgroup (7.3) is isomorphic to $\mathbb{Z}_{d^{\prime}} \times \mathbb{Z}_{d^{\prime}}$. Hence, we have the stack equivalence

$$
\operatorname{Hilb}_{\text {vert }}^{n, d^{\prime}, d}(X) / X \cong \operatorname{Hilb}_{\text {vert }, \text { fixed }}^{n, d^{\prime}, d}(X) /\left(\mathbb{Z}_{d^{\prime}} \times \mathbb{Z}_{d^{\prime}}\right) \text {. }
$$

\subsection{Proof of Theorem 1.6(i)}

Let $L$ be a line bundle on $A$ with $c_{1}(L)=\beta_{1}$, and let $C_{0} \in|L|$ be the unique non-singular genus 2 curve in $|L|$. Since $L$ has type $(1,1)$, the class $c_{1}(L)$ is a principally polarization of $A$. In particular, $A$ is isomorphic to the Jacobian $J$ of $C_{0}$.

Step 1. Every irreducible diagonal curve $C \subset X$ in class $\left(\beta_{1}, d\right)$ maps isomorphically to $C_{0}$ and, therefore, induces a non-constant map $C_{0} \rightarrow E$. Dualizing, we obtain a non-constant map $E \rightarrow J\left(C_{0}\right) \cong A$, whose image is an abelian subvariety of $A$ of dimension 1 . Hence, by the genericity of $A$, no diagonal curve exists and $\operatorname{Hilb}_{\mathrm{diag}}^{n, d^{\prime}, d}(X)$ is empty.

Since there are no diagonal curves, we write

$$
\operatorname{Hilb}_{\text {fixed }}^{n, d^{\prime}, d}(X)=\operatorname{Hilb}_{\text {vert,fixed }}^{n, d^{\prime}, d}(X) .
$$

Then, by the equivalence (7.4) with $d^{\prime}=1$, we have

$$
e\left(\operatorname{Hilb}^{n, d^{\prime}, d}(X) / X\right)=e\left(\operatorname{Hilb}_{\text {fixed }}^{n, d^{\prime}, d}(X)\right) .
$$

Using the bullet convention, we find

$$
\widehat{\mathrm{DT}}_{1}=e\left(\operatorname{Hilb}_{\text {fixed }}^{\bullet, 1, \bullet}(X)\right) .
$$




\section{Curve COUnting on ABElian SURFACES AND THREefoldS}

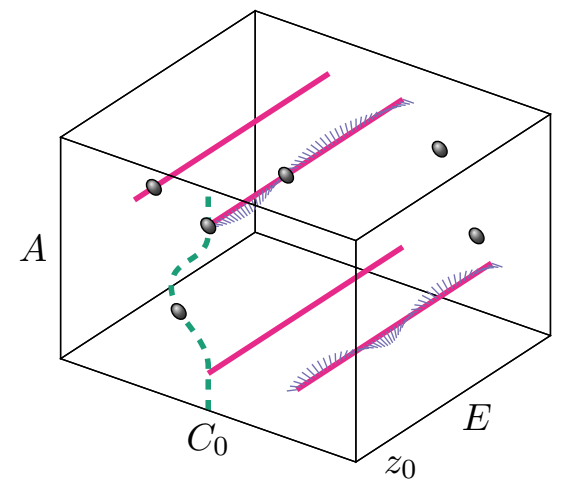

Figure 2. Subschemes in $A \times E$ up to translation. Horizontal curves (solid) can have nilpotent thickenings, and there can be embedded and floating points (gray). The unique vertical curve $C_{0} \in|L|$ (dashed) lies in $A \times\left\{z_{0}\right\}$ and is generically reduced.

Step 2. Let $\widehat{X}_{C_{0} \times E}$ be the formal completion of $X$ along the closed subvariety $C_{0} \times E$, and let $U=X \backslash C_{0} \times E$ be the open complement. The subschemes $\left\{\widehat{X}_{C_{0} \times E}, U\right\}$ forms a fpqc cover of $X$. By fpqc descent, subschemes in $X$ are determined by their restrictions to $\widehat{X}_{C_{0} \times E}$ and $U$. Since subschemes parameterized by $\operatorname{Hilb}_{\text {fixed }}^{n, 1, d}(X)$ are disjoint unions of components contained entirely in $\widehat{X}_{C_{0} \times E}$ or $U$, see Figure 2, there is no overlap condition for descent.

Consequently, we can stratify $\operatorname{Hilb}_{\text {fixed }}^{n, 1, d}(X)$ by locally closed subsets isomorphic to the product of Hilbert schemes of $\widehat{X}_{C_{0} \times E}$ and $U$, respectively. The result is succinctly expressed as an equality in the Grothendieck ring $K_{0}\left(\operatorname{Var}_{\mathbb{C}}\right)((p))[[q]]$ :

$$
\operatorname{Hilb}_{\text {fixed }}^{\bullet, 1, \bullet}(X)=\operatorname{Hilb}_{\text {fixed }}^{\bullet, 1, \bullet}\left(\widehat{X}_{C_{0} \times E}\right) \cdot \operatorname{Hilb}^{\bullet, 0, \bullet}(U),
$$

where we view $\operatorname{Hilb}_{\text {fixed }}^{n, 1, d}\left(\widehat{X}_{C_{0} \times E}\right)$ and $\operatorname{Hilb}^{n, 0, d}(U)$ as subschemes of $\operatorname{Hilb}_{\text {fixed }}^{n, 1, d}(X)$ and $\operatorname{Hilb}^{n, 0, d}(X)$, respectively. Taking Euler characteristics in (7.5), we obtain

$$
\widehat{\mathrm{DT}}_{1}=e\left(\operatorname{Hilb}_{\mathrm{fixed}}^{\bullet, 1, \bullet}\left(\widehat{X}_{C_{0} \times E}\right)\right) \cdot e\left(\operatorname{Hilb}^{\bullet, 0, \bullet}(U)\right) \text {. }
$$

Step 3. We calculate the second factor $e\left(\operatorname{Hilb}^{\bullet, 0, \bullet}(U)\right)$. The $E$-action on $U$ induces an action of $E$ on $\operatorname{Hilb}^{n, 0, d}(U)$. This new $E$-action exists because the fixed condition (7.2) concerns only the $\operatorname{Hilb}_{\text {fixed }}^{n, 1, d}\left(\widehat{X}_{C_{0} \times E}\right)$-factors and is independent of $U$ and $\operatorname{Hilb}^{n, 0, d}(U)$.

Since a scheme with a free $E$-action has trivial Euler characteristic, we have

$$
e\left(\operatorname{Hilb}^{n, 0, d}(U)\right)=e\left(\operatorname{Hilb}^{n, 0, d}(U)^{E}\right),
$$

where $\operatorname{Hilb}^{n, 0, d}(U)^{E}$ is the fixed locus of the $E$-action on $\operatorname{Hilb}^{n, 0, d}(U)$. Every element of $\mathrm{Hilb}^{n, 0, d}(U)^{E}$ corresponds to an $E$-invariant subscheme or, equivalently, is of the form $Z \times E$ for a 0-dimensional subscheme $Z \subset A \backslash C_{0}$ of length $d$. Since $\chi\left(\mathcal{O}_{Z \times E}\right)=0$ for every such $Z$, we find

$$
\begin{aligned}
e\left(\operatorname{Hilb}^{\bullet, 0, \bullet}(U)\right) & =e\left(\sum_{d \geqslant 0} \operatorname{Hilb}^{d}\left(A \backslash C_{0}\right) q^{d}\right) \\
& =\left(\prod_{m \geqslant 1}\left(1-q^{m}\right)^{-1}\right)^{e\left(A \backslash C_{0}\right)}=\prod_{m \geqslant 1}\left(1-q^{m}\right)^{-2} .
\end{aligned}
$$

We have used Göttsche's formula for the Euler characteristic of the Hilbert scheme of points of 
J. Bryan, G. Oberdieck, R. Pandharipande And Q. Yin

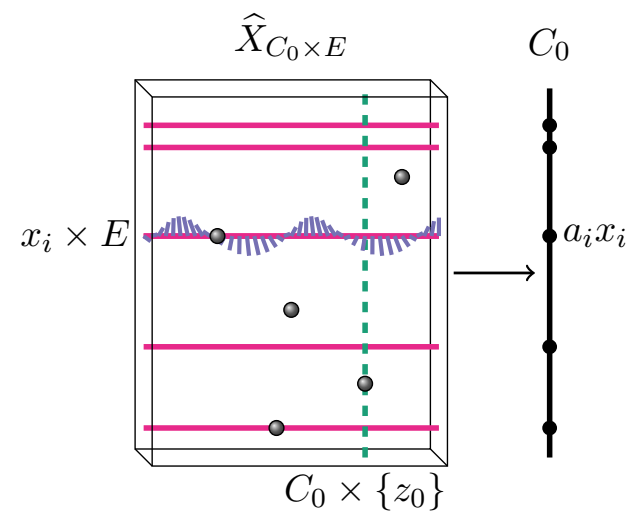

Figure 3. The map $\rho_{d}: \operatorname{Hilb}_{\text {fixed }}^{n, 1, d}\left(\widehat{X}_{C_{0} \times E}\right) \rightarrow \operatorname{Sym}^{d}\left(C_{0}\right)$ records the location and multiplicity of the horizontal curve components.

a surface [Göt90].

Step 4. We calculate the first factor $e\left(\operatorname{Hilb}_{\text {fixed }}^{\bullet, 1, \bullet}\left(\widehat{X}_{C_{0} \times E}\right)\right)$.

Consider the constructible morphism ${ }^{8}$

$$
\rho_{d}: \operatorname{Hilb}_{\text {fixed }}^{n, 1, d}\left(\widehat{X}_{C_{0} \times E}\right) \rightarrow \operatorname{Sym}^{d}\left(C_{0}\right),
$$

defined as follows. Let $[C] \in \operatorname{Hilb}_{\text {fixed }}^{n, 1, d}\left(\widehat{X}_{C_{0} \times E}\right)$ be a scheme with curve support $C_{0} \times z_{0} \cup_{i}\left(x_{i} \times E\right)$ and multiplicity $a_{i}$ along $x_{i} \times E$. Then

$$
\rho_{d}([C])=\sum_{i} a_{i} x_{i} \in \operatorname{Sym}^{d}\left(C_{0}\right) .
$$

Hence, $\rho_{d}([C])$ records the intersection (with multiplicities) of $C_{0}$ with all horizontal components of $C$; see Figure 3.

We determine the Euler characteristic of $\operatorname{Hilb}_{\text {fixed }}^{\bullet, 1, \bullet}\left(\widehat{X}_{C_{0} \times E}\right)$ by computing the Euler characteristic of $\operatorname{Sym}^{d}\left(C_{0}\right)$, weighted by the constructible function given by the Euler characteristic of the fibers of $\rho_{d}$. Hence, we write

$$
e\left(\operatorname{Hilb}_{\text {fixed }}^{n, 1, d}\left(\widehat{X}_{C_{0} \times E}\right)\right)=\int_{\operatorname{Hilb}_{\text {fixed }}^{n, 1, d}\left(\widehat{X}_{C_{0} \times E}\right)} 1 d e=\int_{\operatorname{Sym}^{d} C_{0}}\left(\rho_{d}\right)_{*}(1) d e,
$$

where $d e$ is the measure on constructible subsets induced by the Euler characteristic and $\rho_{d *}(1)$ denotes integration along the fiber. By writing

$$
\operatorname{Sym}^{\bullet} C_{0}=\sum_{d \geqslant 0} \operatorname{Sym}^{d} C_{0} q^{d}
$$

and extending the integration to the bullet notation termwise, we obtain

$$
e\left(\operatorname{Hilb}_{\text {fixed }}^{\bullet, 1, \bullet}\left(\widehat{X}_{C_{0} \times E}\right)\right)=\int_{\mathrm{Sym}_{C_{0}}} \rho_{*}(1) d e,
$$

\footnotetext{
${ }^{8} \mathrm{~A}$ constructible morphism is a map which is regular on each piece of a decomposition of its domain into locally closed subsets. Because we work with Euler characteristics and the Grothendieck group, we only need to work with constructible morphisms.
} 
where the measureable function $\rho_{*}(1)$ is given by

$$
\rho_{*}(1)\left(\sum_{i} a_{i} x_{i}\right)=e\left(\rho^{-1}\left(\sum_{i} a_{i} x_{i}\right)\right) \in \mathbb{Z}((p)) .
$$

The following result shows that $\rho_{*}(1)$ depends only on the underlying partition of the point in the symmetric product.

Proposition 7.1. We have

$$
\rho_{*}(1)\left(\sum_{i} a_{i} x_{i}\right)=\left(p^{1 / 2}(1-p)^{-1}\right)^{e\left(C_{0}\right)} \prod_{i} F\left(a_{i}\right),
$$

where

$$
\sum_{a \geqslant 0} F(a) q^{a}=\prod_{m \geqslant 1} \frac{\left(1-q^{m}\right)}{\left(1-p q^{m}\right)\left(1-p^{-1} q^{m}\right)} .
$$

The proof of Proposition 7.1 is identical to the proof of [Bry15, Proposition 4.1 and Lemma 4.3] with $e\left(C_{0}\right)=-2$ here (instead of Euler characteristic 2 in [Bry15]).

We apply the following result regarding weighted Euler characteristics of symmetric products.

Lemma 7.2. Let $S$ be a scheme, and let $\operatorname{Sym}^{\bullet}(S)=\sum_{d \geqslant 0} \operatorname{Sym}^{d}(S) q^{d}$. Let $G$ be a constructible function on $\operatorname{Sym}^{\bullet}(S)$ such that

$$
G\left(\sum_{i} a_{i} x_{i}\right)=\prod_{i} g\left(a_{i}\right)
$$

for a function $g$ with $g(0)=1$. Then

$$
\int_{\mathrm{Sym}} \cdot S d e=\left(\sum_{a \geqslant 0} g(a) q^{a}\right)^{e(S)} .
$$

An elementary proof of Lemma 7.2 is given in [BK16], but see also [Bry15, Lemma 4.2].

After applying Proposition 7.1 and Lemma 7.2 to (7.9), we obtain

$$
\begin{aligned}
e\left(\operatorname{Hilb}_{\text {fixed }}^{\bullet, 1, \bullet}\left(\widehat{X}_{C_{0} \times E}\right)\right) & =p^{-1}(1-p)^{2}\left(\sum_{a \geqslant 0} F(a) q^{a}\right)^{-2} \\
& =p^{-1}(1-p)^{2} \prod_{m \geqslant 1} \frac{\left(1-p q^{m}\right)^{2}\left(1-p^{-1} q^{m}\right)^{2}}{\left(1-q^{m}\right)^{2}}
\end{aligned}
$$

Using (7.6), (7.7), and the definition of $K(p, q)$, we obtain the evaluation of part (i) of Theorem 1.6.

\subsection{Proof of Theorem 1.6(ii)}

Let $A$ be a generic abelian surface with curve class $\beta_{2}$ of type $(1,2)$, and let $L \rightarrow A$ be a fixed line bundle with $c_{1}(L)=\beta_{2}$. The linear system $|L|=\mathbb{P}^{1}$ is a pencil of irreducible genus 3 curves. The generic curve in the pencil is non-singular, but there are exactly 12 singular curves (each of which has a single nodal); see [BL99].

By the disjoint union

$$
\operatorname{Hilb}^{n, d^{\prime}, d}(X)=\operatorname{Hilb}_{\mathrm{diag}}^{n, d^{\prime}, d}(X) \sqcup \operatorname{Hilb}_{\text {vert }}^{n, d^{\prime}, d}(X)
$$




\section{J. Bryan, G. Oberdieck, R. Pandharipande and Q. Yin}

and the isomorphism (7.4), we have

$$
\operatorname{Hilb}^{n, 2, d}(X) / X=\operatorname{Hilb}_{\text {vert,fixed }}^{n, 2, d}(X) /(\mathbb{Z} / 2 \times \mathbb{Z} / 2) \sqcup \operatorname{Hilb}_{\text {diag }}^{n, 2, d}(X) / X .
$$

Using the bullet convention, it follows that we have

$$
\widehat{\mathrm{DT}}_{2}=\frac{1}{4} e\left(\operatorname{Hilb}_{\text {vert }, \text { fixed }}^{\bullet,, \bullet}(X)\right)+e\left(\operatorname{Hilb}_{\mathrm{diag}}^{\bullet, 2, \bullet}(X) / X\right) .
$$

Step 1. We begin by evaluating $e\left(\operatorname{Hilb}_{\text {vert,fixed }}^{\bullet, 2, \bullet}(X)\right)$. Consider the map

$$
\tau: \operatorname{Hilb}_{\text {vert,fixed }}^{\bullet, 2, \bullet}(X) \rightarrow|L|=\mathbb{P}^{1},
$$

which maps a subscheme $C$ to the divisor in $|L|$ associated with $p_{A}(C)$. The fiber of $\tau$ over a point $C \in|L|$, denoted by

$$
\operatorname{Hilb}_{C}^{n, 2, d}(X) \subset \operatorname{Hilb}_{\text {vert,fixed }}^{n, 2, d}(X),
$$

is the sublocus of $\operatorname{Hilb}_{\text {vert,fixed }}^{n, 2, d}(X)$ which parameterizes curves which contain the curve $C \times\left\{z_{0}\right\}$.

As we have done in (7.9), we may write

$$
e\left(\operatorname{Hilb}_{\text {vert,fixed }}^{\bullet, 2, \bullet}(X)\right)=\int_{|L|} \tau_{*}(1) d e,
$$

where $\tau_{*}(1)$ denotes the constructible function obtained by integration along the fiber:

$$
\tau_{*}(1)([C])=e\left(\operatorname{Hilb}_{C}^{\bullet, 2, \bullet}(X)\right) .
$$

Step 2. Let $C \subset A$ be a curve in $|L|$. Following a strategy similar to that of the proof of part (i), we will compute explicit expressions for $\tau_{*}(1)([C])$ depending only upon whether $C$ is nodal or not.

Following Step 2 of the proof of part (i), we have

$$
\operatorname{Hilb}_{C}^{\bullet, 2, \bullet}(X)=\operatorname{Hilb}_{C}^{\bullet, 2, \bullet}\left(\widehat{X}_{C \times E}\right) \cdot \operatorname{Hilb}_{C}^{\bullet, 2, \bullet}(X \backslash C \times E) .
$$

Using the extra $E$-action on the second factors, we obtain

$$
e\left(\operatorname{Hilb}_{C}^{\bullet, 2, \bullet}(X)\right)=e\left(\operatorname{Hilb}_{C}^{\bullet, 2, \bullet}\left(\widehat{X}_{C \times E}\right)\right) \cdot \prod_{m \geqslant 1}\left(1-q^{m}\right)^{-e(A \backslash C)} .
$$

For the first factor, we use the map

$$
\rho: \operatorname{Hilb}_{C}^{\bullet, 2, \bullet}\left(\widehat{X}_{C \times E}\right) \rightarrow \operatorname{Sym}^{\bullet}(C),
$$

which records the location and multiplicity of the horizontal components (and has already appeared in (7.8)). 


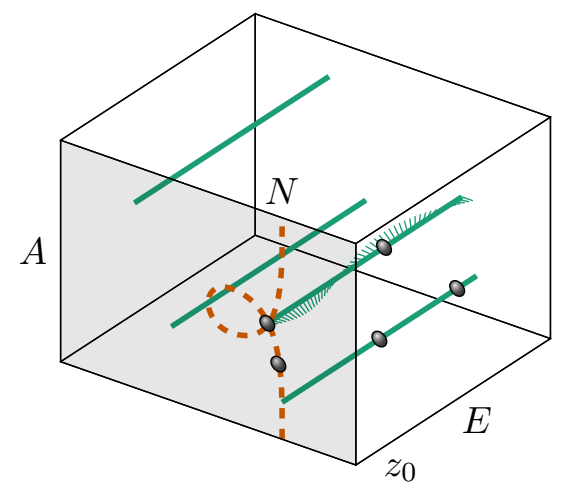

Figure 4. A subscheme parameterized by $\operatorname{Hilb}_{N}^{\bullet, 2, \bullet}(X)$ which includes a thickened horizontal curve (solid) attached to the node of a nodal vertical curve (dashed). For the subscheme to have a non-zero contribution to the Euler characteristic, embedded points (gray) can only occur on $N$ or on horizontal curves attached to $N$.

Step 3. If $C$ is non-singular, we apply Proposition 7.1 with $C$ in place of $C_{0}$ for the integration along the fiber of $\rho$. By Lemma 7.2, we have

$$
\begin{aligned}
e\left(\operatorname{Hilb}_{C}^{\bullet, 2, \bullet}\left(\widehat{X}_{C \times E}\right)\right) & =\int_{\operatorname{Sym}^{\bullet} C} \rho_{*}(1) d e \\
& =\left(p^{1 / 2}(1-p)^{-1}\right)^{e(C)}\left(\sum_{a \geqslant 0} F(a) q^{a}\right)^{e(C)} \\
& =p^{-2}(1-p)^{4} \prod_{m \geqslant 1} \frac{\left(1-p q^{m}\right)^{4}\left(1-p^{-1} q^{m}\right)^{4}}{\left(1-q^{m}\right)^{4}} .
\end{aligned}
$$

Using (7.11) with $e(A \backslash C)=4$, we find

$$
\tau_{*}(1)([C])=e\left(\operatorname{Hilb}_{C}^{\bullet, 2, \bullet}(X)\right)=K(p, q)^{4} .
$$

Step 4. Let $C=N \in|L|$ be a curve with a nodal point $z \in C$. The corresponding moduli space $\operatorname{Hilb}_{N}^{n, 2, d}(X)$ is depicted in Figure 4. We have the following result.

Proposition 7.3. Let $x_{1}, \ldots, x_{l} \in N \backslash\{z\}$; then

$$
\rho_{*}(1)\left(b z+\sum_{i=1}^{l} a_{i} x_{i}\right)=p^{-2}(1-p)^{4} N(b) \prod_{i=1}^{l} F\left(a_{i}\right),
$$

where

$$
\sum_{b \geqslant 0} N(b) q^{b}=\prod_{m \geqslant 1}\left(1-q^{m}\right)^{-1} \cdot\left(1+\frac{p}{(1-p)^{2}}+\sum_{d \geqslant 1} \sum_{k \mid d} k\left(p^{k}+p^{-k}\right) q^{d}\right) .
$$

The proof is identical to the proof of the corresponding statement for contributions of nodal curves in the $K 3 \times E$-geometry of [Bry15, Section 5]. The only difference is that in our case, $e(N \backslash\{z\})=-4$, whereas in the $K 3$ case, $e(N \backslash\{z\})=0$. The different Euler characteristic results in the different prefactor $p^{-2}(1-p)^{4}$ in (7.13). The prefactor in general is

$$
\left(p^{1 / 2}(1-p)^{-1}\right)^{e(N \backslash\{z\})} \text {. }
$$




\section{J. Bryan, G. Oberdieck, R. Pandharipande and Q. Yin}

The geometry of the term $N(b)$ arises as the contribution

$$
N(b)=e\left(\operatorname{Hilb}^{\bullet, 2, b}\left(\widehat{X}_{\{z\} \times E}\right)\right) .
$$

In [Bry15], the right-hand side is expressed in terms of the topological vertex. By results of [BO00], we obtain the closed form of Proposition 7.3.

By Proposition 7.3 and Lemma 7.2, we obtain

$$
\begin{aligned}
e\left(\operatorname{Hilb}_{N}^{\bullet, 2, \bullet}\left(\widehat{X}_{N \times E}\right)\right)= & \int_{\operatorname{Sym} \bullet N} \rho_{*}(1) d e \\
= & p^{-2}(1-p)^{4} \int_{\operatorname{Sym} \bullet(N \backslash\{z\})} \prod_{i} F\left(a_{i}\right) d e \int_{\operatorname{Sym} \bullet(\{z\})} N(b) d e \\
= & p^{-2}(1-p)^{4}\left(\sum_{a \geqslant 0} F(a) q^{a}\right)^{e(N \backslash\{z\})}\left(\sum_{b \geqslant 0} N(b) q^{b}\right) \\
= & p^{-2}(1-p)^{4}\left(\prod_{m \geqslant 1} \frac{\left(1-p q^{m}\right)^{4}\left(1-p^{-1} q^{m}\right)^{4}}{\left(1-q^{m}\right)^{5}}\right) \\
& \cdot\left(1+\frac{p}{(1-p)^{2}}+\sum_{d \geqslant 1} \sum_{k \mid d} k\left(p^{k}+p^{-k}\right) q^{d}\right) .
\end{aligned}
$$

By (7.11) with $e(A \backslash N)=3$, we find

$$
\begin{aligned}
\tau_{*}(1)([N]) & =e\left(\operatorname{Hilb}_{N}^{\bullet, 2, \bullet}(X)\right) \\
& =K(p, q)^{4} \cdot\left(1+\frac{p}{(1-p)^{2}}+\sum_{d \geqslant 1} \sum_{k \mid d} k\left(p^{k}+p^{-k}\right) q^{d}\right) .
\end{aligned}
$$

Step 5. We complete the calculation of $e\left(\operatorname{Hilb}_{\text {vert,fixed }}^{\bullet, 2, \bullet}(X)\right)$.

By (7.12) and (7.14), the function $\tau_{*}(1)([C])$ depends only upon whether $C \in|L|$ is nodal or not. Therefore,

$$
\begin{aligned}
e\left(\operatorname{Hilb}_{\text {vert,fixed }}^{\bullet, 2, \bullet}(X)\right) & =\int_{|L|} \tau_{*}(1) d e \\
& =e\left(\mathbb{P}^{1} \backslash 12 \text { points }\right) \cdot K(p, q)^{4}+e(12 \text { points }) \cdot \tau_{*}(1)(N) \\
& =-10 K(p, q)^{4}+12 K(p, q)^{4} \cdot\left(1+\frac{p}{(1-p)^{2}}+\sum_{d \geqslant 1} \sum_{k \mid d} k\left(p^{k}+p^{-k}\right) q^{d}\right) \\
& =K(p, q)^{4} \cdot\left(2+12 \frac{p}{(1-p)^{2}}+12 \sum_{d \geqslant 1} \sum_{k \mid d} k\left(p^{k}+p^{-k}\right) q^{d}\right) .
\end{aligned}
$$

Step 6. We compute the contribution $e\left(\operatorname{Hilb}_{\mathrm{diag}}^{\bullet, 2, \bullet}(X) / X\right)$ arising from the locus of curves with a diagonal component.

By Lemma 8.6, there are $12 \sigma(d / 2) \delta_{d \text {,even }}$ isolated translation classes of diagonal curves of class $\left(\beta_{2}, d\right)$. Moreover, the translation action of $X$ on each translation class is free.

Choose one representative from each $X$-orbit of the diagonal classes. Let

$$
\operatorname{Hilb}_{\text {diag,fixed }}^{n, 2, d}(X) \subset \operatorname{Hilb}_{\text {diag }}^{n, 2, d}(X)
$$

be the subscheme parameterizing curves which contain one of the chosen representatives. The 


\section{CURVE COUNTING ON ABELIAN SURFACES AND THREEFOLDS}

moduli space (7.15) defines a slice for the action of $X$ on $\operatorname{Hilb}_{\mathrm{diag}}^{n, 2, d}(X)$,

$$
\operatorname{Hilb}_{\mathrm{diag}}^{n, 2, d}(X) / X \cong \operatorname{Hilb}_{\mathrm{diag}, \text { fixed }}^{n, 2, d}(X) .
$$

The contribution of such subschemes to the Euler characteristic is computed precisely as the contribution with a genus 3 vertical component in Step 3 above. Taking into account the number of diagonal curves and their degrees in the horizontal direction, we find

$$
\begin{aligned}
e\left(\operatorname{Hilb}_{\text {diag,fixed }}^{\bullet, 2, \bullet}(X)\right) & =e\left(\operatorname{Hilb}_{C}^{\bullet, 2, \bullet}(X)\right) \cdot\left(12 \sum_{d \geqslant 1} \sum_{k \mid d} k q^{2 d}\right) \\
& =K(p, q)^{4} \cdot\left(12 \sum_{d \geqslant 1} \sum_{k \mid d} k q^{2 d}\right) .
\end{aligned}
$$

Step 7. We have calculated all terms in the sum (7.10) in Steps 5 and 6. After summing, the proof of part (ii) of Theorem 1.6 is complete.

\subsection{The Behrend function}

In the cases $d^{\prime} \in\{1,2\}$, we conjecture that the Behrend function weighted Euler characteristic of the Hilbert schemes differs from the ordinary Euler characteristic by a factor of $\pm(-1)^{n}$. Here, $n$ is the holomorphic Euler characteristic, and the overall sign depends upon whether the component of the Hilbert scheme corresponds to subschemes with diagonal curves or vertical curves.

The Behrend function on the quotient,

$$
\nu: \operatorname{Hilb}^{n, d^{\prime}, d}(X) / X \rightarrow \mathbb{Z},
$$

induces, by our identification of the various components with different slices of the $X$-action, constructible functions on

$$
\operatorname{Hilb}_{\text {fixed }}^{n, 1, d}(X), \quad \operatorname{Hilb}_{\text {vert,fixed }}^{n, 2, d}(X), \quad \text { and } \quad \operatorname{Hilb}_{\text {diag,fixed }}^{n, 2, d}(X) .
$$

We will denote these functions by $\nu$ as well and write $e(\cdot, \nu)$ for the topological Euler characteristic weighted by $\nu$.

Conjecture D. We have

$$
\begin{gathered}
e\left(\operatorname{Hilb}_{\text {fixed }}^{n, 1, d}(X)\right)=-(-1)^{n} e\left(\operatorname{Hilb}_{\text {fixed }}^{n, 1, d}(X), \nu\right), \\
e\left(\operatorname{Hilb}_{\text {vert }, \text { fixed }}^{n, 2,}(X)\right)=-(-1)^{n} e\left(\operatorname{Hilb}_{\text {vert,fixed }}^{n, 2, d}(X), \nu\right), \\
e\left(\operatorname{Hilb}_{\text {diag,fixed }}^{n, 2, d}(X)\right)=+(-1)^{n} e\left(\operatorname{Hilb}_{\text {diag,fixed }}^{n, 2, d}(X), \nu\right) .
\end{gathered}
$$

Assuming Conjecture D, we prove Corollary* 1.7 .

Proof of Corollary* 1.7. In case $d^{\prime}=1$, by Conjecture D, we have $\mathrm{DT}_{1}=-\widehat{\mathrm{DT}}_{1}$. Part (i) of Corollary* 1.7 hence follows from part (i) of Theorem 1.6.

In case $d^{\prime}=2$, we have, following (7.10),

$$
\mathrm{DT}_{2}=\sum_{d \geqslant 0} \sum_{n \in \mathbb{Z}}\left(\frac{1}{4} e\left(\operatorname{Hilb}_{\text {vert,fixed }}^{\bullet, 2, \bullet}(X), \nu\right)+e\left(\operatorname{Hilb}_{\text {diag,fixed }}^{\bullet, 2, \bullet}(X), \nu\right)\right)(-p)^{n} q^{d} .
$$

By Conjecture D, the right side equals

$$
\sum_{d \geqslant 0} \sum_{n \in \mathbb{Z}}\left(-\frac{1}{4} e\left(\operatorname{Hilb}_{\text {vert,fixed }}^{\bullet, 2, \bullet}(X)\right)+e\left(\operatorname{Hilb}_{\text {diag,fixed }}^{\bullet, 2, \bullet}(X)\right)\right) p^{n} q^{d} .
$$




\section{J. Bryan, G. Oberdieck, R. Pandharipande And Q. Yin}

These terms have been calculated in Steps 5 and 6 of the proof of Theorem 1.6(ii). Summing, we obtain

$$
K(p, q)^{4} \cdot\left(-3 \wp(p, q)-\frac{1}{4}+6 \sum_{d \geqslant 1} \sum_{k \mid d} k\left(2 q^{2 d}-q^{d}\right)\right)
$$

where

$$
\wp(p, q)=\frac{1}{12}+\frac{p}{(1-p)^{2}}+\sum_{d \geqslant 1} \sum_{k \mid d} k\left(p^{k}-2+p^{-k}\right) q^{d}
$$

is the Weierstrass elliptic function expanded in $p$ and $q$. Rewriting

$$
-\frac{1}{4}+6 \sum_{d \geqslant 1} \sum_{k \mid d} k\left(2 q^{2 d}-q^{d}\right)=-\frac{1}{4}\left(1+24 \sum_{d \geqslant 1} \sum_{k \mid d} k q^{d}-24 \sum_{d \geqslant 1} \sum_{\substack{k \mid d \\ k \text { even }}} k q^{d}\right)=-\frac{1}{4} \vartheta_{D_{4}}(q),
$$

where

$$
\vartheta_{D_{4}}(q)=1+24 \sum_{d \geqslant 1} \sum_{\substack{k \mid d \\ k \text { odd }}} k q^{d}
$$

is the theta function of the $D_{4}$ lattice, we find

$$
\mathrm{DT}_{2}=-K(p, q)^{4} \cdot\left(3 \wp(p, q)+\frac{1}{4} \vartheta_{D_{4}}(q)\right) .
$$

Hence, part (ii) of Corollary* 1.7 follows from Lemma 7.4 below.

LEMMA 7.4. We have

$$
K(p, q)^{4} \cdot\left(3 \wp(p, q)+\frac{1}{4} \vartheta_{D_{4}}(q)\right)=\frac{3}{2} K(p, q)^{4} \wp(p, q)+\frac{3}{8} K\left(p^{2}, q^{2}\right)^{2} .
$$

Proof. The lemma is stated as an equality of formal power series. Since both sides converge for the variables $p=e^{2 \pi i z}$ and $q=e^{2 \pi i \tau}$ with $z \in \mathbb{C}$ and $\tau \in \mathbb{H}$, we may work with the actual functions $K(z, \tau), \wp(z, \tau)$, and $\vartheta_{D_{4}}(\tau)$.

The statement of the lemma is then equivalent to

$$
\varphi(z, \tau)=\frac{K(2 z, 2 \tau)^{2}}{K(z, \tau)^{4}}-4 \wp(z, \tau)=\frac{2}{3} \vartheta_{D_{4}}(\tau) .
$$

From the definition of $K(z, \tau)$, we obtain

$$
K(z+\lambda \tau+\mu, \tau)=(-1)^{\lambda+\mu} q^{-\lambda^{2} / 2} p^{-\lambda} K(z, \tau)
$$

for all $\lambda, \mu \in \mathbb{Z}$. Combined with the double-periodicity of the Weierstrass $\wp$-function, this implies $\varphi(z+\lambda \tau+\mu, \tau)=\varphi(z, \tau)$ for all $\lambda, \mu \in \mathbb{Z}$. Since

$$
K(z, \tau)=2 \pi i z+O\left(z^{3}\right) \text { and } \wp(z, \tau)=\frac{1}{(2 \pi i z)^{2}}+O(1),
$$

the function $\varphi(z, \tau)$ has no pole at $z=0$. Because the only zero of $K(z, \tau)$ and $\vartheta_{1}(z, \tau)$ and the only pole of $\wp(z, \tau)$ in the fundamental region are at $z=0$, the function $\varphi(z, \tau)$ is entire. By double-periodicity, $\varphi(z, \tau)$ is hence a constant depending only on $\tau$.

We evaluate $\varphi(z, \tau)$ at $z=1 / 2$. We have $\wp(1 / 2, \tau)=-\frac{1}{6} \vartheta_{D_{4}}(\tau)$. Since $K(1 / 2, \tau) \neq 0$ but $K(1, \tau)=0$, this shows

$$
\varphi(z, \tau)=\varphi\left(\frac{1}{2}, \tau\right)=-4 \cdot\left(-\frac{1}{6} \vartheta_{D_{4}}(\tau)\right)=\frac{2}{3} \vartheta_{D_{4}}(\tau) .
$$




\section{Curve COUnting on ABElian SURFACES AND THREefoldS}

\subsection{Discussion of Conjecture D}

The phenomenon proposed by Conjecture D is parallel to the phenomenon exhibited by the Donaldson-Thomas invariants of toric Calabi-Yau threefolds. In the case of toric Calabi-Yau threefolds, the only subschemes which contribute to the DT invariants are the torus-fixed subschemes, namely those which are locally given by monomial ideals. The value of the Behrend function at such a subscheme $Z$ is given by $\pm(-1)^{n}$, where $n=\chi\left(\mathcal{O}_{Z}\right)$ and the overall sign depends only on the 1-dimensional component of $Z$ (and not on the embedded points) [MNOP06].

One route to prove Conjecture D would be to show the following two properties:

(i) The motivic methods of the previous section are compatible with the Behrend function; specifically, the group actions defined on the various substrata of $\operatorname{Hilb}(X) / X$ respect the Behrend function.

(ii) The value of the Behrend function at a subscheme $Z$ which is formally locally given by monomial ideals is given by $\pm(-1)^{n}$, where the overall sign is positive if $Z$ contains a diagonal curve and negative if $Z$ contains a vertical curve.

\section{Gromov-Witten theory}

\subsection{Overview}

Let $X$ be an abelian threefold, let $g \geqslant 2$ be the genus, and let $\beta \in H_{2}(X, \mathbb{Z})$ be a curve class of type $\left(d_{1}, d_{2}, d_{3}\right)$ with $d_{1}, d_{2}>0$.

In Section 8.2, we define a virtual fundamental class on the quotient stack $\bar{M}_{g}(X, \beta) / X$. The degree of the virtual class is the quotient Gromov-Witten invariant of $X$.

The reduced Gromov-Witten invariants of $X$ are defined by integration against the 3-reduced virtual class (defined in Section 2.4) on the moduli space $\bar{M}_{g, n}(X, \beta)$. In Section 8.3, we prove that these invariants are fully determined by the quotient Gromov-Witten invariants and classical intersections.

In Section 8.4, we relate the quotient invariants in genus 3 to the lattice counts of Section 2.3. We also prove the crucial Lemma 8.6 needed in Section 7. In Section 8.5, we use Jacobi form techniques to show that Conjectures $\mathrm{B}$ and $\mathrm{C}$ are consistent with Theorem 1.6.

Finally, we extend Conjecture C to all curve classes in Section 8.6.

\subsection{Quotient invariants}

Since $g \geqslant 2$, the threefold $X$ acts on $\bar{M}_{g}(X, \beta)$ with finite stabilizers. Let

$$
q: \bar{M}_{g}(X, \beta) \rightarrow \bar{M}_{g}(X, \beta) / X
$$

be the quotient map. Let $0_{X} \in X$ be the identity element, let ev: $\bar{M}_{g, 1}(X, \beta) \rightarrow X$ be the evaluation map, let $\psi_{1}$ be the first Chern class of the cotangent line $L_{1} \rightarrow \bar{M}_{g, 1}(X, \beta)$, and let $\pi: \bar{M}_{g, 1}(X, \beta) \rightarrow \bar{M}_{g}(X, \beta)$ be the forgetful map.

We define the reduced virtual class on $\bar{M}_{g}(X, \beta) / X$ by

$$
\left[\bar{M}_{g}(X, \beta) / X\right]^{\mathrm{red}}=\frac{1}{2 g-2}(q \circ \pi)_{*}\left(\left(\mathrm{ev}^{-1}\left(0_{X}\right) \cup \psi_{1}\right) \cap\left[\bar{M}_{g, 1}(X, \beta)\right]^{\mathrm{red}}\right) .
$$

The definition is justified by the following lemma.

Lemma 8.1. Let $p: \bar{M}_{g, n}(X, \beta) \rightarrow \bar{M}_{g}(X, \beta) / X$ be the composition of the forgetful map with 


\section{J. Bryan, G. Oberdieck, R. Pandharipande and Q. Yin}

the quotient map. Then,

$$
p^{*}\left[\bar{M}_{g}(X, \beta) / X\right]^{\mathrm{red}}=\left[\bar{M}_{g, n}(X, \beta)\right]^{\mathrm{red}} .
$$

Proof. The map $p$ factors as

$$
p: \bar{M}_{g, n}(X, \beta) \stackrel{p^{\prime}}{\longrightarrow} \bar{M}_{g}(X, \beta) \stackrel{q}{\longrightarrow} \bar{M}_{g}(X, \beta) / X,
$$

where $p^{\prime}$ is the forgetful map and $q$ is the quotient map (8.1). Since we have

$$
p^{* *}\left[\bar{M}_{g}(X, \beta)\right]^{\mathrm{red}}=\left[\bar{M}_{g, n}(X, \beta)\right]^{\mathrm{red}}
$$

it is enough to prove

$$
q^{*}\left[\bar{M}_{g}(X, \beta) / X\right]^{\mathrm{red}}=\left[\bar{M}_{g}(X, \beta)\right]^{\mathrm{red}} .
$$

Consider the product decomposition

$$
\bar{M}_{g, 1}(X, \beta)=\bar{M}_{g, 1}^{0}(X, \beta) \times X,
$$

where $\bar{M}_{g, 1}^{0}(X, \beta)=\mathrm{ev}^{-1}\left(0_{X}\right)$. Under the decomposition (8.2), write

$$
q^{\prime}: \bar{M}_{g, 1}(X, \beta) \rightarrow \bar{M}_{g, 1}^{0}(X, \beta)
$$

for the projection to the first factor. Since the obstruction theory of $\bar{M}_{g, 1}(X, \beta)$ is $X$-equivariant, we have

$$
\psi_{1} \cap\left[\bar{M}_{g, 1}(X, \beta)\right]^{\mathrm{red}}=q^{\prime *} \alpha
$$

for some class $\alpha$ on $\bar{M}_{g, 1}^{0}(X, \beta)$.

Consider the inclusion

$$
\iota: \bar{M}_{g, 1}^{0}(X, \beta) \rightarrow \bar{M}_{g, 1}(X, \beta)
$$

defined by $\bar{M}_{g, 1}^{0}(X, \beta) \times 0_{X}$ under (8.2) and the fiber diagram

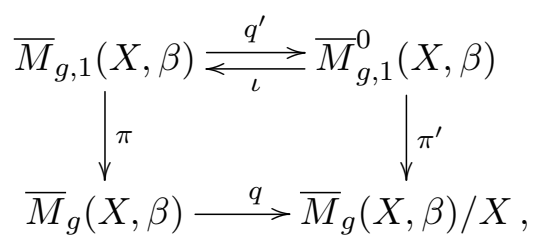

where $\pi^{\prime}$ is the map induced by $\pi$. Then

$$
\begin{aligned}
(2 g-2) q^{*}\left[\bar{M}_{g}(X, \beta) / X\right]^{\mathrm{red}} & =q^{*} \pi_{*}^{\prime} q_{*}^{\prime}\left(\left(\mathrm{ev}^{-1}\left(0_{X}\right) \cup \psi_{1}\right) \cap\left[\bar{M}_{g, 1}(X, \beta)\right]^{\mathrm{red}}\right) \\
& =\pi_{*} q^{\prime *} q_{*}^{\prime}\left(\left(\mathrm{ev}^{-1}\left(0_{X}\right) \cup \psi_{1}\right) \cap\left[\bar{M}_{g, 1}(X, \beta)\right]^{\mathrm{red}}\right) \\
& =\pi_{*} q^{\prime *} \iota^{*}\left(\psi_{1} \cap\left[\bar{M}_{g, 1}(X, \beta)\right]^{\mathrm{red}}\right) \\
& =\pi_{*} q^{\prime *} \alpha \\
& =\pi_{*}\left(\psi_{1} \cap\left[\bar{M}_{g, 1}(X, \beta)\right]^{\mathrm{red}}\right) .
\end{aligned}
$$

The lemma now follows directly from the dilaton equation.

We define the quotient Gromov-Witten invariants of $X$ by

$$
\mathrm{N}_{g, \beta}=\int_{\left[\bar{M}_{g}(X, \beta) / X\right]^{\mathrm{red}}} 1 .
$$




\section{CURVE COUNTING ON ABELIAN SURFACES AND THREEFOLDS}

\subsection{Reduced Gromov-Witten invariants}

Let $g \geqslant 2$, and let $\beta$ be a curve class of type $\left(d_{1}, d_{2}, d_{3}\right)$ with $d_{1}, d_{2}>0$. Let $\left[\bar{M}_{g, n}(X, \beta)\right]^{\text {red }}$ be the 3 -reduced virtual class on the moduli space $\bar{M}_{g, n}(X, \beta)$ constructed in Section 2.4. The reduced Gromov-Witten invariants of $X$ are defined by

$$
\left\langle\tau_{a_{1}}\left(\gamma_{1}\right) \cdots \tau_{a_{n}}\left(\gamma_{n}\right)\right\rangle_{g, \beta}^{X, \mathrm{red}}=\int_{\left[\bar{M}_{g, n}(X, \beta)\right]^{\mathrm{red}}} \prod_{i=1}^{n} \operatorname{ev}_{i}^{*}\left(\gamma_{i}\right) \cup \psi_{i}^{a_{i}}
$$

for $\gamma_{1}, \ldots, \gamma_{n} \in H^{*}(X, \mathbb{Q})$ and $a_{1}, \ldots, a_{n} \geqslant 0$.

By the definition of the virtual class $\left[\bar{M}_{g}(X, \beta) / X\right]^{\text {red }}$ and the quotient Gromov-Witten invariants (8.3), we have

$$
\mathrm{N}_{g, \beta}=\frac{1}{2 g-2} \cdot\left\langle\tau_{1}(\mathrm{p})\right\rangle_{g, \beta}^{X, \text { red }},
$$

where $\mathrm{p} \in H^{6}(X, \mathbb{Z})$ is the class of a point. The invariants $\mathrm{N}_{g, \beta}$ will be shown to determine all reduced Gromov-Witten invariants (8.4) of $X$.

We first determine all primary Gromov-Witten invariants in terms of $\mathrm{N}_{g, \beta}$. Consider the translation action

$$
t: X^{n+1} \rightarrow X, \quad\left(a, x_{1}, \ldots, x_{n}\right) \mapsto\left(x_{1}-a, \ldots, x_{n}-a\right) .
$$

Lemma 8.2. For $\gamma_{1}, \ldots, \gamma_{n} \in H^{*}(X, \mathbb{Q})$,

$$
\left\langle\tau_{0}\left(\gamma_{1}\right) \cdots \tau_{0}\left(\gamma_{n}\right)\right\rangle_{g, \beta}^{X, \text { red }}=\mathrm{N}_{g, \beta} \cdot \int_{t_{*}([X] \otimes \beta \otimes n)} \gamma_{1} \otimes \cdots \otimes \gamma_{n} .
$$

Proof. Let $\gamma_{1}, \ldots, \gamma_{n} \in H^{*}(X, \mathbb{Q})$ be homogeneous classes. We may assume that the dimension constraint

$$
\sum_{i=1}^{n} \operatorname{deg}\left(\gamma_{i}\right)=2(3+n)
$$

holds, where $\operatorname{deg}(\cdot)$ denotes the real degree of a class in $X$; otherwise, both sides of (8.6) vanish.

For every $k$, let

$$
\pi_{k}: \bar{M}_{g, k}(X, \beta) \rightarrow \bar{M}_{g}(X) / X
$$

be the composition of the map that forgets all markings with the quotient map. By Lemma 8.1, we have

$$
\left[\bar{M}_{g, n}(X, \beta)\right]^{\mathrm{red}}=\pi_{n}^{*}\left[\bar{M}_{g}(X, \beta) / X\right]^{\mathrm{red}}
$$

hence, by the push-pull formula, we have

$$
\left\langle\tau_{0}\left(\gamma_{1}\right) \cdots \tau_{0}\left(\gamma_{n}\right)\right\rangle_{g, \beta}^{X, \text { red }}=\int_{\left[\bar{M}_{g}(X, \beta) / X\right]^{\mathrm{red}}} \pi_{n *}\left(\prod_{i} \operatorname{ev}_{i}^{*}\left(\gamma_{i}\right)\right) .
$$

Since the map $\pi_{n}$ is of relative dimension $3+n$, the cohomology class $\pi_{n *}\left(\prod_{i} \operatorname{ev}_{i}^{*}\left(\gamma_{i}\right)\right)$ has degree 0 . To proceed, we evaluate $\prod_{i} \mathrm{ev}_{i}^{*}\left(\gamma_{i}\right)$ on the fibers of $\pi_{n}$.

Let $f: C \rightarrow X$ be a stable map of genus $g$ and class $\beta$, let $[f] \in \bar{M}_{g}(X, \beta) / X$ be the associated point, and let $F$ be the (stack) fiber of $\pi_{n}$ over $[f]$.

By the definition of $\bar{M}_{g}(X, \beta) / X$ as a quotient stack [Rom05], we may identify

$$
X=\pi_{0}^{-1}([f]),
$$

where the induced map $X \rightarrow \bar{M}_{g}(X, \beta)$ is $x \mapsto(f-x)$. 


\section{J. Bryan, G. Oberdieck, R. Pandharipande and Q. Yin}

Under (8.7), let $b_{0}: F \rightarrow X$ be the map which forgets all markings. For $i \in\{1, \ldots, n\}$, let $b_{i}: F \rightarrow C$ be the map which forgets all except the $i$ th marking. The induced map

$$
b=\left(b_{0}, \ldots, b_{n}\right): F \rightarrow X \times C^{n}
$$

is birational on components.

The evaluation map ev: $F \rightarrow X^{n}$ factors as

$$
F \stackrel{b}{\rightarrow} X \times C^{n} \stackrel{(\mathrm{id}, f, \ldots, f)}{\longrightarrow} X^{n+1} \stackrel{t}{\rightarrow} X^{n},
$$

where $t$ is the translation map (8.5). We find

$$
\begin{aligned}
\int_{F} \mathrm{ev}_{1}^{*}\left(\gamma_{1}\right) \cdots \mathrm{ev}_{n}^{*}\left(\gamma_{n}\right) & =\int_{\mathrm{ev}_{*}[F]} \gamma_{1} \otimes \cdots \otimes \gamma_{n} \\
& =\int_{t_{*}\left(\mathrm{id}, f^{n}\right)_{*}\left([X] \otimes[C]^{\otimes n}\right)} \gamma_{1} \otimes \cdots \otimes \gamma_{n} \\
& =\int_{t_{*}\left([X] \otimes \beta^{\otimes n}\right)} \gamma_{1} \otimes \cdots \otimes \gamma_{n} .
\end{aligned}
$$

Since this depends only on $\beta$ and the $\gamma_{i}$, we conclude

$$
\pi_{n *}\left(\prod_{i} \mathrm{ev}_{i}^{*}\left(\gamma_{i}\right)\right)=\left(\int_{t_{*}\left([X] \otimes \beta^{n}\right)} \gamma_{1} \otimes \cdots \otimes \gamma_{n}\right) \cdot 1
$$

The claim of the lemma follows.

We state the abelian vanishing relation for abelian threefolds. Consider the map defined by

$$
p: X^{n} \rightarrow X^{n-1}, \quad\left(x_{1}, \ldots, x_{n}\right) \mapsto\left(x_{2}-x_{1}, \ldots, x_{n}-x_{1}\right) .
$$

Lemma 8.3. Let $\gamma \in H^{*}\left(X^{n-1}, \mathbb{Q}\right)$, and let $a_{1}, \ldots, a_{n} \geqslant 0$. For any $\gamma_{1} \in H^{*}(X, \mathbb{Q})$ of degree $\operatorname{deg}\left(\gamma_{1}\right) \leqslant 5$, we have

$$
\int_{\left[\bar{M}_{g, n}(X, \beta)\right]^{\mathrm{red}}} \operatorname{ev}_{1}^{*}\left(\gamma_{1}\right) \cup \mathrm{ev}^{*} p^{*}(\gamma) \cup \prod_{i} \psi_{i}^{a_{i}}=0 .
$$

The proof is identical to the proof of Lemma 4.2.

Proposition 8.4. The full reduced descendent Gromov-Witten theory of $X$ in genus $g$ and class $\beta$ is determined from $\mathrm{N}_{g, \beta}$ by the following operations:

(i) the string, dilaton, and divisor equations,

(ii) the abelian vanishing relation of Lemma 8.3,

(iii) the evaluation by Lemma 8.2 of primary invariants,

(iv) the evaluation $\left\langle\tau_{1}(\mathrm{p})\right\rangle_{g, \beta}^{X, \text { red }}=(2 g-2) \cdot \mathrm{N}_{g, \beta}$.

Proof. Let $\gamma_{1}, \ldots, \gamma_{n} \in H^{*}(X, \mathbb{Q})$ be homogeneous classes. We must determine the GromovWitten invariant

$$
\left\langle\tau_{a_{1}}\left(\gamma_{1}\right) \cdots \tau_{a_{n}}\left(\gamma_{n}\right)\right\rangle_{g, \beta}^{X, \text { red }}
$$

for $a_{1}, \ldots, a_{n} \geqslant 0$. We may assume the dimension constraint

$$
\sum_{i=1}^{n} \operatorname{deg}\left(\gamma_{i}\right)+2 a_{i}=2(3+n),
$$




\section{CURVE COUNTING ON ABELIAN SURFACES AND THREEFOLDS}

where $\operatorname{deg}(\cdot)$ is the real degree of a class in $X$. In particular, we have $n \geqslant 1$.

We proceed by induction on $n$. In case $n=1$, the insertion must be

$$
\tau_{1}(\mathrm{p}), \quad \tau_{2}(\gamma), \quad \tau_{3}\left(\gamma^{\prime}\right), \quad \text { or } \tau_{4}\left(\gamma^{\prime \prime}\right)
$$

for classes $\gamma, \gamma^{\prime}, \gamma^{\prime \prime}$ of degree $4,2,0$, respectively. The case $\tau_{1}(\mathrm{p})$ follows from (iv). The cases $\tau_{2}(\gamma)$, $\tau_{3}\left(\gamma^{\prime}\right)$, and $\tau_{4}\left(\gamma^{\prime \prime}\right)$ all vanish by the abelian vanishing relation (ii).

Suppose $n>1$ and assume that the proposition is true for all $n^{\prime}<n$. If $a_{i}=0$ for all $i$, the statement follows from the evaluation (iii). Hence, we may assume $a_{1}>0$. If $\operatorname{deg}\left(\gamma_{1}\right)<6$, we first apply the vanishing of Lemma 8.3 for $\gamma_{1}$ and $\gamma=\gamma_{2} \otimes \cdots \otimes \gamma_{n}$. We find that (8.8) can be expressed as a sum of series

$$
\pm\left\langle\tau_{a_{1}}\left(\gamma_{1} \cup \delta\right) \tau_{a_{2}}\left(\gamma_{2}^{\prime}\right) \cdots \tau_{a_{n}}\left(\gamma_{n}^{\prime}\right)\right\rangle_{g, \beta}^{X, \text { red }}
$$

for homogeneous classes $\delta, \gamma_{2}^{\prime}, \ldots, \gamma_{n}^{\prime} \in H^{*}(X, \mathbb{Q})$ with $\operatorname{deg}(\delta) \geqslant 1$. The above relation increases the degree of $\gamma_{1}$. By induction on $\operatorname{deg}\left(\gamma_{1}\right)$, we may assume $\operatorname{deg}\left(\gamma_{1}\right)=6$.

By the dimension constraint (8.9), we have

$$
\sum_{i=2}^{n} \operatorname{deg}\left(\gamma_{i}\right)+2 a_{i}=2\left(n-a_{1}\right)
$$

hence, there exists a $k \in\{2, \ldots, n\}$ such that $\operatorname{deg}\left(\gamma_{k}\right)+2 a_{k} \leqslant 2$.

If $a_{k}=1$, then $\operatorname{deg}\left(\gamma_{k}\right)=0$ and we use the dilaton equation. If $a_{k}=0$ and $\operatorname{deg}\left(\gamma_{i}\right) \in\{0,1\}$, we use the string equation. If $a_{k}=0$ and $\operatorname{deg}\left(\gamma_{i}\right)=2$, we use the divisor equation. In each case, we reduce to Gromov-Witten invariants with fewer than $n$ marked points. The proof of the proposition now follows from the induction hypothesis.

In (3.3), we defined quotient invariants $\mathbf{N}_{g,\left(d_{1}, d_{2}\right)}^{\mathrm{Q}}$ counting genus $g$ curves on an abelian surface $A$ in class of type $\left(d_{1}, d_{2}\right)$, with $g \geqslant 2$ and $d_{1}, d_{2}>0$. By trading the FLS condition for insertions, moving the calculation to the threefold $A \times E$ via the $k=2$ case of Section 2.5, and by the evaluation of Lemma 8.2, one obtains

$$
\mathrm{N}_{g,\left(d_{1}, d_{2}\right)}^{\mathrm{Q}}=\mathrm{N}_{g,\left(d_{1}, d_{2}, 0\right)} \text {. }
$$

Hence, the quotient invariants of abelian surfaces agree with the degenerate case of the quotient invariants of abelian threefolds.

\subsection{Genus 3 counts}

We determine the genus 3 invariants of $X$ using the lattice method of Section 2.3. The strategy is similar to the proof of Lemma 3.1.

LEMmA 8.5. For all $d_{1}, d_{2}, d_{3}>0$, we have $\mathrm{N}_{3,\left(d_{1}, d_{2}, d_{3}\right)}=2 \nu\left(d_{1}, d_{2}, d_{3}\right)$.

Proof. Let $\beta$ be a curve class of type $\left(d_{1}, d_{2}, d_{3}\right)$ on a generic abelian threefold $X$. Since $X$ is simple, every genus 3 stable map $f: C \rightarrow X$ in class $\beta$ has a non-singular domain $C$ and induces a polarized isogeny $(\widehat{X}, \widehat{\beta}) \rightarrow(J, \theta)$.

Conversely, every simple principally polarized abelian threefold $(B, \theta)$ is the Jacobian of a unique non-singular genus 3 curve $C$. Hence, each polarized isogeny $(\widehat{X}, \widehat{\beta}) \rightarrow(B, \theta)$ induces a map

$$
f: C \stackrel{\text { aj }}{\rightarrow} B \rightarrow X
$$




\section{J. Bryan, G. Oberdieck, R. Pandharipande And Q. Yin}

However, for a generic abelian threefold $X$, we have $\operatorname{Aut}(C)=\{1\}$ and $\operatorname{Aut}(X)=\{ \pm 1\}$. The composition

$$
f^{-}=(-1) \circ f: C \rightarrow X
$$

is not translation equivalent to $f$, and the given polarized isogeny corresponds to two genus 3 stable maps up to translation. (This fact was overlooked in [LS02a].)

The argument in the proof of Lemma 3.1 also shows that $X$ acts freely on $\bar{M}_{3}(X, \beta)$. The only point to verify is that given a non-singular genus 3 curve $C$ and the Abel-Jacobi map aj: $C \rightarrow J$, the only element in $J$ fixing aj $(C)$ is $0_{J}$. For this, we consider the map

$$
\operatorname{Sym}^{2}\left(\text { aj) }: \operatorname{Sym}^{2}(C) \rightarrow J\right. \text {. }
$$

The image of $\operatorname{Sym}^{2}(C)$ is a theta divisor and is only fixed by $0_{J} \in J$. Then, if a point $a \in J$ fixes aj $(C)$, it must also fix the image of $\operatorname{Sym}^{2}(C)$ under $\operatorname{Sym}^{2}($ aj). Hence $a=0 \mathrm{~J}$.

It follows that $\bar{M}_{3}(X, \beta) / X$ is a set of $2 \nu\left(d_{1}, d_{2}, d_{3}\right)$ isolated reduced points.

By Lemma 3.1, Theorem 3.2, and Lemma 8.5,

$$
\mathrm{N}_{3,\left(1, d, d^{\prime}\right)}=2 \mathrm{~N}_{2,\left(d, d^{\prime}\right)}^{\mathrm{Q}}=2 \sum_{k \mid \operatorname{gcd}\left(d, d^{\prime}\right)} \sum_{m \mid d d^{\prime} / k^{2}} k^{3} m .
$$

The right-hand side of (8.10) matches precisely the genus 3 predictions of Conjectures B and C.

Further, the lattice method can be adjusted to count diagonal curves in the $X=A \times E$ setting. Let $A, E$, and $\left(\beta_{d^{\prime}}, d\right)$ be as in Section 7 . Recall that an irreducible curve $C \subset X$ is diagonal if both projections $p_{A}: C \rightarrow A$ and $p_{E}: C \rightarrow E$ are of non-zero degree.

LEMma 8.6. For even $d$, there are $12 \sigma(d / 2)=12 \sum_{k \mid d / 2} k$ isolated diagonal curves in class $\left(\beta_{2}, d\right)$ up to translation. All diagonal curves are non-singular of genus 3. The translation action of $A \times E$ on the diagonal curves is free.

Proof. Let $C$ be a diagonal curve in class $\left(\beta_{2}, d\right)$. Since $\beta_{2}$ is irreducible, the projection $p_{A}: C \rightarrow A$ is generically injective. The image $C_{0}=p_{A}(C) \subset A$ is either a non-singular genus 3 curve or a nodal genus 2 curve. We claim that the latter does not happen.

Suppose that it does, and let $q: \widetilde{C} \rightarrow C$ be the normalization map. Then $p_{A} \circ q: \widetilde{C} \rightarrow A$ factors through an isogeny $J(\widetilde{C}) \rightarrow A$, where $J(\widetilde{C})$ is the Jacobian of the genus 2 curve $\widetilde{C}$. We also know that $p_{E} \circ q: \widetilde{C} \rightarrow E$ factors through $J(\widetilde{C}) \rightarrow E$, which is surjective since $d>0$. This contradicts the assumption that $A$ is simple.

Hence, $C_{0}$ is non-singular of genus 3 and so is $C$. As before, every such $C$ induces a polarized isogeny

$$
\left(\widehat{A \times E}, \widehat{\left(\beta_{2}, d\right)}\right) \rightarrow(J, \theta) .
$$

Conversely, every principally polarized abelian threefold $(B, \theta)$ is

- either the Jacobian of a unique non-singular genus 3 curve,

- or the product of a principally polarized abelian surface and an elliptic curve, with the product polarization.

Given $\left(\beta_{2}, d\right)$ of type $(1,2, d)$, we know exactly which maximal totally isotropic subgroups of $\operatorname{Ker}\left(\phi_{\widehat{\left(\beta_{2}, d\right)}}\right) \cong(\mathbb{Z} / 2 \times \mathbb{Z} / d)^{2}$ correspond to polarized isogenies

$$
\left(\widehat{A \times E}, \widehat{\left(\beta_{2}, d\right)}\right) \rightarrow(B, \theta)
$$




\section{Curve Counting on ABELIAN SuRfaces And THREefolds}

to Jacobians $(B, \theta)$. They are precisely the subgroups not of the form

$$
G_{1} \times G_{2} \text { with } G_{1}<(\mathbb{Z} / 2)^{2}, \quad G_{2}<(\mathbb{Z} / d)^{2} .
$$

In particular, $d$ must be even for these subgroups to exist. In terms of (2.2), there are the following two possibilities:

(i) $K=\mathbb{Z} / 2 k$ for some $k \mid d / 2$, generated by

$$
\left(1, \frac{d}{2 k}\right) \in \mathbb{Z} / 2 \times \mathbb{Z} / d
$$

together with an arbitrary element in $\operatorname{Hom}^{\mathrm{sym}}(K, \widehat{K})$,

(ii) $K=\mathbb{Z} / 2 \times \mathbb{Z} / 2 k$ for some $k \mid d / 2$, generated by

$$
(1,0),\left(0, \frac{d}{2 k}\right) \in \mathbb{Z} / 2 \times \mathbb{Z} / d
$$

together with a non-diagonal element in $\operatorname{Hom}^{\mathrm{sym}}(K, \widehat{K})$.

Summing the number of subgroups of types (i) and (ii), we find

$$
\sum_{k \mid d / 2} 2 k+\sum_{k \mid d / 2} 2 \cdot 2 k=6 \sum_{k \mid d / 2} k
$$

polarized isogenies to Jacobians. We claim that each of the isogenies corresponds to two diagonal curves up to translation.

We have seen that a diagonal curve $C \subset A \times E$ is isomorphic to its image $C_{0} \subset A$. By [BL04, Section 10.8(1)], every non-singular genus 3 curve $C^{\prime} \subset A$ in class $\beta_{2}$ admits a double cover to an elliptic curve $E^{\prime}$. In particular, the Jacobian $J\left(C^{\prime}\right)$ is isogenous to $A \times E^{\prime}$. Hence,

$$
\mathbb{Z} / 2 \subset \operatorname{Aut}\left(C_{0}\right)=\operatorname{Aut}(C) .
$$

On the other hand, we have generically $\operatorname{Aut}(A \times E)=\mathbb{Z} / 2 \times \mathbb{Z} / 2$. Since the Jacobian $J$ of $C$ is isogenous to $A \times E$, we also have

$$
\operatorname{Aut}(J) \subset \mathbb{Z} / 2 \times \mathbb{Z} / 2 .
$$

A strong form of the Torelli theorem (see [BL04, Section 11.12(19)]) says

$$
\operatorname{Aut}(C)= \begin{cases}\operatorname{Aut}(J, \theta) & \text { if } C \text { is hyperelliptic } \\ \operatorname{Aut}(J, \theta) /\{ \pm 1\} & \text { if } C \text { is not hyperelliptic }\end{cases}
$$

In our case, this means

$$
\operatorname{Aut}(C)= \begin{cases}\mathbb{Z} / 2 \times \mathbb{Z} / 2 & \text { if } C \text { is hyperelliptic, } \\ \mathbb{Z} / 2 & \text { if } C \text { is not hyperelliptic } .\end{cases}
$$

To see that $C$ is generically not hyperelliptic, recall from Section 6.3 that up to translation there are three ${ }^{9}$ hyperelliptic genus 3 curves

$$
C_{1}^{\prime}, C_{2}^{\prime}, C_{3}^{\prime} \subset A
$$

in class $\beta_{2}$ with $\mathbb{Z} / 2$-stabilizers. For $i=1,2,3$, the Jacobian $J\left(C_{i}^{\prime}\right)$ is isogenous to $A \times E_{i}^{\prime}$ for some $E_{i}^{\prime}$. Hence, by taking $E$ non-isogenous to $E_{1}^{\prime}, E_{2}^{\prime}, E_{3}^{\prime}$, we find that the diagonal curve $C \subset A \times E$ is not isomorphic to $C_{1}^{\prime}, C_{2}^{\prime}, C_{3}^{\prime}$.

\footnotetext{
${ }^{9}$ Each corresponds to a degree 2 polarized isogeny $\left(A, \beta_{2}\right) \rightarrow(B, \theta)$, with $B$ the Jacobian of a genus 2 curve. The hyperelliptic curve is obtained by taking the preimage of the genus 2 curve.
} 


\section{J. Bryan, G. Oberdieck, R. Pandharipande and Q. Yin}

To conclude, we have $\operatorname{Aut}(C)=\mathbb{Z} / 2$ and $\operatorname{Aut}(J)=\operatorname{Aut}(A \times E)=\mathbb{Z} / 2 \times \mathbb{Z} / 2$. Therefore, each polarized isogeny (8.11) gives two diagonal curves up to translation. We find in total

$$
2 \cdot 6 \sum_{k \mid d / 2} k=12 \sum_{k \mid d / 2} k
$$

diagonal curves up to translation. The proof that $A \times E$ acts freely is identical to the one given in the proof of Lemma 8.5.

\subsection{Consistency check of Conjectures B and C}

Conjecture $\mathrm{C}$ expresses the invariants $\mathrm{N}_{g,\left(1, d^{\prime}, d\right)}$ in terms of the invariants $\mathrm{N}_{g,(1,1, d)}$. By Conjecture B, we obtain a prediction for the Donaldson-Thomas invariants of type $(1,2, d)$ in terms of those of $(1,1, d)$. We show here that these predictions match the calculations of Theorem 1.6.

For $d \geqslant 0$, let $f_{d}(p), g_{d}(p) \in \mathbb{Q}((p))$ be the unique Laurent series with

$$
\sum_{d \geqslant 0} f_{d}(p) q^{d}=K(z, \tau)^{2}, \quad \sum_{d \geqslant 0} g_{d}(p) q^{d}=\frac{3}{2} K(z, \tau)^{4} \wp(z, \tau)+\frac{3}{8} K(2 z, 2 \tau)^{2}
$$

under the variable change $p=e^{2 \pi i z}$ and $q=e^{2 \pi i \tau}$. The functions on the right-hand side of (8.12) are exactly the negative of the functions appearing in Corollary* 1.7. The following lemma shows that Corollary* 1.7 is consistent with Conjectures B and C.

LEMMA 8.7. We have

$$
g_{d}(p)= \begin{cases}f_{2 d}(p) & \text { if } d \text { is odd } \\ f_{2 d}(p)+\frac{1}{2} f_{d / 2}\left(p^{2}\right) & \text { if } d \text { is even }\end{cases}
$$

Proof. We use basic results from the theory of Jacobi forms [EZ85]. We will work with the actual variables $p=e^{2 \pi i z}$ and $q=e^{2 \pi i \tau}$, where $z \in \mathbb{C}$ and $\tau \in \mathbb{H}$.

Let $\varphi_{-2,1}(z, \tau)$ be the weight -2 , index 1 generator of the ring of weak Jacobi forms defined in [EZ85, Section 9]. We have the basic identity

$$
\varphi_{-2,1}(z, \tau)=K(z, \tau)^{2}
$$

see, for example, [DMZ12, equation (4.29)]. Applying the Hecke operator $\left.\right|_{-2,1} V_{2}$ defined in [EZ85, Section 4], we obtain the weak weight -2 , index 2 Jacobi form

$$
\left(\left.\varphi_{-2,1}\right|_{-2,1} V_{2}\right)(z, \tau)=\sum_{d \geqslant 0}\left(f_{2 d}(p)+\frac{f_{d / 2}\left(p^{2}\right)}{2^{3}}\right) q^{d}
$$

where $f_{a}(p)=0$ whenever $a$ is fractional. Using [EZ85, Theorem 9.3] and comparing the first coefficients, we find

$$
\sum_{d \geqslant 0}\left(f_{2 d}(p)+\frac{f_{d / 2}\left(p^{2}\right)}{2^{3}}\right) q^{d}=\frac{3}{2} K(z, \tau)^{4} \wp(z, \tau)
$$


We conclude

$$
\begin{aligned}
\sum_{d \geqslant 0}\left(f_{2 d}(p)+\frac{f_{d / 2}\left(p^{2}\right)}{2}\right) q^{d} & =\frac{3}{2} K(z, \tau)^{4} \wp(z, \tau)+\sum_{d \geqslant 0} \frac{3}{8} f_{d / 2}\left(p^{2}\right) q^{d} \\
& =\frac{3}{2} K(z, \tau)^{4} \wp(z, \tau)+\frac{3}{8} K(2 z, 2 \tau)^{2} \\
& =\sum_{d \geqslant 0} g_{d}(p) q^{d}
\end{aligned}
$$

\subsection{A formula for imprimitive classes}

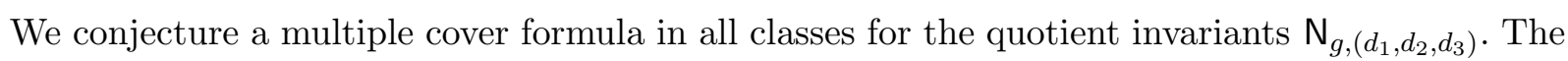
shape of the formula already appeared in the physics approach of [MMS99]. However, [MMS99] does not match the invariants $\mathrm{N}_{g, \beta}$ and our formula below is different.

Define the function

$$
\mathrm{n}\left(d_{1}, d_{2}, d_{3}, k\right)=\sum_{\delta} \delta^{2}
$$

where $\delta$ runs over all divisors of

$$
\operatorname{gcd}\left(k, d_{1}, d_{2}, d_{3}, \frac{d_{1} d_{2}}{k}, \frac{d_{1} d_{3}}{k}, \frac{d_{2} d_{3}}{k}, \frac{d_{1} d_{2} d_{3}}{k^{2}}\right) .
$$

Conjecture E. For all $g \geqslant 2, d_{1}, d_{2}>0$, and $d_{3} \geqslant 0$,

$$
\mathrm{N}_{g,\left(d_{1}, d_{2}, d_{3}\right)}=\sum_{k} \mathrm{n}\left(d_{1}, d_{2}, d_{3}, k\right) k^{2 g-3} \mathrm{~N}_{g,\left(1,1, d_{1} d_{2} d_{3} / k^{2}\right)},
$$

where $k$ runs over all divisors of $\operatorname{gcd}\left(d_{1} d_{2}, d_{1} d_{3}, d_{2} d_{3}\right)$ such that $k^{2}$ divides $d_{1} d_{2} d_{3}$.

Recall the quotient Donaldson-Thomas invariants DT $_{n, \beta}$. Assuming deformation invariance, we write

$$
\mathrm{DT}_{n, \beta}=\mathrm{DT}_{n,\left(d_{1}, d_{2}, d_{3}\right)}
$$

if $\beta$ is of type $\left(d_{1}, d_{2}, d_{3}\right)$. The invariants $\mathrm{DT}_{n,\left(d_{1}, d_{2}, d_{3}\right)}$ are defined whenever $n \neq 0$ or if at least two of the $d_{i}$ are positive.

Translating the multiple cover rule of Conjecture E via the conjectural GW/DT correspondence yields the following.

Conjecture E'. Assume $n>0$ or that at least two of the integers $d_{1}, d_{2}, d_{3}$ are positive. Then

$$
\mathrm{DT}_{n,\left(d_{1}, d_{2}, d_{3}\right)}=\sum_{k} \frac{1}{k} \mathrm{n}\left(d_{1}, d_{2}, d_{3}, k\right)(-1)^{n-(n / k)} \mathrm{DT}_{n / k,\left(1,1, d_{1} d_{2} d_{3} / k^{2}\right)},
$$

where $k$ runs over all divisors of $\operatorname{gcd}\left(n, d_{1} d_{2}, d_{1} d_{3}, d_{2} d_{3}\right)$ such that $k^{2}$ divides $d_{1} d_{2} d_{3}$.

While Conjecture E applies only for $d_{1}, d_{2}>0$, we have stated Conjecture $\mathrm{E}^{\prime}$ also for the degenerate cases $(0,0, d)$. Unraveling the definition yields

$$
\mathrm{DT}_{n,(0,0, d)}=\frac{(-1)^{n-1}}{n} \sum_{k \mid \operatorname{gcd}(n, d)} k^{2},
$$

which for $d=0$ is in perfect agreement with [She15] and for $d>0$ is proven in [OS16b]. 


\section{J. Bryan, G. Oberdieck, R. Pandharipande and Q. Yin}

\section{ACKNOWLEDGEMENTS}

We thank B. Bakker, L. Göttsche, S. Katz, M. Kool, A. Klemm, A. Knutsen, A. Kresch, J. Li, D. Maulik, G. Moore, B. Pioline, M. Raum, S. Rose, J. Schmitt, E. Sernesi, J. Shen, V. Shende, and R. Thomas for discussions about counting invariants of abelian surfaces and threefolds. The results here were first presented at the conference Motivic invariants related to $K 3$ and abelian geometries at Humboldt University in February 2015. We thank G. Farkas and the Einstein Stiftung for support in Berlin.

\section{REFERENCES}

Bea99 A. Beauville, Counting rational curves on K3 surfaces, Duke Math. J. 97 (1999), no. 1, 99-108; https://doi.org/10.1215/S0012-7094-99-09704-1.

Beh99 K. Behrend, The product formula for Gromov-Witten invariants, J. Algebraic Geom. 8 (1999), no. $3,529-541$.

BG09 J. Bryan and T. Graber, The crepant resolution conjecture, Algebraic Geometry - Seattle 2005, Part 1, Proc. Sympos. Pure Math., vol. 80 (Amer. Math. Soc., Providence, RI, 2009), 23-42; https://doi.org/10.1090/pspum/080.1/2483931.

BK16 J. Bryan and M. Kool, Donaldson-Thomas invariants of local elliptic surfaces via the topological vertex, 2016, arXiv:1608.07369.

BL04 C. Birkenhake and H. Lange, Complex abelian varieties, 2nd ed., Grundlehren math. Wiss., vol. 302 (Springer-Verlag, Berlin, 2004); https://doi.org/10.1007/978-3-662-06307-1.

BL99 J. Bryan and N.C. Leung, Generating functions for the number of curves on abelian surfaces, Duke Math. J. 99 (1999), no. 2, 311-328; https://doi.org/10.1215/ S0012-7094-99-09911-8.

BL00 - The enumerative geometry of $K 3$ surfaces and modular forms, J. Amer. Math. Soc. 13 (2000), no. 2, 371-410; https://doi.org/10.1090/S0894-0347-00-00326-X.

BO00 S. Bloch and A. Okounkov, The character of the infinite wedge representation, Adv. Math. 149 (2000), no. 1, 1-60; https://doi.org/10.1006/aima.1999.1845.

BO17 P. Borówka and A. Ortega, Hyperelliptic curves on $(1,4)$ polarised abelian surfaces, 2017, arXiv:1708.01270.

Bry15 J. Bryan, The Donaldson-Thomas theory of $K 3 \times E$ via the topological vertex, in Geometry of moduli, Abel Symp., to appear, arXiv:1504.02920.

BS17 P. Borówka and G.K. Sankaran, Hyperelliptic genus 4 curves on abelian surfaces, Proc. Amer. Math. Soc. 145 (2017), no. 12, 5023-5034.

But94 L. M. Butler, Subgroup lattices and symmetric functions, Mem. Amer. Math. Soc. 112, no. 539 (Amer. Math. Soc., Provindence, RI, 1994); https://doi.org/10.1090/memo/0539.

Cha85 K. Chandrasekharan, Elliptic functions, Grundlehren math. Wiss., vol. 281 (Springer-Verlag, Berlin, 1985); https://doi.org/10.1007/978-3-642-52244-4.

Deb99 O. Debarre, On the Euler characteristic of generalized Kummer varieties, Amer. J. Math. 121 (1999), no. 3, 577-586; https://doi.org/10.1353/ajm.1999.0018.

Deb05_ Complex tori and abelian varieties, SMF/AMS Texts and Monographs, vol. 11 (Amer. Math. Soc., Providence, RI; Soc. Math. France, Paris, 2005).

DMZ12 A. Dabholkar, S. Murthy, and D. Zagier, Quantum black holes, wall crossing, and mock modular forms, 2012, arXiv:1208.4074.

EZ85 M. Eichler and D. Zagier, The theory of Jacobi forms, Progr. Math., vol. 55 (Birkhäuser Boston, Inc., Boston, MA, 1985); https://doi.org/10.1007/978-1-4684-9162-3. 


\section{CURVE COUNTING ON ABELIAN SURFACES AND THREEFOLDS}

FKP09 F. Flamini, A. L. Knutsen, and G. Pacienza, On families of rational curves in the Hilbert square of a surface (with an appendix by Edoardo Sernesi), Michigan Math. J. 58 (2009), no. 3, 639682; https://doi.org/10.1307/mmj/1260475694.

FP00 C. Faber and R. Pandharipande, Hodge integrals and Gromov-Witten theory, Invent. Math. 139 (2000), no. 1, 173-199; https://doi.org/10.1007/s002229900028.

FP05_L Relative maps and tautological classes, J. Eur. Math. Soc. 7 (2005), no. 1, 13-49; https://doi .org/10.4171/JEMS/20.

FP13 Tautological and non-tautological cohomology of the moduli space of curves, Handbook of Moduli, Vol. I, Adv. Lect. Math., vol. 24 (Int. Press, Somerville, MA, 2013), 293-330.

Göt90 L. Göttsche, The Betti numbers of the Hilbert scheme of points on a smooth projective surface, Math. Ann. 286 (1990), no. 1-3, 193-207; https://doi.org/10.1007/BF01453572.

Göt98_a A conjectural generating function for numbers of curves on surfaces, Comm. Math. Phys. 196 (1998), no. 3, 523-533; https://doi.org/10.1007/s002200050434.

GP99 T. Graber and R. Pandharipande, Localization of virtual classes, Invent. Math. 135 (1999), no. 2, 487-518; https://doi.org/10.1007/s002220050293.

Gra01 T. Graber, Enumerative geometry of hyperelliptic plane curves, J. Algebraic Geom. 10 (2001), no. 4, 725-755.

GS15 L. Göttsche and V. Shende, The $\mathcal{X}_{-y}$-genera of relative Hilbert schemes for linear systems on Abelian and K3 surfaces, Algebr. Geom. 2 (2015), no. 4, 405-421; https://doi.org/10. 14231/AG-2015-017.

Gul13 M.G. Gulbrandsen, Donaldson-Thomas invariants for complexes on abelian threefolds, Math. Z. 273 (2013), no. 1-2, 219-236; https://doi.org/10.1007/s00209-012-1002-3.

HM82 J. Harris and D. Mumford, On the Kodaira dimension of the moduli space of curves (with an appendix by William Fulton), Invent. Math. 67 (1982), no. 1, 23-88; https://doi.org/10. $1007 / \mathrm{BF} 01393371$.

KL13 Y.-H. Kiem and J. Li, Localizing virtual cycles by cosections, J. Amer. Math. Soc. 26 (2013), no. 4, 1025-1050; https://doi.org/10.1090/S0894-0347-2013-00768-7.

KLCM17 A. L. Knutsen, M. Lelli-Chiesa, and G. Mongardi, Severi varieties and Brill-Noether theory of curves on abelian surfaces, J. reine angew. Math., published online on 11 August 2016, https://doi.org/10.1515/crelle-2016-0029, arXiv:1503.04465, to appear in print.

KMPS10 A. Klemm, D. Maulik, R. Pandharipande, and E. Scheidegger, Noether-Lefschetz theory and the Yau-Zaslow conjecture, J. Amer. Math. Soc. 23 (2010), no. 4, 1013-1040; https://doi. org/10.1090/S0894-0347-2010-00672-8.

KT14 M. Kool and R. Thomas, Reduced classes and curve counting on surfaces I: theory, Algebr. Geom. 1 (2014), no. 3, 334-383; https://doi .org/10.14231/AG-2014-017.

KY00 T. Kawai and K. Yoshioka, String partition functions and infinite products, Adv. Theor. Math. Phys. 4 (2000), no. 2, 397-485; https://doi.org/10.4310/ATMP.2000.v4.n2.a7.

Li01 J. Li, Stable morphisms to singular schemes and relative stable morphisms, J. Differential Geom. 57 (2001), no. 3, 509-578; https://doi.org/10.4310/jdg/1090348132.

Li02 A A degeneration formula of $G W$-invariants, J. Differential Geom. 60 (2002), no. 2, 199-293; https://doi.org/10.4310/jdg/1090351102.

Loo95 E. Looijenga, On the tautological ring of $\mathscr{M}_{g}$, Invent. Math. 121 (1995), no. 2, 411-419; https: //doi.org/10.1007/BF01884306.

LS02a H. Lange and E. Sernesi, Curves of genus $g$ on an abelian variety of dimension $g$, Indag. Math. (N.S.) 13 (2002), no. 4, 523-535; https://doi.org/10.1016/S0019-3577(02) 80031-3.

LS02b - Severi varieties and branch curves of abelian surfaces of type (1,3), Internat. J. Math. 13 (2002), no. 3, 227-244; https://doi.org/10.1142/S0129167X02001381. 


\section{J. Bryan, G. Oberdieck, R. Pandharipande And Q. Yin}

MMS99 J. Maldacena, G. Moore, and A. Strominger, Counting BPS blackholes in toroidal type II string theory, 1999, arXiv:hep-th/9903163.

MNOP06 D. Maulik, N. Nekrasov, A. Okounkov, and R. Pandharipande, Gromov-Witten theory and Donaldson-Thomas theory. I, Compos. Math. 142 (2006), no. 5, 1263-1285; https://doi. org/10.1112/S0010437X06002302.

MP06 D. Maulik and R. Pandharipande, A topological view of Gromov-Witten theory, Topology 45 (2006), no. 5, 887-918; https://doi.org/10.1016/j.top.2006.06.002.

MP13_ Gromov-Witten theory and Noether-Lefschetz theory, A Celebration of Algebraic Geometry, Clay Math. Proc., vol. 18 (Amer. Math. Soc., Providence, RI, 2013), 469-507.

MPT10 D. Maulik, R. Pandharipande, and R.P. Thomas, Curves on K3 surfaces and modular forms (with an appendix by A. Pixton), J. Topol. 3 (2010), no. 4, 937-996; https://doi.org/10. 1112/jtopol/jtq030.

Obe18 G. Oberdieck, Gromov-Witten invariants of the Hilbert scheme of points of a K3 surface, Geom. Topol. 22 (2018), no. 1, 323-437.

OP16 G. Oberdieck and R. Pandharipande, Curve counting on $K 3 \times E$, the Igusa cusp form $\chi_{10}$, and descendent integration, K3 Surfaces and their Moduli, Progr. Math., vol. 315 (Birkhäuser/Springer, 2016), 245-278; https://doi.org/10.1007/978-3-319-29959-4_10.

OP06a A. Okounkov and R. Pandharipande, Gromov-Witten theory, Hurwitz theory, and completed cycles, Ann. of Math. 163 (2006), no. 2, 517-560; https://doi.org/10.4007/annals. 2006. 163.517.

OP06b_ Virasoro constraints for target curves, Invent. Math. 163 (2006), no. 1, 47-108; https: //doi.org/10.1007/s00222-005-0455-y.

OS16a G. Oberdieck and J. Shen, Curve counting on elliptic Calabi-Yau threefolds via derived categories, J. Eur. Math. Soc., to appear, arXiv:1608.07073.

OS16b_, Reduced Donaldson-Thomas invariants and the ring of dual numbers, 2016, arXiv:1612.03102.

Pir89 G. P. Pirola, Curves on generic Kummer varieties, Duke Math. J. 59 (1989), no. 3, 701-708; https://doi.org/10.1215/S0012-7094-89-05931-0.

Pon07 D. Pontoni, Quantum cohomology of $\operatorname{Hilb}^{2}\left(\mathbb{P}^{1} \times \mathbb{P}^{1}\right)$ and enumerative applications, Trans. Amer. Math. Soc. 359 (2007), no. 11, 5419-5448; https://doi.org/10.1090/ S0002-9947-07-04169-4.

PP17 R. Pandharipande and A. Pixton, Gromov-Witten/Pairs correspondence for the quintic 3-fold, J. Amer. Math. Soc. 30 (2017), no. 2, 389-449; https://doi.org/10.1090/jams/858.

PS14 B. Poonen and M. Stoll, Most odd degree hyperelliptic curves have only one rational point, Ann. of Math. 180 (2014), no. 3, 1137-1166; https://doi.org/10.4007/annals.2014.180.3.7.

PT09 R. Pandharipande and R.P. Thomas, Curve counting via stable pairs in the derived category, Invent. Math. 178 (2009), no. 2, 407-447; https://doi.org/10.1007/s00222-009-0203-9.

PT14_, 13/2 ways of counting curves, Moduli Spaces, London Math. Soc. Lecture Note Ser., vol. 411 (Cambridge Univ. Press, Cambridge, 2014), 282-333; https://doi.org/10.1017/ CB09781107279544.007.

PT16 _ The Katz-Klemm-Vafa conjecture for K3 surfaces, Forum Math. Pi 4 (2016), e4; https://doi.org/10.1017/fmp.2016.2.

Rom05 M. Romagny, Group actions on stacks and applications, Michigan Math. J. 53 (2005), no. 1, 209-236; https://doi.org/10.1307/mmj/1114021093.

Ros14 S.C.F. Rose, Counting hyperelliptic curves on an Abelian surface with quasi-modular forms, Commun. Number Theory Phys. 8 (2014), no. 2, 243-293; https://doi.org/10.4310/CNTP. 2014.v8.n2.a2. 


\section{Curve COUnting on ABElian SURFACES AND THREefoldS}

Rua06 Y. Ruan, The cohomology ring of crepant resolutions of orbifolds, Gromov-Witten Theory of Spin Curves and Orbifolds, Contemp. Math., vol. 403 (Amer. Math. Soc., Providence, RI, 2006), 117-126; https://doi.org/10.1090/conm/403/07597.

She15 J. Shen, The Euler characteristics of generalized Kummer schemes, Math. Z. 281 (2015), no. 3, 1183-1189; https://doi.org/10.1007/s00209-015-1526-4.

STV15 T. Schürg, B. Toën, and G. Vezzosi, Derived algebraic geometry, determinants of perfect complexes, and applications to obstruction theories for maps and complexes, J. reine angew. Math. 702 (2015), 1-40; https://doi.org/10.1515/crelle-2013-0037.

Tat65 J. T. Tate, Algebraic cycles and poles of zeta functions, Arithmetical Algebraic Geometry (Proc. Conf. Purdue Univ., 1963) (Harper \& Row, New York, 1965), 93-110.

Wis11 J. Wise, The genus zero Gromov-Witten invariants of the symmetric square of the plane, Comm. Anal. Geom. 19 (2011), no. 5, 923-974; https://doi.org/10.4310/CAG.2011.v19. n5.a5.

Wis16 , A hyperelliptic Hodge integral, Port. Math. 73 (2016), no. 3, 207-218; https://doi. org/10.4171/PM/1985.

YZ96 S.-T. Yau and E. Zaslow, BPS states, string duality, and nodal curves on K3, Nuclear Phys. B 471 (1996), no. 3, 503-512; https://doi.org/10.1016/0550-3213(96)00176-9.

Jim Bryan jbryan@math.ubc.ca

Department of Mathematics, The University of British Columbia, 1984 Mathematics Road, Vancouver, BC, V6T 1Z2, Canada

Georg Oberdieck georgo@mit.edu

Department of Mathematics, Massachusetts Institute of Technology, 77 Massachusetts Ave., Cambridge, MA 02139, USA

Rahul Pandharipande rahul@math.ethz.ch

Department of Mathematics, ETH Zürich, Rämistrasse 101, HG G 33.4, 8092 Zürich, Switzerland

Qizheng Yin yinqizheng@math.pku.edu.cn

Beijing International Center for Mathematical Research, Peking University, 5 Yiheyuan Road, Haidian District, 100871 Beijing, China 